ANDRÉ AMARO BUENO

Esparsificação de dicionários para métodos kernel 
ANDRÉ AMARO BUENO

\title{
Esparsificação de dicionários para métodos kernel
}

\author{
VERSÃO CORRIGIDA
}

Dissertação apresentada à Escola Politécnica da

Universidade de São Paulo para obtenção do título de Mestre em Ciências. 


\title{
Esparsificação de dicionários para métodos kernel
}

\author{
VERSÃO CORRIGIDA
}

Dissertação apresentada à Escola Politécnica da Universidade de São Paulo para obtenção do título de Mestre em Ciências.

Área de concentração:

Engenharia de Sistemas Eletrônicos

Orientador:

Prof. Dr. Magno Teófilo Madeira da Silva 
Autorizo a reprodução e divulgação total ou parcial deste trabalho, por qualquer meio convencional ou eletrônico, para fins de estudo e pesquisa, desde que citada a fonte.

Este exemplar foi revisado e corrigido em relação à versão original, sob responsabilidade única do autor e com a anuência de seu orientador.

São Paulo, 08 de setembro de 2020.

$$
\text { culul Chunf trewn }
$$

André Amaro Bueno

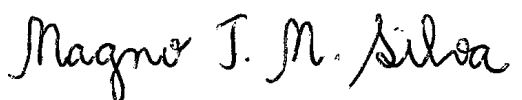

Prof. Dr. Magno Teófilo Madeira da Silva

\section{Catalogação-na-publicação}

Bueno, André Amaro

Esparsificação de dicionários para métodos kernel/ A. A. Bueno, M. T. M. Silva -- São Paulo, 2020.

79 p.

Dissertação (Mestrado) - Escola Politécnica da Universidade de São Paulo. Departamento de Engenharia de Sistemas Eletrônicos.

1. Processamento de sinais adaptativos 2. Filtros elétricos adaptativos I.Universidade de São Paulo. Escola Politécnica. Departamento de Engenharia de Sistemas Eletrônicos II.t 
Aos meus pais,

Luís Alberto e Maria Emilia 


\section{AGRADECIMENTOS}

Agradeço ao meu orientador, Prof. Dr. Magno Teófilo Madeira da Silva, pelos inúmeros conselhos, pela receptividade, atenção e tempo dedicado à orientação da pesquisa que levou a este trabalho.

Aos meus pais, Luís Alberto e Maria Emilia, por estarem sempre presentes e por terem me proporcionado tudo o que puderam ao longo de toda a vida.

Ao meu irmão Eduardo, pelas conversas, companheirismo e trocas, sempre necessárias.

Aos professores José Carlos Bermudez e Aline Neves Panazio pelas contribuições e sugestões.

À Coordenação de Aperfeiçoamento de Pessoal de Nível Superior (CAPES), pelo financiamento. 


\section{RESUMO}

BUENO, André Amaro. Esparsificação de dicionários para métodos kernel / A. A. Bueno. Dissertação (Mestrado) apresentada à Escola Politécnica da Universidade de São Paulo. 76f. São Paulo, 2020

Métodos baseados em núcleo (kernel) são capazes de resolver problemas não lineares, projetando o vetor de entrada em um espaço de dimensão mais alta, onde se utiliza um método linear. Esses métodos têm sido amplamente utilizados em conjunto com diferentes técnicas de processamento de sinais e aprendizado de máquina, como máquinas de vetores de suporte, análise de componentes principais, filtragem adaptativa, entre outras. Uma das maiores dificuldades encontradas nesses métodos é a necessidade de armazenar um conjunto de vetores de entrada, denominado dicionário. Muitas vezes, o dicionário pode se tornar grande demais o que causa um aumento exagerado no custo computacional. Para evitar um crescimento muito grande do dicionário, surgiram na literatura diferentes técnicas de esparsificação. Neste trabalho, é proposta uma técnica de esparsificação de dicionários para métodos kernel baseada no processo de ortogonalização de Gram-Schmidt. Ela projeta os vetores mapeados pelo kernel em um subespaço de dimensão finita gerado por uma base ortonormal e pode ser usada com qualquer kernel de Mercer. Em particular, a técnica proposta é aplicada a um algoritmo do tipo LMS (least-mean-square). Resultados de simulação mostram que o algoritmo proposto consegue manter um bom desempenho em termos de erro quadrático médio e apresenta um custo computacional menor quando comparado com o algoritmo kernel LMS implementado com outras técnicas de esparsificação. Por fim, também se propõe uma forma de retirar vetores do dicionário, o que é particularmente interessante quando há alterações no ambiente em que o algoritmo é empregado.

Palavras-chave: métodos baseados em kernel, esparsificação de dicionários, filtragem adaptativa, processamento de sinais não-lineares 


\begin{abstract}
BUENO, André Amaro. Dictionary sparsification for kernel methods / A. A. Bueno. Dissertation (Masters) presented to Escola Politécnica da Universidade de São Paulo. 76f. São Paulo, 2020

Kernel based methods are capable of solving nonlinear problems by projecting the input vectors in a higher dimensional space where a linear method is used. These methods have been widely used in conjunction with different techniques of signal processing and machine learning, as support vector machines, principal component analysis, adaptive filtering among others. One of the main drawbacks of these methods is that a set of input vectors, known as dictionary, needs to be stored in memory. In certain situations, the dictionary may become too big, which leads to a high computational cost. To avoid an exagerated growth of the dictionary, different sparsification techniques were proposed in the literature. In this work, we propose a sparsification technique for kernel methods based on the Gram-Schmidt orthogonalization process. It projects the vectors mapped by the kernel into a finite-dimensional subspace spanned by an orthonormal basis and can be used with any Mercer kernel. In particular, the proposed technique is applied to a least-mean-square-type (LMS) algorithm. Simulation results show that the proposed algorithm mantains a good mean-square-error performance and presents a smaller computational cost, when compared with the kernel LMS algorithm using other sparsification techniques. Furthermore, we also propose a method for removing vectors from the dictionary, which is particularly interesting when there are changes the environment where the algorithm is used.
\end{abstract}

Keywords: kernel-based-methods, dictionary sparsification, adaptive filtering, nonlinear signal processing. 


\section{SUMÁRIO}

1 Introdução e Formulação do Problema 1

1.1 Justificativa e objetivos $\ldots \ldots \ldots \ldots \ldots \ldots$

1.2 Contribuições do trabalho . . . . . . . . . . . . . . . . . 8

1.3 Estrutura da dissertação . . . . . . . . . . . . . . . . . . . . . . . 9

2 Revisitando técnicas de esparsificação $\quad 10$

2.1 Novelty Criterion $(\mathrm{NC}) \ldots \ldots \ldots \ldots \ldots$

2.2 Coherence Criterion $(\mathrm{CC}) \ldots \ldots \ldots \ldots$

2.3 Vector Quantization $(\mathrm{VQ}) \ldots \ldots \ldots \ldots \ldots$. . . . . . . . . . . 11

2.4 Random Fourier Features $(\mathrm{RFF}) \quad \ldots \ldots \ldots$

2.5 Approximate Linear Dependency (ALD) . . . . . . . . . . . . . . . . . . . . . 15

2.6 Resultados de simulação . . . . . . . . . . . . . . . . . . . . . . 17

2.7 Conclusão . . . . . . . . . . . . . . . . . . . . . . . . . . . . . 19

3 Ortogonalização de Gram-Schmidt para esparsificação de dicionários 23

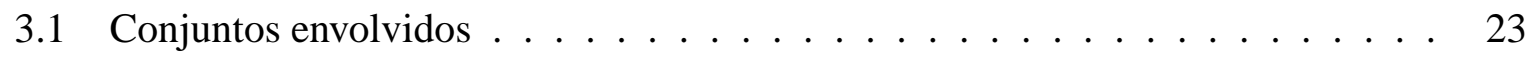

3.2 Representando os vetores mapeados como projeções de dimensão finita . . . . 25

3.3 Forma matricial do processo de Gram-Schmidt . . . . . . . . . . . . . . 26

3.4 Relações entre as matrizes $\mathbf{H}_{i}$ e $\mathbf{G}_{i} \ldots \ldots \ldots$. . . . . . . . . . . 29

3.5 Selecionando vetores para a base . . . . . . . . . . . . . . 30

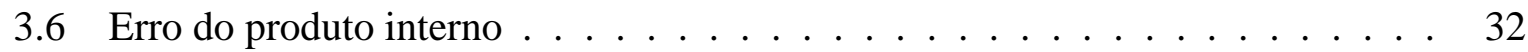

3.7 Resultados de simulação: pré-imagem . . . . . . . . . . . . . . . . . 33

3.8 Conclusão . . . . . . . . . . . . . . . . . . . . . . . . . . . 34 
4.1 Aplicando a esparsificação proposta no KLMS: o algoritmo Gram-Schmidt KLMS 37

4.1.1 Resultados de Simulação . . . . . . . . . . . . . . . . . . 39

4.1.1.1 Predição não-linear com kernel gaussiano . . . . . . . . . . 39

4.1.1.2 Predição não-linear com kernel polinomial . . . . . . . . . . 44

4.1.1.3 Predição de série temporal de manchas solares . . . . . . . . 46

4.1.1.4 Equalização de canal não linear . . . . . . . . . . . . . . . . 49

4.1.1.5 Análise do MSE variando-se o $\varepsilon_{G S}$ para kernel gaussiano . . 53

4.1.1.6 Análise do MSE variando-se o $\varepsilon_{G S}$ para kernel polinomial . . 56

4.2 Retirando elementos do dicionário . . . . . . . . . . . . . . . . . 58

4.2.1 Resultados de Simulação . . . . . . . . . . . . . . . . . . . 60

4.3 Conclusão . . . . . . . . . . . . . . . . . . . . . . . 61

5 Conclusões $\quad 66$

$\begin{array}{ll}\text { Referências } & 68\end{array}$ 


\section{INTRODUÇÃO E FORMULAÇÃO DO PROBLEMA}

A solução de problemas não lineares é de interesse na engenharia. Sistemas dinâmicos podem ter características não lineares e séries temporais associadas a esses sistemas também as tem (ALLIGOOD; SAUER; YORKE, 1997). Dessa forma, é preciso considerar não linearidades nas soluções de muitos problemas envolvendo identificação de sistemas, predição de séries temporais, classificação, regressão, equalização de canais de comunicação, entre outros (LIU; PRÍNCIPE; HAYKIN, 2010; BISHOP, 2006; MURPHY, 2012). A literatura contém diferentes técnicas não lineares. Por exemplo, nos problemas de classificação e regressão, utilizam-se em geral as redes neurais e as máquinas de vetores de suporte (support vector machines - SVM) (GOODFELLOW; BENGIO; COURVILLE, 2016; VAPNIK, 1995). Esses métodos precisam de exemplos para serem treinados e testados antes de serem utilizados de fato, ou seja, seu aprendizado não é dado em tempo real. Nos problemas de predição de séries temporais, identificação de sistemas e equalização de canais em que se deseja um aprendizado em tempo real, soluções baseadas em filtros adaptativos são mais adequadas (COMMINIELLO; PRINCIPE, 2018; LIU; PRÍNCIPE; HAYKIN, 2010). Nesse contexto, surgem os filtros adaptativos baseados em núcleo (kernel) que têm despertado interesse na literatura como uma boa alternativa entre as soluções não lineares (COMMINIELLO; PRINCIPE, 2018).

Métodos baseados em núcleo (kernel) são capazes de resolver problemas não lineares, projetando o vetor de entrada em um espaço de dimensão mais alta, onde um método linear é usado (LIU; PRÍNCIPE; HAYKIN, 2010). Para ilustrar, considere o problema de classificação entre os pontos azuis e laranjas da Figura 1-1. Na Figura 1-2, é possível observar que é necessário uma solução não linear no espaço $\mathbb{R}^{2}$ para resolver o problema. No caso, com uma circunferência no $\mathbb{R}^{2}$, é uma possível classificar os pontos corretamente. Projetando os pontos para um espaço de dimensão maior, no caso o $\mathbb{R}^{3}$, como mostrado na Figura 1-3, pode-se usar uma solução linear (um plano) como mostrado na Figura 1-4. Com esse exemplo simples, é possível visualizar que problemas não lineares podem ser resolvidos de maneira linear em espaços de dimensão mais alta, que é o fundamento dos métodos baseados em kernel.

Esses métodos têm sido amplamente utilizados em conjunto com diferentes técnicas de processamento de sinais e aprendizado de máquina, como SVM, análise de componentes principais (principal componente analysis - PCA), filtragem adaptativa, entre outras 

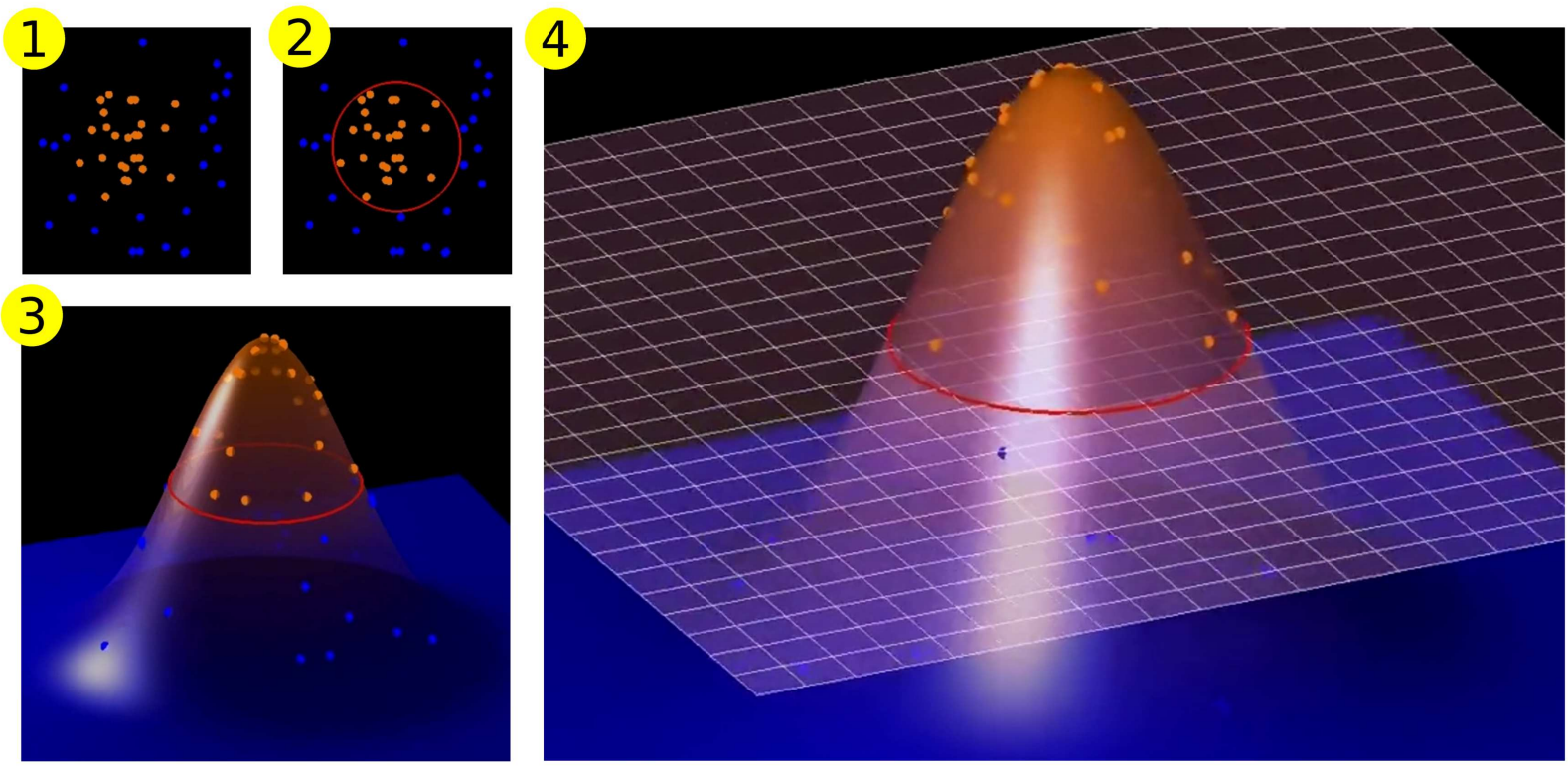

Figura 1: Ilustração de um problema de classificação não linear no espaço $\mathbb{R}^{2}$, que pode ser resolvido de maneira linear no espaço $\mathbb{R}^{3}$.

Fonte: (TEAMGRIZZLY, 2010)

(SCHOLKOPF; SMOLA, 2002; LIU; PRÍNCIPE; HAYKIN, 2010). Apesar dessas técnicas terem objetivos diferentes, todas elas mapeiam um vetor coluna $\mathbf{u} \in \mathcal{U} \subset \mathbb{R}^{M}$, sendo $M$ a dimensão do espaço de entrada, em um espaço de alta dimensão $\mathcal{H}$ como $\varphi(\mathbf{u})$, usando um kernel de Mercer (SCHOLKOPF; SMOLA, 2002; LIU; POKHAREL; PRÍNCIPE, 2008; LIU; PRÍNCIPE; HAYKIN, 2010). É importante salientar que nesta dissertação só serão tratados sinais cujo domínio é o conjunto dos reais.

Kernel de Mercer é uma função contínua, simétrica e positiva-definida $\kappa: \mathcal{U} \times \mathcal{U} \rightarrow \mathbb{R}$, tal que para todo $\mathbf{u}, \mathbf{v} \in \mathcal{U}$ vale (LIU; PRÍNCIPE; HAYKIN, 2010)

$$
\kappa(\mathbf{u}, \mathbf{v})=\langle\varphi(\mathbf{u}), \varphi(\mathbf{v})\rangle_{\mathcal{H}} \triangleq \varphi(\mathbf{u})^{T} \varphi(\mathbf{v})
$$

em que o superescrito $T$ representa transposição. Essa equação é conhecida como truque do kernel já que o produto interno dos vetores mapeados $\varphi(\mathbf{u})$ e $\varphi(\mathbf{v})$ pode ser calculado de maneira eficiente no espaço $\mathcal{H}$, sem se conhecer explicitamente a função $\varphi(\cdot)$ (LIU; PRÍNCIPE; HAYKIN, 2010). O kernel gaussiano é o mais utilizado na literatura e é definido como

$$
\kappa(\mathbf{u}, \mathbf{v})=e^{-\zeta\|\mathbf{u}-\mathbf{v}\|^{2}},
$$

em que $\|\cdot\|$ denota a norma euclidiana, $\zeta=1 /\left(2 \sigma^{2}\right)$ é o parâmetro do kernel e $\sigma$ sua largura. 
Pelo teorema de Mercer, o kernel gaussiano induz um espaço de Hilbert $\mathcal{H}$ de dimensão infinita (LIU; PRÍNCIPE; HAYKIN, 2010). Outro kernel bastante utilizado na literatura é o kernel polinomial, dado por

$$
\kappa(\mathbf{u}, \mathbf{v})=(\langle\mathbf{u}, \mathbf{v}\rangle+1)^{q},
$$

em que $q$ é um inteiro maior que um, chamado de parâmetro do kernel. Este kernel, por sua vez, induz um espaço de Hilbert $\mathcal{H}$ de dimensão finita. Esses não são os únicos kernels de Mercer possíveis, porém, esta dissertação mantém o foco nesses dois kernels por serem os mais usados na literatura. Os resultados aqui apresentados podem ser estendidos diretamente para outros kernels de Mercer.

Graças ao truque da Equação (1.1), técnicas que utilizam produtos internos, como é o caso de filtros adaptativos, podem ter versões baseadas em kernel. Dentre os diferentes filtros adaptativos kernel da literatura, o algoritmo kernel least-mean-squares (KLMS) (LIU; POKHAREL; PRÍNCIPE, 2008) é o mais popular graças à sua simplicidade. No espaço de alta dimensão $\mathcal{H}$, o algoritmo LMS é usado para atualizar o vetor coluna dos coeficientes no espaço induzido de características, denotado por $\omega(n-1)$, a fim de se estimar o sinal desejado $d(n)$. As equações do KLMS são dadas por

$$
\begin{aligned}
y(n) & =\varphi(\mathbf{u}(n))^{T} \omega(n-1), \\
e(n) & =d(n)-y(n), \\
\omega(n) & =\omega(n-1)+\mu e(n) \varphi(\mathbf{u}(n)),
\end{aligned}
$$

em que $\omega(0)=\mathbf{0}, \mathbf{u}(n)$ é o vetor de entrada do filtro, $y(n)$ é a sua saída, $e(n)$ representa o erro de estimação e $\mu$ um passo de adaptação. Um diagrama de blocos mostrando a aplicação do KLMS em um problema de identificação de sistemas é apresentado na Figura 2. Nessa figura, $v(n)$ é um ruído de medição.

Pelo Teorema da Representação, utilizando o truque do kernel de (1.1) e o fato de que $\boldsymbol{\omega}(0)=\mathbf{0}, \boldsymbol{\omega}(n-1)$ pode ser expresso como uma combinação não linear das amostras do passado de modo que a saída do filtro pode ser obtida como (LIU; POKHAREL; PRÍNCIPE, 2008)

$$
y(n)=\varphi(\mathbf{u}(n))^{T} \omega(n-1)=\mu \sum_{i=1}^{n-1} e(i) \kappa(\mathbf{u}(n), \mathbf{u}(i)) .
$$

O cálculo de $y(n)$ utiliza os vetores de entrada do filtro desde o instante inicial até o instante $n-1$, ou seja, os vetores do conjunto $\mathcal{D}(n)=\left\{\mathbf{u}_{1}=\mathbf{u}(1), \mathbf{u}_{2}=\mathbf{u}(2), \cdots, \mathbf{u}_{n-1}=\mathbf{u}(n-1)\right\}$. Esse conjunto é denominado dicionário e sua cardinalidade é $N(n)=n-1$. Calcular a saída como 


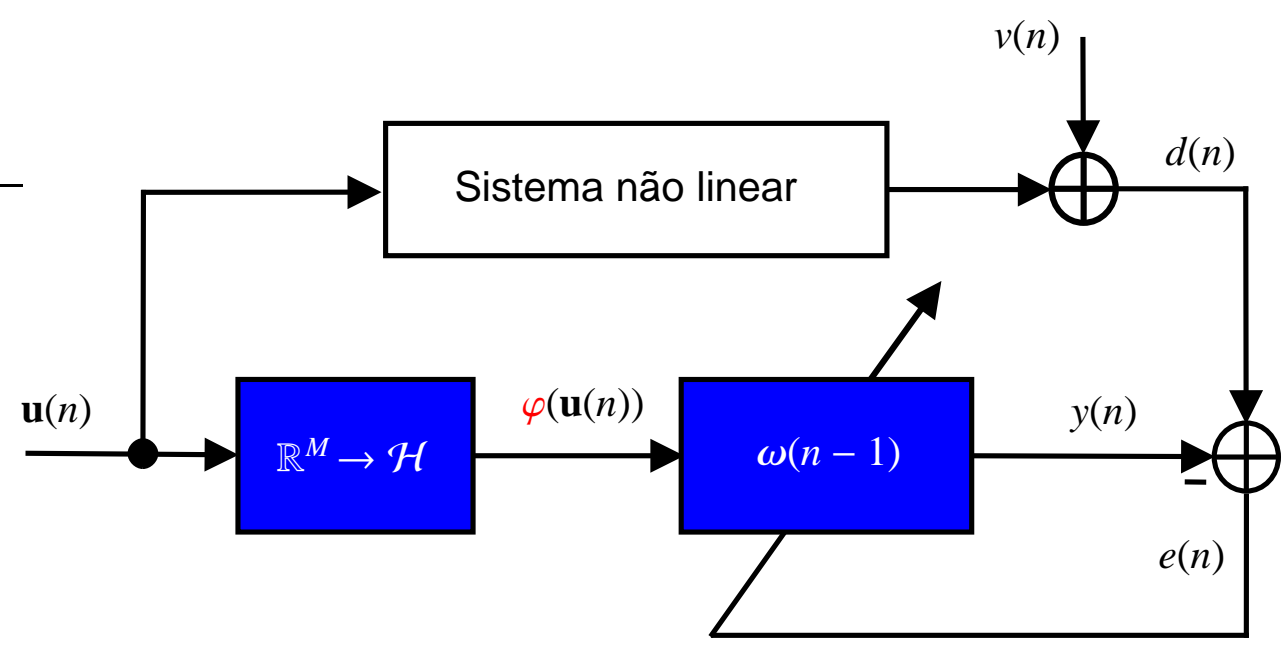

Figura 2: Aplicação do KLMS em um problema de identificação de sistemas.

Fonte: Autor

(1.7) é particularmente interessante quando o kernel é gaussiano. Nesse caso, as dimensões de $\varphi(\mathbf{u}(n))$ e $\omega(n)$ são infinitas, o que impossibilita o cálculo como em (1.4). As Equações (1.5) e (1.7) constituem o algoritmo KLMS. Como é necessário guardar $\mu e(n)$ em cada iteração, é comum definir o parâmetro $a_{n}=\mu e(n)$ referente ao vetor $\mathbf{u}(n)$ do dicionário. As equações do KLMS são mostradas na Tabela 1, em que alguns índices foram suprimidos para simplificar a notação.

Tabela 1: Sumário do algoritmo KLMS.

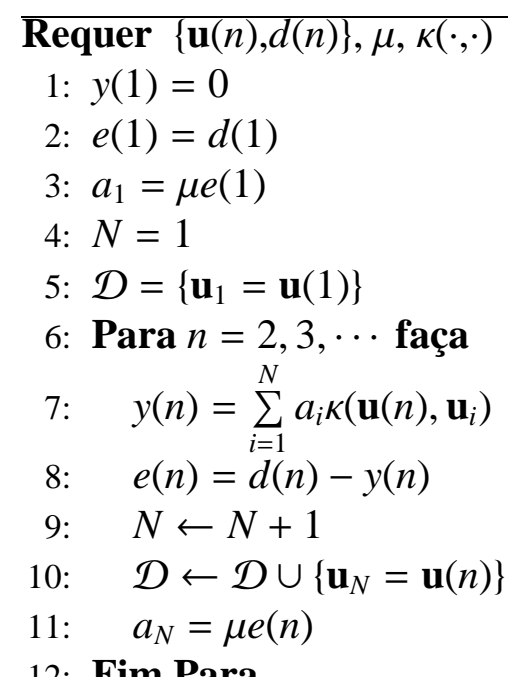

Fonte: Autor

De (1.7), observa-se que um dos maiores problemas dos métodos baseados em kernel é o elevado custo computacional já que o dicionário cresce linearmente com o tempo. Diante disso, algumas técnicas de esparsificação foram propostas na literatura (ver, e.g., (RICHARD; BERMUDEZ; HONEINE, 2009; LIU; PRÍNCIPE; HAYKIN, 2010; 
ENGEL; MANNOR; MEIR, 2004) e suas referências). Essas técnicas proporcionam uma redução do custo computacional de métodos baseados em kernel e são importantes para possibilitar o uso deses métodos em problemas que exigem soluções em tempo real.

Para ilustrar o comportamento do KLMS, considerou-se a seguir a predição da série temporal dada por (YUKAWA, 2012; SILVA et al., 2018)

$$
\begin{aligned}
& d(n)=\left[0,8-0,5 \exp \left(-d^{2}(n-1)\right)\right] d(n-1) \\
& -\left[0,3+0,9 \exp \left(-d^{2}(n-1)\right)\right] d(n-2)+0,1 \sin (d(n-1) \pi),
\end{aligned}
$$

em que $d(n)=0,1$ para $n<0$. Em $n=1,5 \times 10^{4}$, considerou-se uma mudança abrupta levando à seguinte sequência

$$
\begin{aligned}
& d(n)=\left[0,2-0,7 \exp \left(-d^{2}(n-1)\right)\right] d(n-1) \\
& -\left[0,8+0,8 \exp \left(-d^{2}(n-1)\right)\right] d(n-2)+0,2 \sin (d(n-1) \pi) .
\end{aligned}
$$

Nesse cenário, é possível verificar uma nova convergência do algoritmo a partir de $n=1,5 \times 10^{4}$. Consideraram-se ainda o kernel gaussiano com parâmetro do kernel $\zeta=1$, vetores de entrada de dimensão $M=7$, ou seja,

$$
\mathbf{u}(n)=[u(n) u(n-1) \cdots u(n-6)]^{T}
$$

e o horizonte de predição de uma amostra. Assim, $u(n)=d(n-1)$. O passo de adaptação escolhido foi $\mu=0,5$. Na comparação, considerou-se o erro quadrático médio (mean square error - MSE), definido como $\mathrm{E}\left[e^{2}(n)\right]$, em que $\mathrm{E}[\cdot]$ representa a esperança matemática que é estimada a partir de uma média de conjunto das realizações. Como neste exemplo não se considerou ruído, a média de conjunto é dada por uma única realização.

O gráfico do MSE obtido é mostrado na Figura 3, assim como o desempenho do LMS convencional. É possível observar o bom desempenho do KLMS obtendo baixos níveis de erro e conseguindo convergir após a mudança abrupta na série temporal, diferente do LMS que não obteve um bom resultado, principalmente após a mudança abrupta. O tamanho do dicionário está na Figura 4, e como era esperado ele cresce linearmente ao longo das iterações. O número de operações de multiplicação e operações não lineares acumuladas estão nas Figuras 5 e 6 respectivamente. Como o LMS convencional não utiliza um dicionário e nem funções não lineares, ele não foi considerado nas Figuras 4 e 6 . Os resultados dessa simulação mostram a importância 


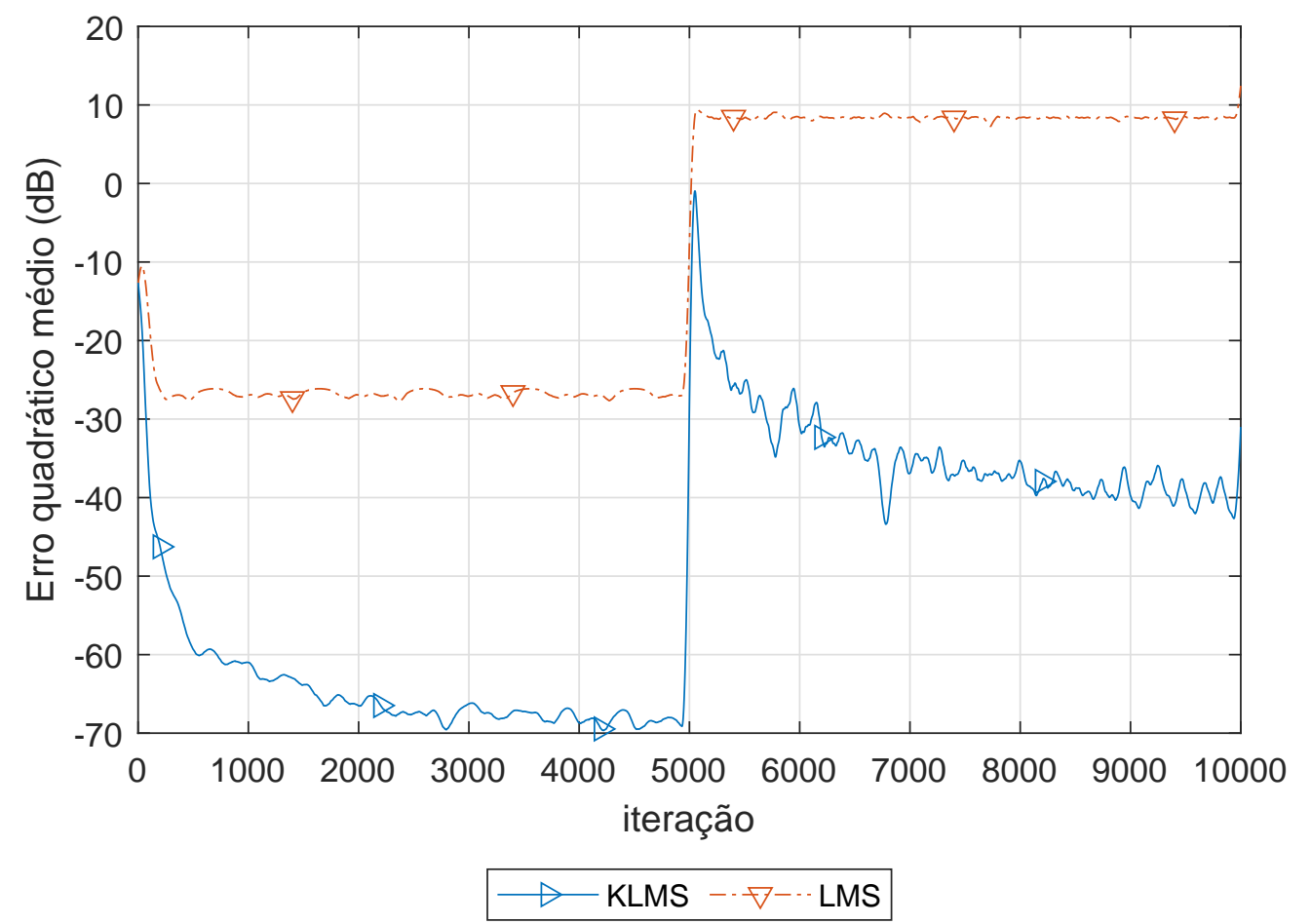

Figura 3: MSE para o problema de predição não linear considerando kernel gaussiano, $\zeta=1$, $M=7$ e $\mu=0,5$.

Fonte: Autor.

de se usar uma solução não linear neste problema. Apesar de um custo computacional muito maior, o KLMS apresenta um MSE mais de $40 \mathrm{~dB}$ menor que o do LMS na predição das duas séries temporais. O maior problema do KLMS é o número de operações acumuladas devido ao tamanho do dicionário, que por sua vez cresce linearmente ao longo das iterações. Isso mostra a importância das técnicas de esparsificação de dicionário.

\subsection{Justificativa e objetivos}

Atualmente, o custo computacional dos métodos baseados em kernel ainda os tornam proibitivos em problemas que necessitam de soluções em tempo real. Técnicas de esparsificação têm surgido como uma forma de diminuir o custo computacional por meio da redução do dicionário. Diante disso, o estudo de técnicas que proporcionem uma redução ainda maior no custo computacional sem causar uma degradação exagerada no desempenho dos métodos baseados em kernel é algo de interesse na comunidade de processamento de sinais e aprendizagem de máquina.

Os objetivos desta dissertação são: 


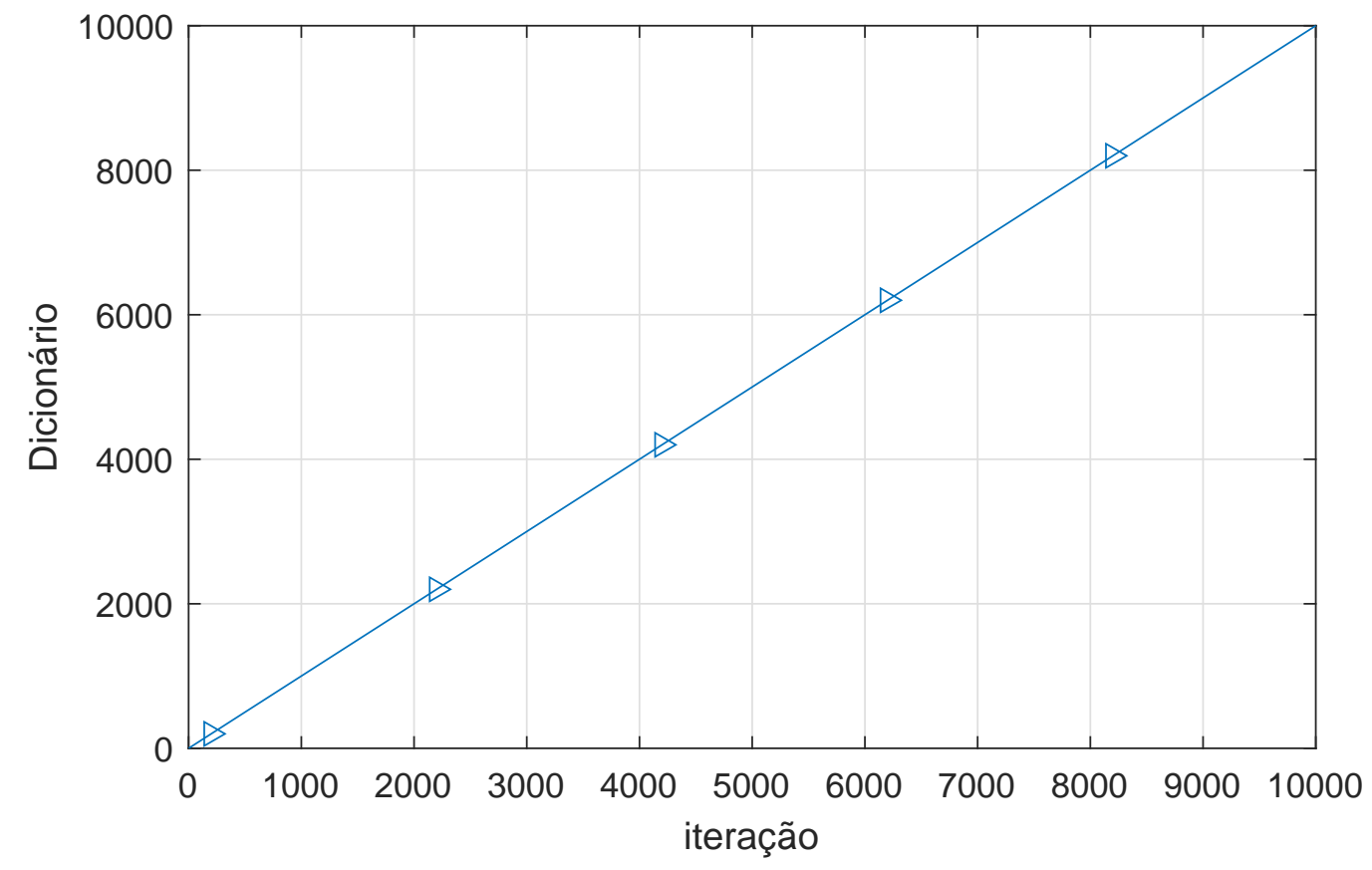

$\triangleright-$ KLMS

Figura 4: Tamanho do dicionário para o problema de predição não linear considerando kernel gaussiano, $\zeta=1, M=7$ e $\mu=0,5$.

Fonte: Autor.

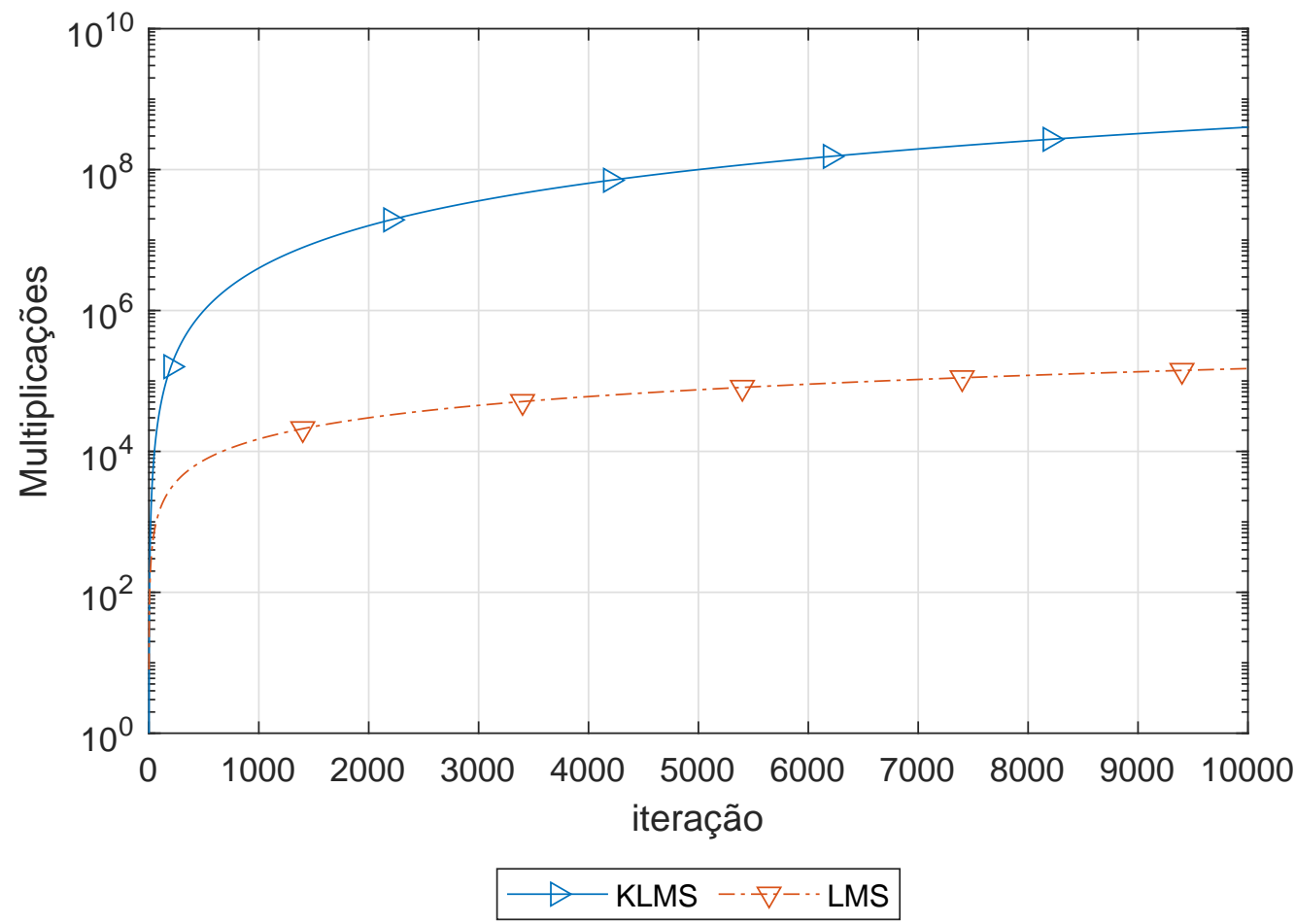

Figura 5: Número de operações de multiplicação acumuladas para o problema de predição não linear considerando kernel gaussiano, $\zeta=1, M=7$ e $\mu=0,5$.

Fonte: Autor. 


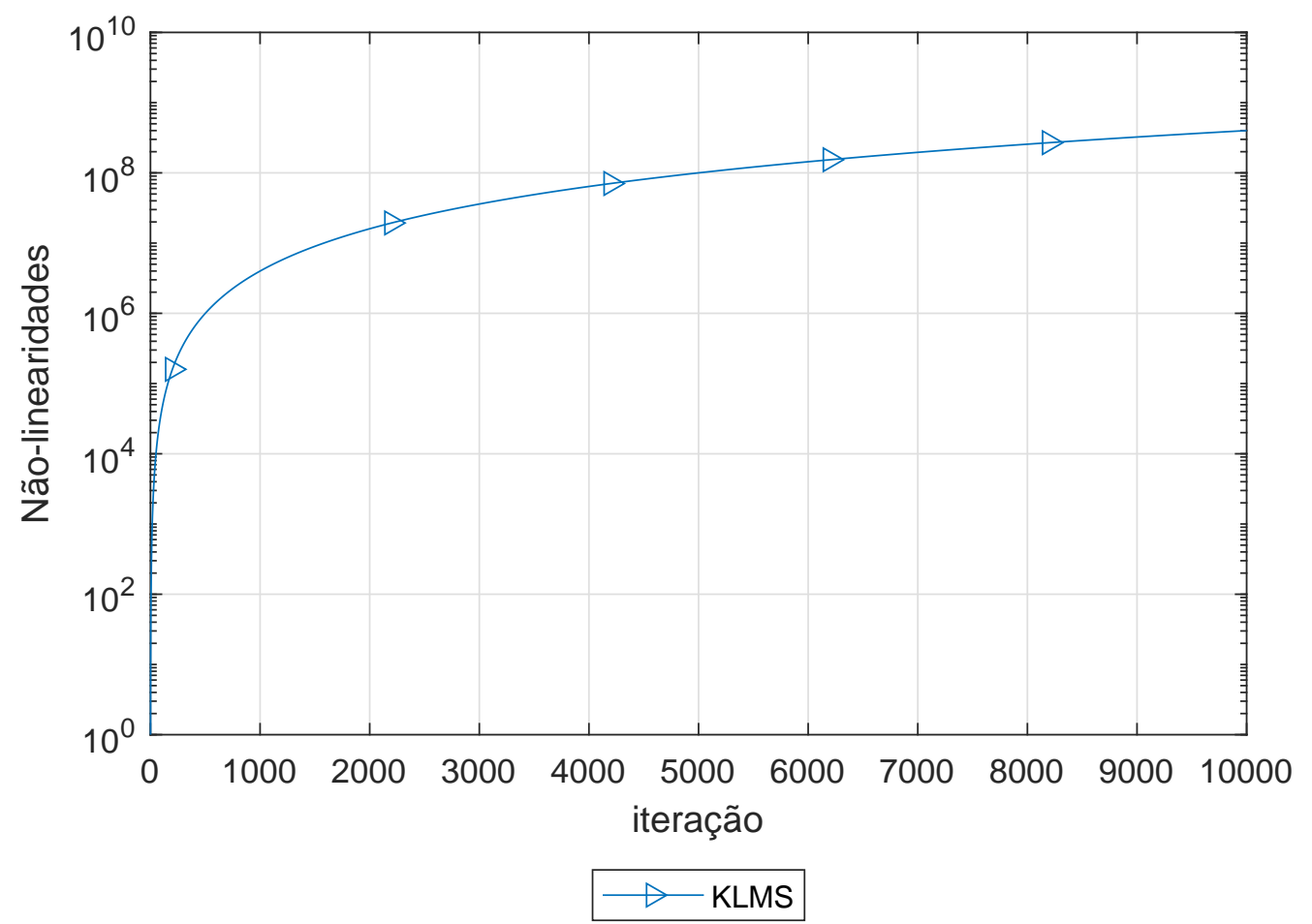

Figura 6: Número de operações não lineares acumuladas para o problema de predição não linear considerando kernel gaussiano, $\zeta=1, M=7$ e $\mu=0,5$.

Fonte: Autor.

- propor uma nova técnica de esparsificação que reduza o custo computacional sem degradar muito o desempenho de métodos baseados em kernel;

- aplicar essa técnica a um algoritmo de filtragem adaptativa do tipo KLMS;

- propor uma forma para retirar elementos do dicionário que não tenham muita influência no resultado.

\subsection{Contribuições do trabalho}

Este trabalho apresenta as seguintes contribuições:

- proposta de uma técnica de esparsificação de dicionários baseada no processo de GramSchimidt;

- aplicação da técnica proposta ao algoritmo KLMS;

- proposta de um método para retirar elementos do dicionário. 
Parte dessas contribuições foi publicada nos anais de um simpósio nacional e submetida a uma revista internacional, como especificado a seguir.

- André A. Bueno, Magno T. M. Silva: "Esparsificação de dicionário para métodos kernel baseada na ortogonalização de Gram-Schmidt”, Anais do Simpósio Brasileiro de Telecomunicações e Processamento de Sinais (SBrT), Petrópolis, 29 de setembro a 2 de outubro de 2019, doi:10.14209/SBRT.2019.1570556819.

- André A. Bueno, Magno T. M. Silva: “Gram-Schmidt-based sparsification for kernel dictionary”, IEEE Signal Processing Letters, vol. 27, p. 1130-1134, 2020.

\subsection{Estrutura da dissertação}

A presente dissertação começa expondo no Capítulo 2 técnicas de esparsificação de dicionários encontradas na literatura. Cinco técnicas e suas aplicações ao algoritmo KLMS são descritas. Uma maior ênfase é dada à técnica da dependência linear aproximada devido à sua semelhança com a técnica proposta nesta dissertação. É necessário deixar claro que a aplicação dessa técnica ao algoritmo KLMS não é encontrada na literatura, mas foi estendida aqui para efeitos de comparação.

O Capítulo 3 descreve a técnica de esparsificação proposta, partindo do processo de ortogonalização de Gram-Schmidt e obtendo as equações necessárias para se calcular as projeções dos vetores em um espaço de dimensão finita. Também é apresentado um estudo sobre o limite do erro no produto interno quando a técnica proposta é utilizada na esparsificação de métodos baseados em kernel.

O Capítulo 4 aplica a técnica proposta ao KLMS e compara o desempenho e custo computacional do algoritmo resultante com as outras técnicas apresentadas anteriormente. Além disso, uma forma de retirar vetores do dicionário é proposta.

As conclusões e sugestões para trabalhos futuros são apresentadas no Capítulo 5. 


\section{REVISITANDO TÉCNICAS DE ESPARSIFICAÇÃO}

Neste capítulo, cinco técnicas de esparsificação utilizadas em conjunto com o algoritmo KLMS são revisitadas. Essas técnicas são usadas na comparação com a técnica de esparsificação proposta. Cabe observar que na maior parte delas, a Equação (1.7) da página 3 se reduz para

$$
y(n)=\mu \sum_{i=1}^{N(n)} e(i) \kappa\left(\mathbf{u}(n), \mathbf{u}_{i}\right),
$$

já que a cardinalidade $N(n)$ do dicionário $\mathcal{D}(n)$ não concide mais $\operatorname{com} n-1$, pelo fato de apenas alguns vetores de entrada serem parte do dicionário.

\subsection{Novelty Criterion (NC)}

O critério da novidade (Novelty Criterion, NC), proposto em (LIU; PRÍNCIPE; HAYKIN, 2010), decide se o vetor $\mathbf{u}(n)$ deve ou não fazer parte do dicionário, calculando a distância entre esse vetor e todos os vetores já contidos no dicionário, $\mathbf{u}_{i}, i=1,2, \cdots, N(n)$. Se a distância mínima for maior que o limiar positivo $\varepsilon_{N C}$, ou seja, se

$$
\min _{i}\left\|\mathbf{u}(n)-\mathbf{u}_{i}\right\|>\varepsilon_{N C}
$$

o vetor $\mathbf{u}(n)$ é incluído no dicionário como o elemento $\mathbf{u}_{N(n+1)} \operatorname{com} N(n+1)=N(n)+1$. Caso contrário, o dicionário e sua cardinalidade permanecem como antes, ou seja, $\mathcal{D}(n+1)=\mathcal{D}(n)$ e $N(n+1)=N(n)$. Quando a norma é menor que o limiar, conjectura-se que o vetor $\mathbf{u}(n)$ não traz nova informação já que existe um vetor no dicionário que está a uma distância relativamente pequena dele. A aplicação desse método ao KLMS é dada na Tabela 2.

\subsection{Coherence Criterion (CC)}

O critério da coerência (Coherence Criterion, $\mathrm{CC}$ ), proposto em (RICHARD; BERMUDEZ; HONEINE, 2009), decide se o vetor de entrada u( $n)$ deve ou não fazer parte do dicionário, calculando o produto interno entre o vetor $\mathbf{u}(n)$ e todos os vetores do dicionário $\mathbf{u}_{i}, i=1,2, \cdots, N(n)$ no espaço induzido pelo kernel. Utilizando o truque do kernel (1.1), deve-se comparar o maior valor absoluto entre os produtos internos citados com o 
Tabela 2: Sumário do algoritmo NC-KLMS.

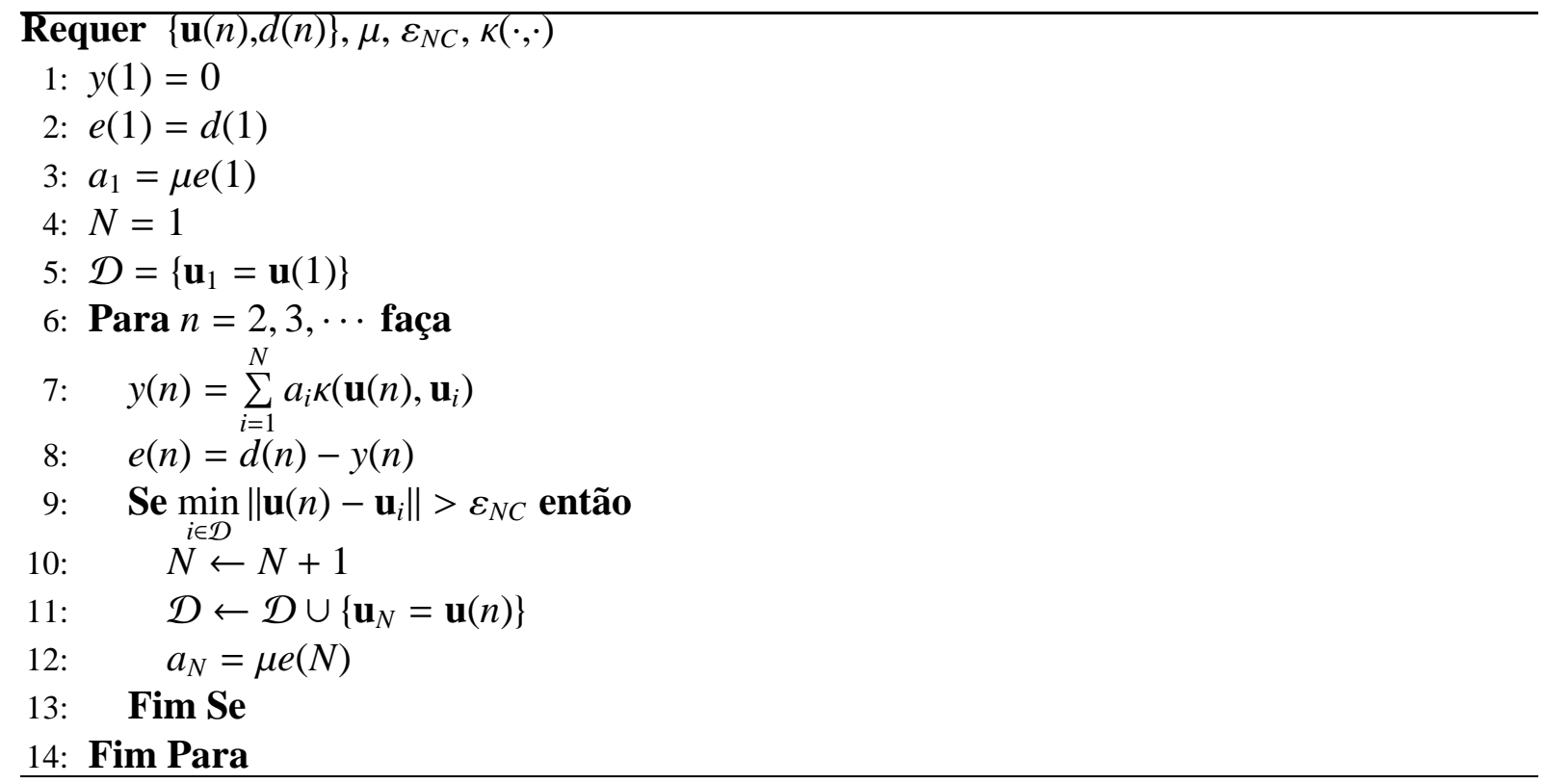

Fonte: Autor.

limiar positivo $\varepsilon_{C C}$ e, caso seja menor, ou seja, caso

$$
\max _{i}\left|\kappa\left(\mathbf{u}(n), \mathbf{u}_{i}\right)\right| \leq \varepsilon_{C C}
$$

o vetor $\mathbf{u}(n)$ é incluído no dicionário como o elemento $\mathbf{u}_{N(n+1)}$ com $N(n+1)=N(n)+1$. Caso contrário, o dicionário e sua cardinalidade permanecem como antes, ou seja, $\mathcal{D}(n+1)=\mathcal{D}(n)$ e $N(n+1)=N(n)$. O cálculo do produto interno no espaço induzido pelo kernel mostra uma similaridade entre o vetor $\mathbf{u}(n)$ e os vetores mapeados. A decisão de incluir ou não $\mathbf{u}(n)$ no dicionário é tomada baseada em quão similar esse vetor é em relação aos demais vetores contidos no dicionário. No caso do kernel gaussiano, o valor do produto interno no espaço induzido pelo kernel está relacionado à distância euclidiana e portanto, este critério é equivalente ao da novidade. A aplicação desse método ao KLMS é dada na Tabela 3.

\subsection{Vector Quantization (VQ)}

Quantização vetorial (Vector Quantization, VQ) é uma operação que aproxima um vetor por outro de forma que o conjunto de vetores possíveis de serem armazenados no dicionário seja menor. Um exemplo de quantização vetorial é o critério da novidade que utiliza a distância no espaço de entrada para verificar que um vetor de entrada é próximo o suficiente de um outro que já está no dicionário. Com base nessa aproximação, o NC decide se o vetor de entrada deve 
Tabela 3: Sumário do algoritmo CC-KLMS.

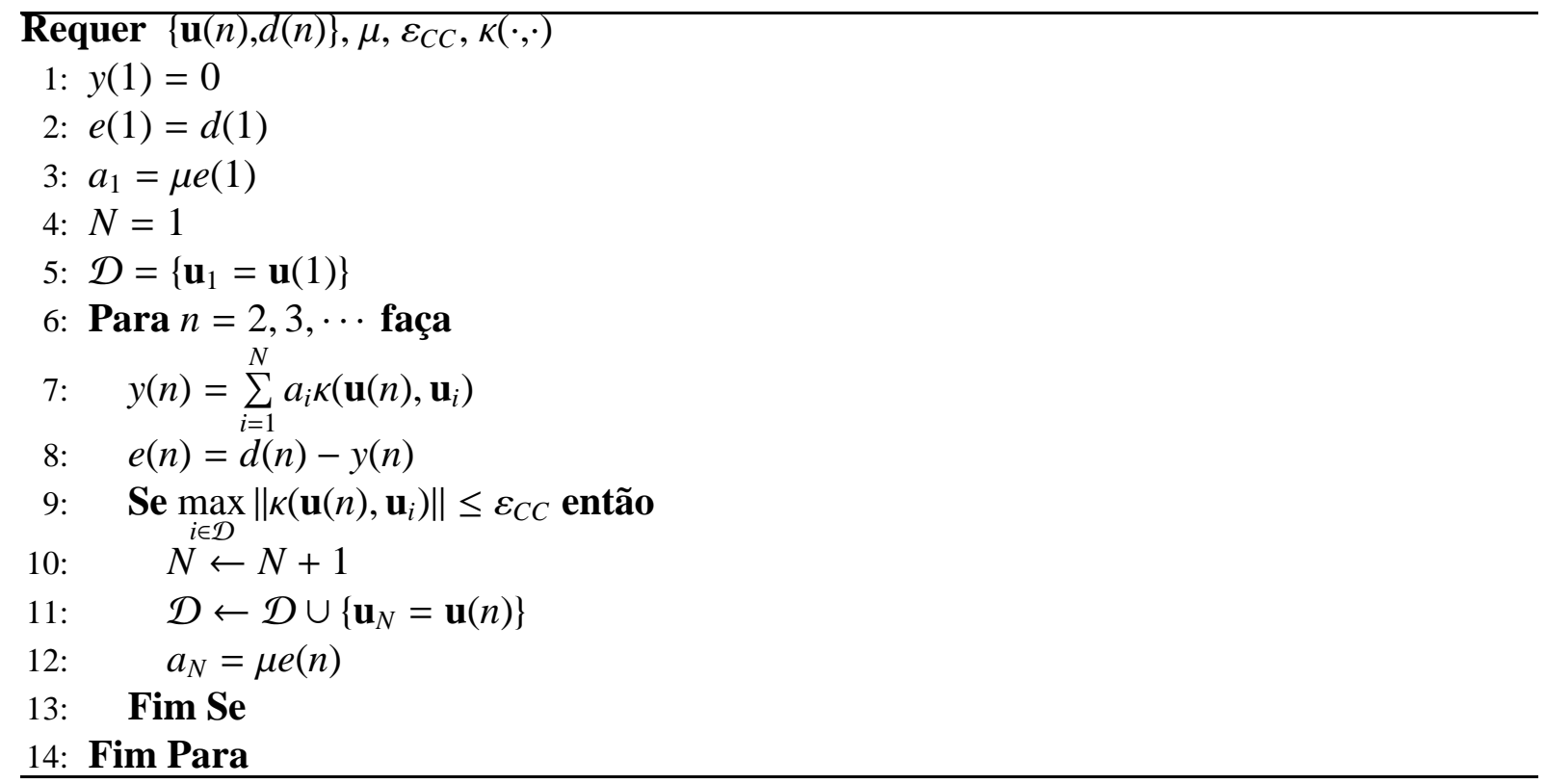

Fonte: Autor.

ou não ser inserido no dicionário. Em (CHEN et al., 2012a), é proposto utilizar a quantização vetorial do critério da novidade, o que levou ao algoritmo KLMS quantizado (quantized KLMS - QKLMS). Nesse algoritmo, quando o vetor de entrada $\mathbf{u}(n)$ não é incluído no dicionário pelo critério da novidade, o valor de $a_{i}$ relativo ao vetor do dicionário mais próximo de $\mathbf{u}(n)$ é adaptado como

$$
a_{i}=a_{i}+\mu e(n)
$$

em que $e(n)=d(n)-y(n)$ e $y(n)$ calculado como em (2.1). O uso de dados redundantes para adaptar o coeficiente do vetor mais próximo leva a um algoritmo com melhor acurácia e um dicionário mais compacto em comparação com o NC aplicado ao KLMS. O QKLMS é descrito na Tabela 4.

\subsection{Random Fourier Features (RFF)}

O método das características aleatórias de Fourier (Random Fourier Features, RFF), proposto em (SINGH; AHUJA; MOULIN, 2012; BOUBOULIS; POUGKAKIOTIS; THEODORIDIS, 2016), se baseia no teorema de Bochner para gerar um algoritmo que não tem dicionário, aproximando o cálculo do kernel por um produto interno de vetores de características. Com isso, é possível chegar a um algoritmo cujo custo computacional é fixo. Considerando-se um kernel invariante à translação 
Tabela 4: Sumário do algoritmo QKLMS.

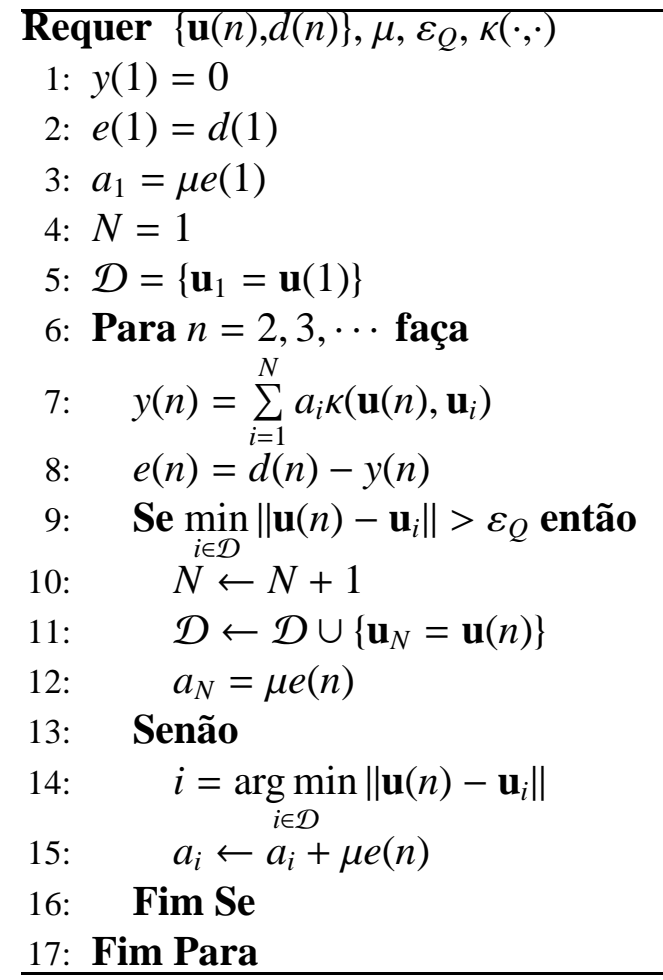

Fonte: Autor.

$\kappa(\mathbf{u}, \mathbf{v})=\kappa_{I}(\mathbf{v}-\mathbf{u})=\kappa_{I}(\gamma)$, segundo o teorema de Bochner (RAHIMI; RECHT, 2008), sua transformada de Fourier

$$
p(\boldsymbol{\Omega})=\frac{1}{(2 \pi)^{M}} \int_{\mathbb{R}^{M}} \kappa_{I}(\boldsymbol{\gamma}) e^{-i \mathbf{\Omega}^{T} \gamma} d \boldsymbol{\gamma}
$$

pode ser entendida como uma função densidade de probabilidade em que $\boldsymbol{\Omega}$ é um vetor de frequências. É possível, portanto, calcular o valor do kernel como a transformada inversa de Fourier

$$
\kappa(\mathbf{u}, \mathbf{v})=\kappa_{I}(\boldsymbol{\gamma})=\int_{\mathbb{R}^{M}} p(\mathbf{\Omega}) e^{i \mathbf{\Omega}^{T} \gamma} d \mathbf{\Omega} .
$$

Como esse valor é real, pode-se calcular essa transformada inversa como

$$
\kappa(\mathbf{u}, \mathbf{v})=\int_{\mathbb{R}^{M}} p(\mathbf{\Omega}) \cos \left(\mathbf{\Omega}^{T}(\mathbf{v}-\mathbf{u})\right) d \mathbf{\Omega} .
$$

Somando uma parcela nula que será útil mais adiante tem-se

$$
\kappa(\mathbf{u}, \mathbf{v})=\int_{\mathbb{R}^{M}} p(\boldsymbol{\Omega}) \cos \left(\boldsymbol{\Omega}^{T}(\mathbf{v}-\mathbf{u})\right) d \boldsymbol{\Omega}+\int_{\mathbb{R}^{M}} \int_{0}^{2 \pi} p(\mathbf{\Omega}) \cos \left(\mathbf{\Omega}^{T}(\mathbf{v}+\mathbf{u})+\theta\right) d \theta d \boldsymbol{\Omega},
$$


em que $\theta$ é uma variável aleatória uniformemente distribuída no intervalo $[0,2 \pi]$. Colocando $p(\mathbf{\Omega})$ em evidência, chega-se a

$$
\kappa(\mathbf{u}, \mathbf{v})=\int_{\mathbb{R}^{M}} \int_{0}^{2 \pi} p(\mathbf{\Omega})\left(\cos \left(\mathbf{\Omega}^{T}(\mathbf{v}-\mathbf{u})\right)+\cos \left(\mathbf{\Omega}^{T}(\mathbf{v}+\mathbf{u})+\theta\right)\right) d \theta d \mathbf{\Omega} .
$$

Utilizando uma identidade trigonométrica, obtém-se

$$
\left.\kappa(\mathbf{u}, \mathbf{v})=\int_{\mathbb{R}^{M}} \int_{0}^{2 \pi} p(\mathbf{\Omega}) 2 \cos \left(\mathbf{\Omega}^{T} \mathbf{v}+\theta\right) \cos \left(\boldsymbol{\Omega}^{T} \mathbf{u}+\theta\right)\right) d \theta d \mathbf{\Omega} .
$$

Identificando-se essa equação como o cálculo da esperança sobre as variáveis aleatórias $\Omega$ e $\theta$, chega-se a

$$
\left.\kappa(\mathbf{u}, \mathbf{v})=E_{\mathbf{\Omega}, t}\left[2 \cos \left(\mathbf{\Omega}^{T} \mathbf{v}+t\right) \cos \left(\mathbf{\Omega}^{T} \mathbf{u}+\theta\right)\right)\right] .
$$

Definindo

$$
z_{\boldsymbol{\Omega}, \theta}(\mathbf{u}) \triangleq \sqrt{2} \cos \left(\mathbf{\Omega}^{T} \mathbf{u}+\theta\right)
$$

tem-se que

$$
\kappa(\mathbf{u}, \mathbf{v})=E_{\boldsymbol{\Omega}, \theta}\left[z_{\boldsymbol{\Omega}, \theta}(\mathbf{u})^{T} z_{\boldsymbol{\Omega}, \theta}(\mathbf{v})\right] .
$$

Dessa forma, a esperança pode ser aproximada por uma média aritmética e é possível montar o vetor

$$
\mathbf{z}(\mathbf{u})=\sqrt{\frac{2}{D}}\left[\begin{array}{c}
\cos \left(\boldsymbol{\Omega}_{1}^{T} \mathbf{u}+\theta_{1}\right) \\
\cos \left(\boldsymbol{\Omega}_{2}^{T} \mathbf{u}+\theta_{2}\right) \\
\vdots \\
\cos \left(\mathbf{\Omega}_{D}^{T} \mathbf{u}+\theta_{D}\right)
\end{array}\right]
$$

tal que $\kappa(\mathbf{u}, \mathbf{v}) \approx \mathbf{z}(\mathbf{u})^{T} \mathbf{z}(\mathbf{v})$. Assim, é possível aplicar esses vetores ao algoritmo KLMS. Para o caso do kernel gaussiano, a função densidade de probabilidade associada é a normal e os valores $\theta_{i}, i=1,2, \cdots, D$ têm uma densidade uniforme no intervalo $[0,2 \pi]$. A aplicação desta técnica ao KLMS utilizando o kernel gaussiano é mostrada na Tabela 5. Cabe observar que não há uma versão desse algoritmo para o kernel polinomial, pois este não é invariante à translação. 
Tabela 5: Sumário do algoritmo RFF-KLMS.

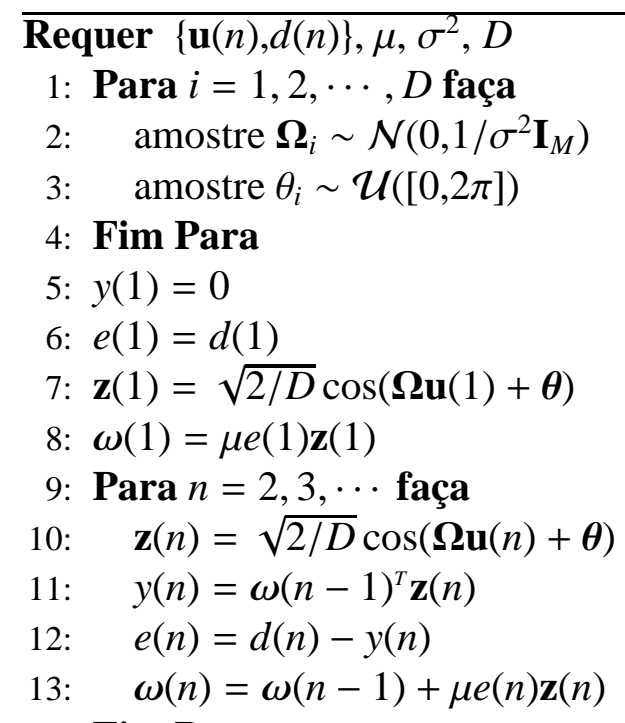

14: Fim Para

Fonte: Autor.

\subsection{Approximate Linear Dependency (ALD)}

O algoritmo da dependência linear aproximada (Approximate Linear Dependency, ALD) utiliza o seguinte critério para selecionar vetores para o dicionário:

$$
\min _{\mathbf{b}(n)}\left\|\sum_{i=1}^{N(n)} b_{i}(n) \varphi\left(\mathbf{u}_{i}\right)-\varphi(\mathbf{u}(n))\right\|^{2}>\varepsilon_{A L D}
$$

em que $\mathbf{b}(n)=\left[b_{1}(n) b_{2}(n) \cdots b_{N(n)}(n)\right]$ é um vetor de coeficientes de dimensão finita e $\varepsilon_{A L D} \mathrm{O}$ limiar de comparação. A ideia dessa técnica é substituir $\varphi(\mathbf{u}(n))$ por $\mathbf{b}(n)$ nas Equações (1.4) e (1.6). O vetor $\mathbf{b}(n)$ é composto pelos coeficientes da combinação linear dos vetores mapeados $\varphi\left(\mathbf{u}_{1}\right), \varphi\left(\mathbf{u}_{2}\right), \cdots, \varphi\left(\mathbf{u}_{N(n)}\right)$, que criam a melhor aproximação do vetor mapeado $\varphi(\mathbf{u}(n))$. Para se obter o vetor $\mathbf{b}(n)$, deve-se desenvolver a norma que aparece em (2.15) e utilizar as propriedades lineares do produto interno, o que leva a

$$
\sum_{i=1}^{N(n)} \sum_{j=1}^{N(n)} b_{i}(n) b_{j}(n)\left\langle\varphi\left(\mathbf{u}_{i}\right), \boldsymbol{\varphi}\left(\mathbf{u}_{j}\right)\right\rangle-2 \sum_{i=1}^{N(n)} b_{i}(n)\left\langle\varphi\left(\mathbf{u}_{i}\right), \boldsymbol{\varphi}(\mathbf{u}(n))\right\rangle+\langle\boldsymbol{\varphi}(\mathbf{u}(n)), \boldsymbol{\varphi}(\mathbf{u}(n))\rangle>\varepsilon_{A L D}
$$

Definindo

$$
\boldsymbol{\kappa}_{N(n)}\left(\mathbf{u}_{\ell}\right) \triangleq\left[\begin{array}{llll}
\kappa\left(\mathbf{u}_{\ell}, \mathbf{u}_{1}\right) & \kappa\left(\mathbf{u}_{\ell}, \mathbf{u}_{2}\right) & \cdots & \kappa\left(\mathbf{u}_{\ell}, \mathbf{u}_{N(n)}\right)
\end{array}\right]^{T},
$$

para um $\mathbf{u}_{\ell}$ qualquer, a desigualdade (2.16) pode ser reescrita usando o gramiano $\mathbf{G}_{N(n)}$, que é uma matriz simétrica em que cada elemento é dado pelo produto interno entre dois vetores, ou seja, o elemento da linha $i$ e coluna $j$ dessa matriz é $g_{i j}=\left\langle\varphi\left(\mathbf{u}_{i}\right), \varphi\left(\mathbf{u}_{j}\right)\right\rangle$. O subscrito $N(n)$ indica 
a dimensão da matriz. No caso, levando em conta os produtos internos de todos os vetores do dicionário mapeados, essa matriz tem dimensão $N(n) \times N(n)$ e pode ser calculada como

$$
\mathbf{G}_{N(n)}=\left[\begin{array}{llll}
\boldsymbol{\kappa}_{N(n)}\left(\mathbf{u}_{1}\right) & \boldsymbol{\kappa}_{N(n)}\left(\mathbf{u}_{2}\right) & \cdots & \boldsymbol{\kappa}_{N(n)}\left(\mathbf{u}_{N(n)}\right)
\end{array}\right] .
$$

É possível provar que o gramiano é uma matriz positiva semi-definida e que seu determinante é zero apenas se os vetores que foram usados nos produtos internos formem um conjunto linearmente dependente (SCHOLKOPF; SMOLA, 2002). Utilizando (2.17) e (2.18), pode-se reescrever (2.16) como

$$
\mathbf{b}^{T}(n) \mathbf{G}_{N(n)} \mathbf{b}(n)-2 \mathbf{b}^{T}(n) \boldsymbol{\kappa}_{N(n)}(\mathbf{u}(n))+\kappa(\mathbf{u}(n), \mathbf{u}(n))>\varepsilon_{A L D} .
$$

Derivando (2.19) em relação ao vetor $\mathbf{b}(n)$ e igualando ao vetor nulo para se obter o mínimo, chega-se a

$$
2 \mathbf{G}_{N(n)} \mathbf{b}(n)-2 \boldsymbol{\kappa}_{N(n)}(\mathbf{u}(n))=\mathbf{0} .
$$

A partir de (2.20), obtém-se

$$
\mathbf{b}(n)=\mathbf{G}_{N(n)}^{-1} \boldsymbol{\kappa}_{N(n)}(\mathbf{u}(n))
$$

Substituindo este resultado em (2.19), chega-se a

$$
\kappa(\mathbf{u}(n), \mathbf{u}(n))-\boldsymbol{\kappa}_{N(n)}(\mathbf{u}(n))^{T} \mathbf{G}_{N(n)}^{-1} \boldsymbol{\kappa}_{N(n)}(\mathbf{u}(n))>\varepsilon_{A L D}
$$

Para se calcular o produto interno de dois vetores $\varphi(\mathbf{u}(n))$ e $\varphi(\mathbf{u}(m))$ a partir de suas aproximações $\mathbf{b}(n)$ e $\mathbf{b}(m)$, é necessário utilizar o gramiano, pois

$$
\begin{aligned}
\langle\boldsymbol{\varphi}(\mathbf{u}(n)), \boldsymbol{\varphi}(\mathbf{u}(m))\rangle & \approx\left\langle\sum_{i=1}^{N(n)} b_{i}(n) \boldsymbol{\varphi}\left(\mathbf{u}_{i}\right), \sum_{j=1}^{N(n)} b_{j}(m) \boldsymbol{\varphi}\left(\mathbf{u}_{j}\right)\right\rangle \\
& \approx \sum_{i=1}^{N(n)} \sum_{j=1}^{N(n)} b_{i}(n) b_{j}(m)\left\langle\boldsymbol{\varphi}\left(\mathbf{u}_{i}\right), \boldsymbol{\varphi}\left(\mathbf{u}_{j}\right)\right\rangle,
\end{aligned}
$$

o que leva a

$$
\langle\varphi(\mathbf{u}(n)), \varphi(\mathbf{u}(m))\rangle \approx \mathbf{b}(n)^{T} \mathbf{G}_{N(n)} \mathbf{b}(m) .
$$

A aproximação nessa expressão é dada por conta do critério (2.15) já que o vetor b(n) contém os coeficientes da aproximação de $\varphi(u(n))$. É possível notar que a desigualdade (2.22) é equivalente a

$$
\|\varphi(\mathbf{u}(n))\|^{2}-\|\mathbf{b}(n)\|^{2}>\varepsilon_{A L D}
$$


Em (ENGEL; MANNOR; MEIR, 2004), não é dada nenhuma interpretação geométrica para esse critério. Uma equação equivalente a essa será apresentada no Capítulo 3 e sua interpretação geométrica será dada. Cabe observar que o vetor $\mathbf{b}(n)$ é uma representação do vetor $\varphi(\mathbf{u}(n))$ em uma base não ortogonal formada por $\varphi\left(\mathbf{u}_{1}\right), \varphi\left(\mathbf{u}_{2}\right), \cdots, \varphi\left(\mathbf{u}_{N(n)}\right)$.

Para se calcular a representação $\mathbf{b}(n)$, o ALD usa a matriz inversa do gramiano como mostrado em (2.21). As matrizes $\mathbf{G}_{N(n)}$ e sua inversa devem ser atualizadas sempre que um vetor for incluído no dicionário. Para isso, (ENGEL; MANNOR; MEIR, 2004) sugere as formas iterativas

$$
\mathbf{G}_{N(n)+1}=\left[\begin{array}{cc}
\mathbf{G}_{N(n)} & \boldsymbol{\kappa}_{N(n)}(\mathbf{u}(n)) \\
\boldsymbol{\kappa}_{N(n)}(\mathbf{u}(n))^{T} & \kappa(\mathbf{u}(n), \mathbf{u}(n))
\end{array}\right],
$$

e

$$
\mathbf{G}_{N(n)+1}^{-1}=\frac{1}{\delta}\left[\begin{array}{cc}
\left(\delta \mathbf{G}_{N(n)}^{-1}+\mathbf{b}(n) \mathbf{b}(n)^{T}\right) & -\mathbf{b}(n) \\
-\mathbf{b}(n)^{T} & 1
\end{array}\right],
$$

em que $\delta=\kappa(\mathbf{u}(n), \mathbf{u}(n))-\boldsymbol{\kappa}_{N(n)}(\mathbf{u}(n))^{T} \mathbf{b}(n)$. A aplicação desse método ao algoritmo KLMS é dado na Tabela 6.

\subsection{Resultados de simulação}

Tomando-se novamente o exemplo da Seção 1 da página 5, as técnicas descritas neste capítulo são comparadas com o KLMS sem esparsificação em termos de MSE, tamanho do dicionário e operações acumuladas. Todos os filtros utilizam o mesmo parâmetro do kernel e passo de adaptação daquela simulação, ou seja, $\zeta=1$ e $\mu=0,5$. Além disso, a dimensão dos vetores de entrada é $M=7$ e o horizonte de predição é uma amostra. Os limiares das técnicas foram ajustados de modo a obter um bom resultado em termos de MSE em comparação com o KLMS sem esparsificação. Além disso, para verificar alguma diferença entre o QKLMS e o NC-KLMS, foi escolhido o mesmo limiar para ambos, dessa forma ambos terão o mesmo tamanho de dicionário mas espera-se um melhor desempenho do QKLMS. Dessa forma, foram escolhidos $\varepsilon_{N C}=\varepsilon_{Q}=0,01$ e $\varepsilon_{C C}=e^{-\varepsilon_{N C}^{2}}=0,9999$. O limiar escolhido para o ALD-KLMS foi $\varepsilon_{A L D}=0,0199$ e a dimensão do vetor de características aleatórias do RFF foi $D=5000$.

Pode-se notar na Figura 7 que todos os filtros obtêm resultados próximos ao do KLMS sem esparsificação em termos de MSE. Porém, o QKLMS tem alguma vantagem em relação ao NC-KLMS e CC-KLMS. Além disso, o RFF-KLMS tem um desempenho pior, chegando a uma diferença de aproximadamente $10 \mathrm{~dB}$, quando comparado aos outros na primeira metade 
Tabela 6: Sumário do algoritmo ALD-KLMS.

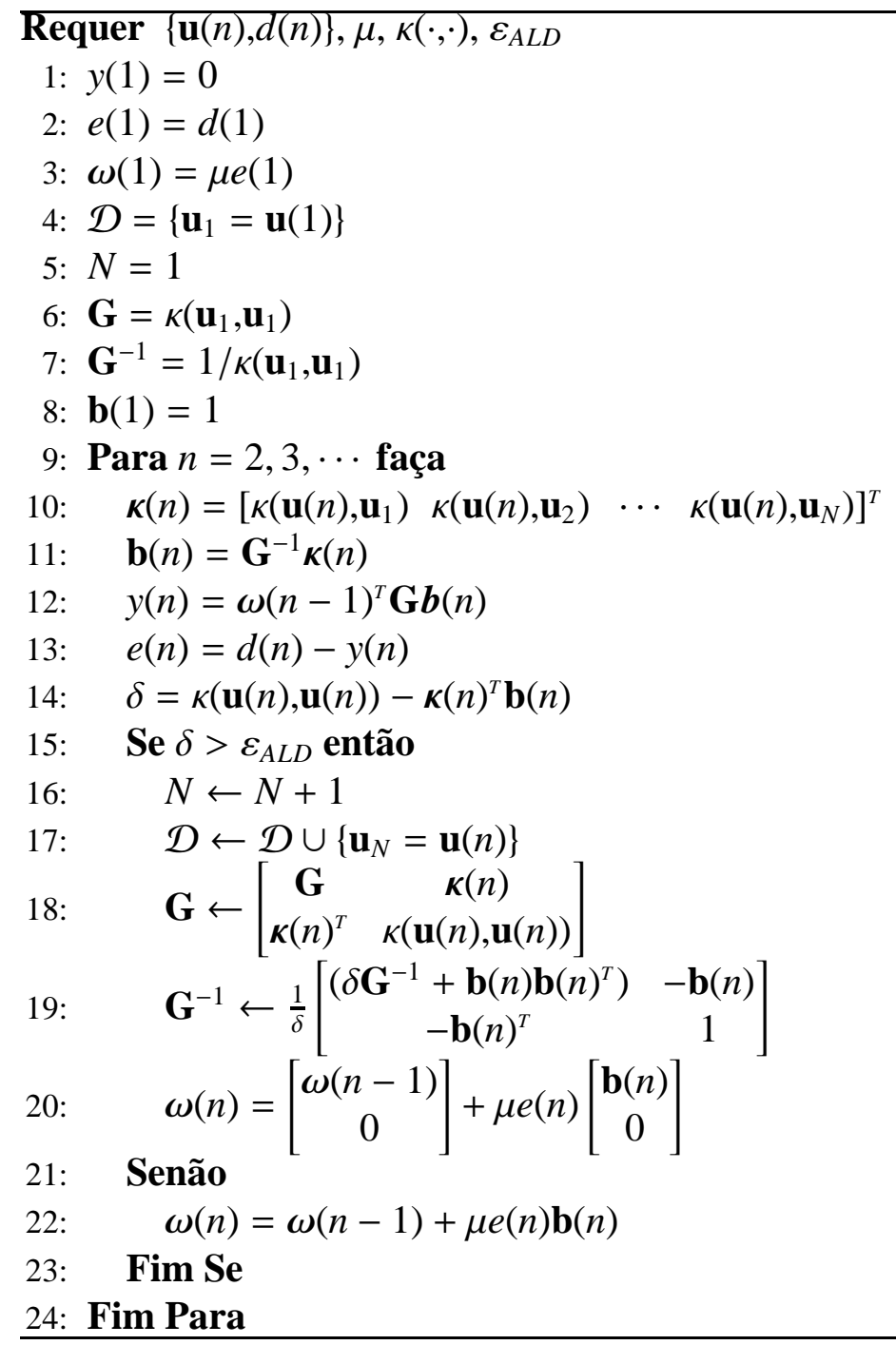

Fonte: Autor.

do experimento. Seria possível melhorar o desempenho do RFF-KLMS nessa primeira metade, aumentando o tamanho do vetor de características. No entanto, seu custo computacional aumentaria muito e ele já apresenta um erro quadrático médio similar ao dos demais na segunda metade do experimento.

Na Figura 8, são mostrados os tamanhos dos dicionários dos filtros. Não é mostrado o tamanho do dicionário do RFF-KLMS, pois ele não considera explicitamente um dicionário. Sendo assim, verifica-se que a diminuição do tamanho do dicionário em relação ao KLMS sem esparsificação é significativa e que o ALD-KLMS consegue um desempenho comparável com os outros algoritmos mesmo com um dicionário menor. O QKLMS possui um dicionário de mesmo tamanho que o NC-KLMS como esperado. O número de operações de multiplicação e de operações não lineares acumuladas estão respectivamente nas Figuras 9 e 10, mostrando que 


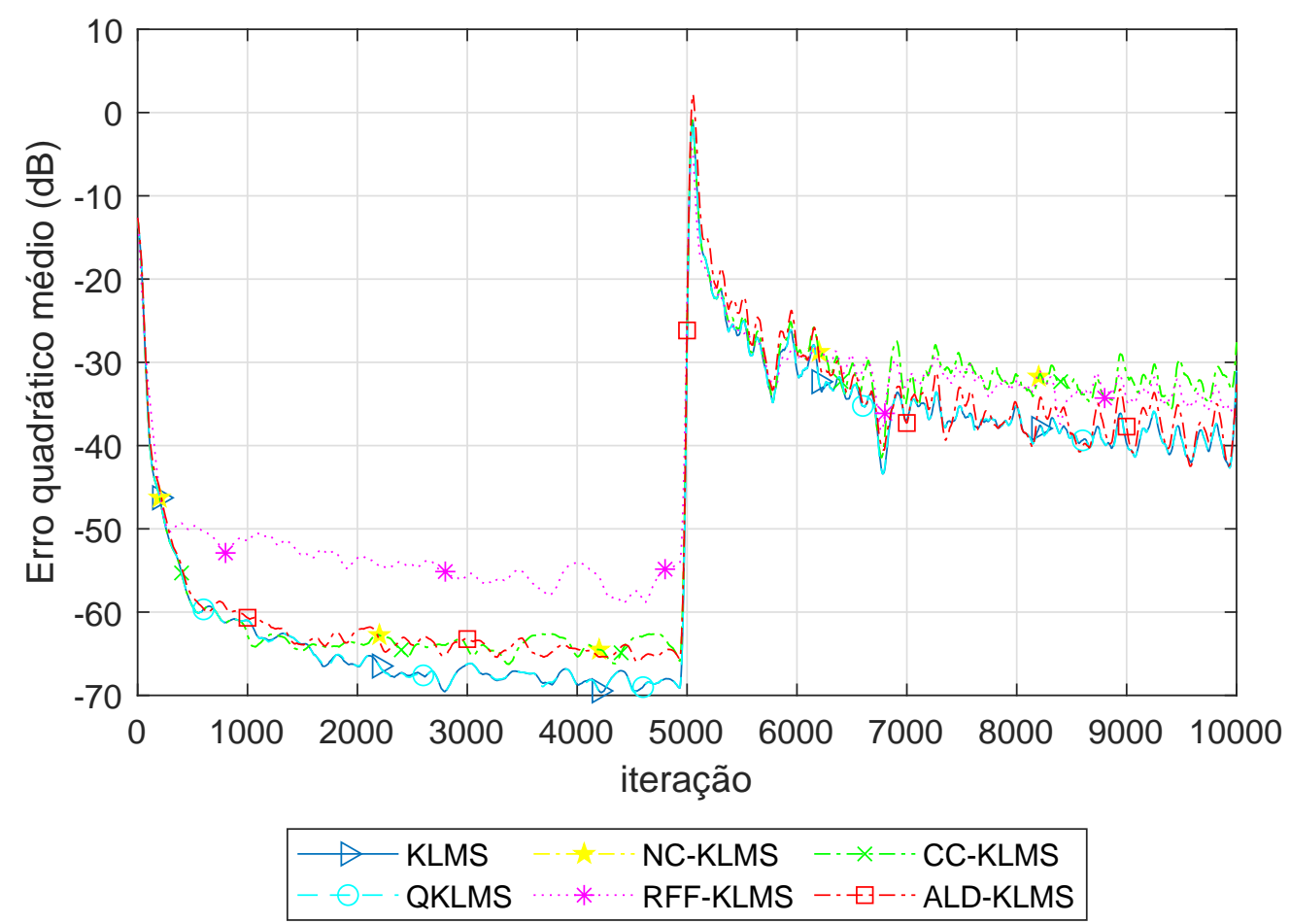

Figura 7: MSE para o problema de predição não linear considerando kernel gaussiano, $\zeta=1$, $M=7, \mu=0,5, \varepsilon_{N C}=\varepsilon_{Q}=0,01, \varepsilon_{C C}=0,9999, \varepsilon_{A L D}=0,0199$ e $D=5000$.

Fonte: Autor.

as técnicas de esparsificação realmente diminuem o custo computacional em relação ao KLMS. O número acumulado de multiplicações do KLMS na iteração $n=10^{4}$ é de aproximadamente $4,0 \times 10^{8}$ enquanto o do QKLMS é aproximadamente $1,2 \times 10^{8}$, o do ALD-KLMS é aproximadamente $3,3 \times 10^{8}$, o do RFF-KLMS $1,5 \times 10^{8}$ e o dos algoritmos NC-KLMS e CC-KLMS $1,2 \times 10^{8}$. Em compensação, o ALD-KLMS realiza $1,1 \times 10^{6}$ operações não lineares, comparado

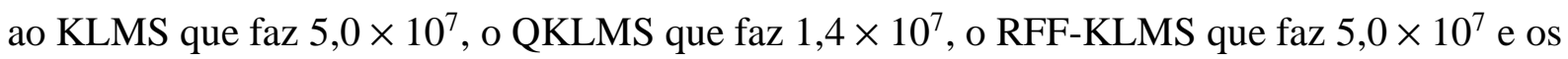
algoritmos NC-KLMS e CC-KLMS que realizam $2,9 \times 10^{7}$.

\subsection{Conclusão}

Neste capítulo foram apresentadas cinco técnicas de esparsificação de dicionário para métodos kernel, assim como os algoritmos de filtragem adaptativa do tipo KLMS baseados nessas técnicas. A simulação inicialmente realizada no Capítulo 1 foi revisitada e os algoritmos apresentados neste capítulo foram ajustados para ter aproximadamente o mesmo MSE do KLMS sem esparsificação. O NC-KLMS e o CC-KLMS tiveram resultados semelhantes, pois se utilizou o kernel gaussiano. Dessa forma, seus dicionários continham o mesmo número de elementos em todas as iterações. O QKLMS obteve um resultado melhor que os dois algoritmos 


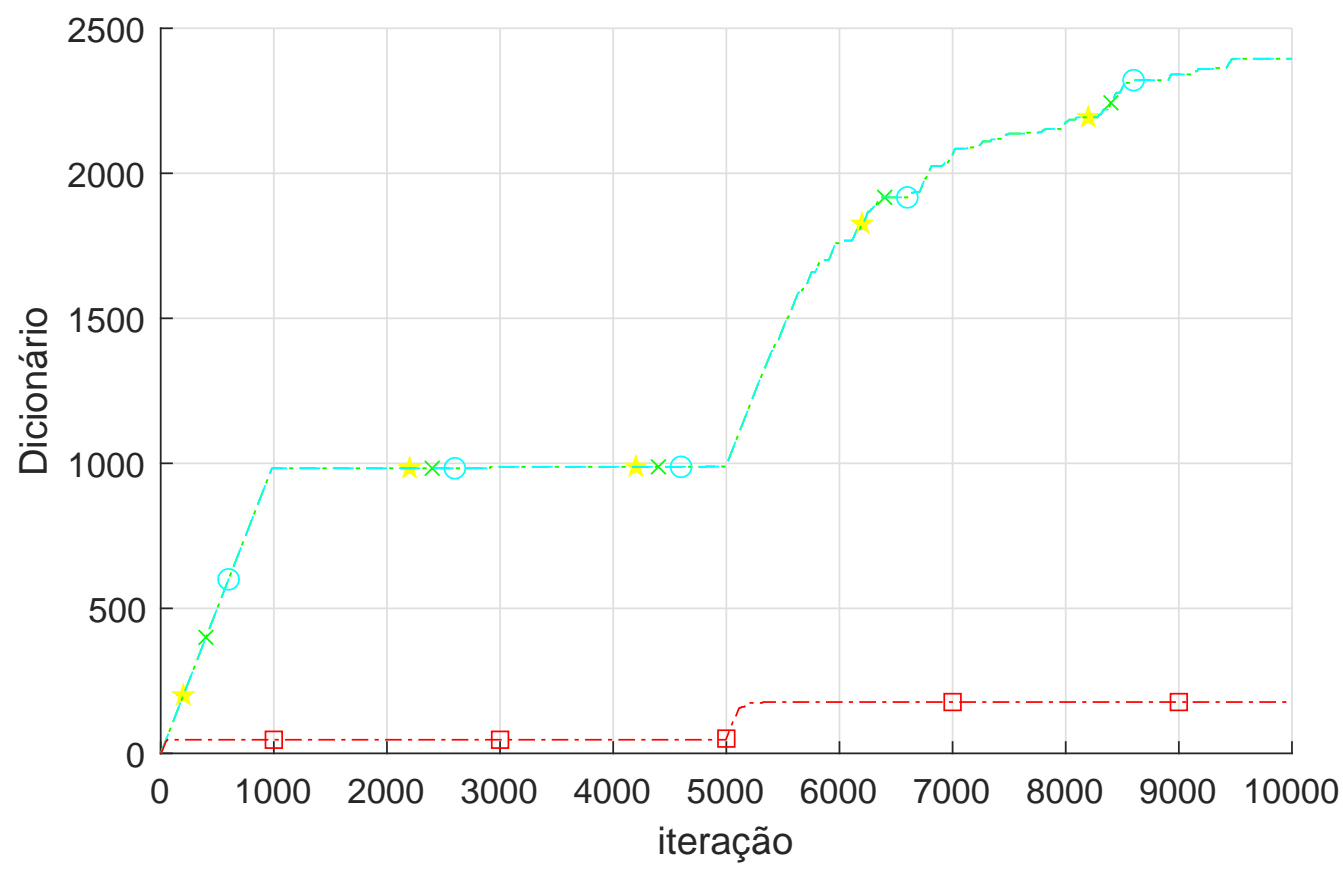

NC-KLMS $-\cdots * \cdots$ CC-KLMS $-\ominus-$ QKLMS $-\cdot \boxminus-\cdots$ ALD-KLMS

Figura 8: Tamanho dos dicionários para o problema de predição não linear considerando kernel gaussiano, $\zeta=1, M=7, \mu=0,5, \varepsilon_{N C}=\varepsilon_{Q}=0,01, \varepsilon_{C C}=0,9999, \varepsilon_{A L D}=0,0199$ e $D=5000$.

Fonte: Autor.

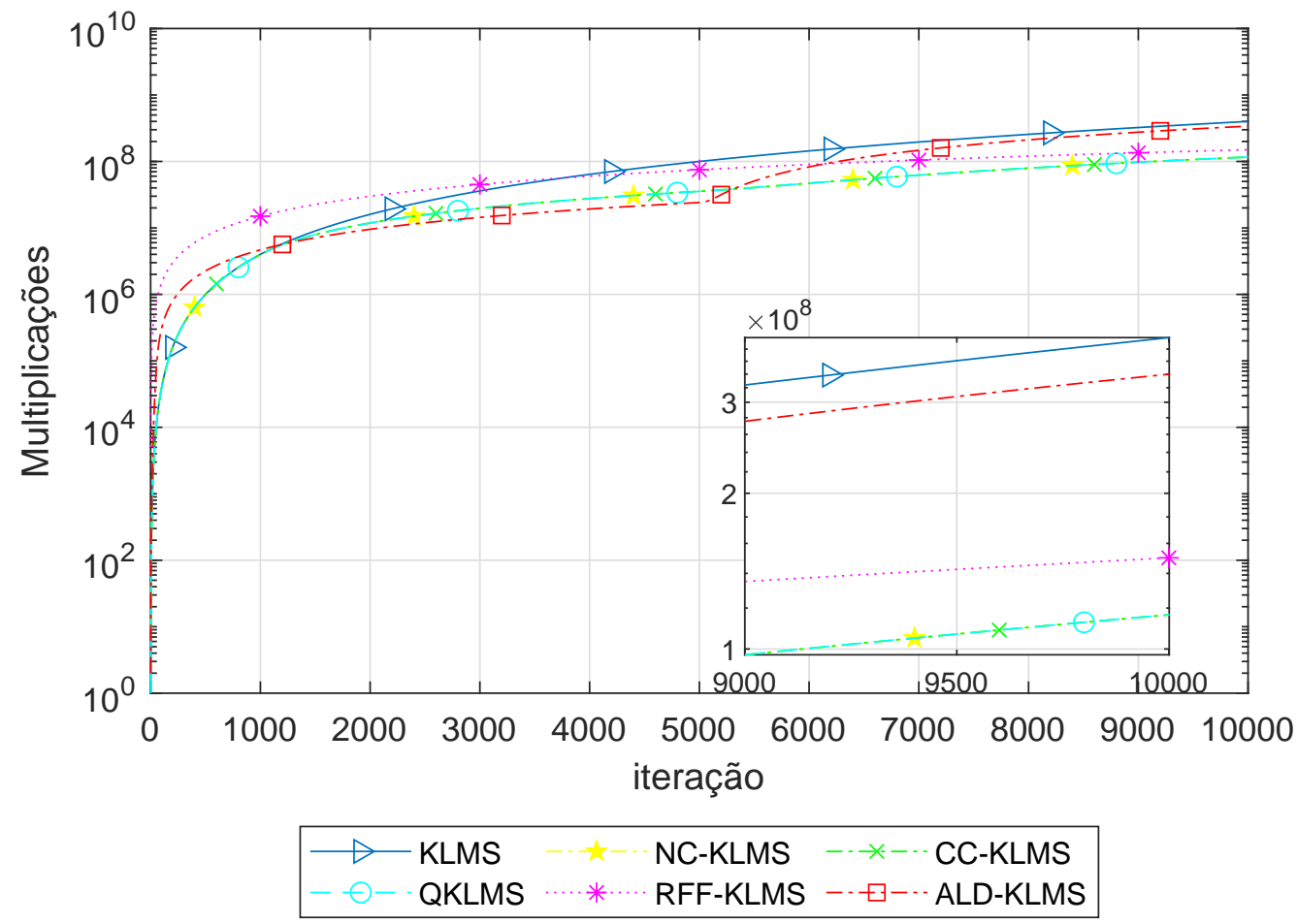

Figura 9: Número de operações de multiplicação acumuladas para o problema de predição não linear considerando kernel gaussiano, $\zeta=1, M=7, \mu=0,5, \varepsilon_{N C}=\varepsilon_{Q}=0,01, \varepsilon_{C C}=0,9999$, $\varepsilon_{A L D}=0,0199$ e $D=5000$. 


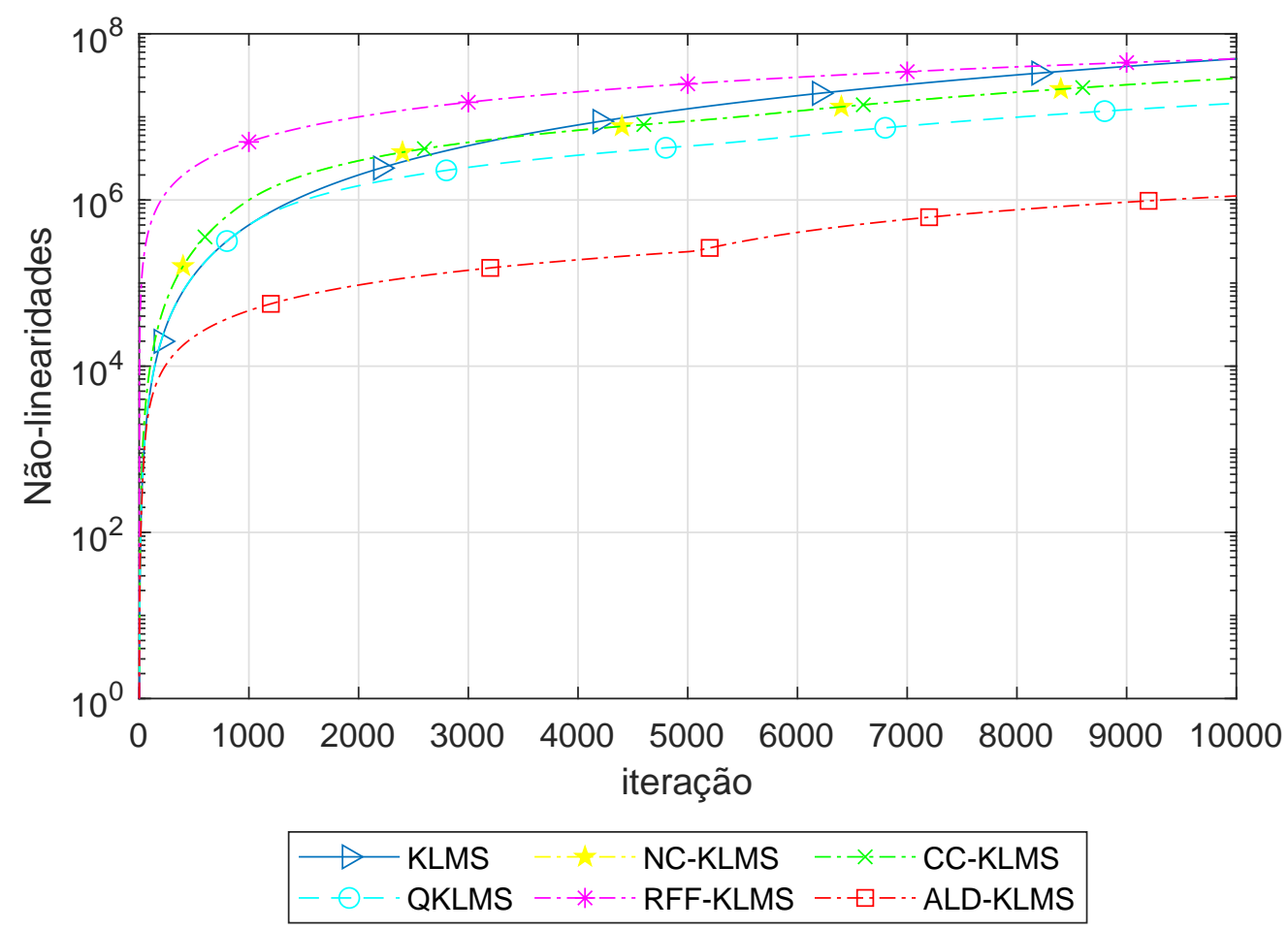

Figura 10: Número de operações não lineares acumuladas para o problema de predição não linear considerando kernel gaussiano, $\zeta=1, M=7, \mu=0,5, \varepsilon_{N C}=\varepsilon_{Q}=0,01, \varepsilon_{C C}=0,9999$, $\varepsilon_{A L D}=0,0199$ e $D=5000$.

Fonte: Autor.

já mencionados com o mesmo limiar de comparação do NC-KLMS. Isso se deve ao fato de o QKLMS alterar os parâmetros $a_{i}$, o que corrobora com o observado em (CHEN et al., 2012a). O RFF-KLMS apresentou um desempenho um pouco pior que os outros e não tem um dicionário para comparação. O ALD-KLMS conseguiu, mesmo com um dicionário bem menor que os das outras técnicas de esparsificação, um resultado comparável em termos de MSE. No entanto, é necessário ter cautela na escolha da técnica de esparsificação, pois um dicionário menor não significa um custo computacional menor. É o que foi observado no ALD-KLMS que apresenta um número maior de multiplicações acumuladas que os demais algoritmos, o que é compensado pelo número de não linearidades acumuladas. Dessa forma, apesar de ter um dicionário menor, o ALD apresenta um custo similar ao das demais técnicas de esparsificação consideradas neste capítulo.

Esses resultados de simulação mostram que é essencial acoplar uma técnica de esparsificação de dicionário ao KLMS. Em particular, verifica-se que o ALD-KLMS apresenta uma vantagem em termos de elementos de dicionário, mas seu custo computacional acumulado ainda é maior que o do QKLMS. Diante disso, inspirando-se no ALD, é proposto no próximo capítulo 
uma técnica de esparsificação que busca diminuir o custo acumulado, sem deteriorar muito o desempenho. 


\section{ORTOGONALIZAÇÃO DE GRAM-SCHMIDT PARA ESPARSIFICAÇÃO DE DICIONÁRIOS}

Neste capítulo, a técnica de esparsificação de dicionários para métodos baseados em kernel é proposta. Primeiramente, os conjuntos envolvidos são abordados. Em seguida, uma forma para se obter uma base ortonormal para um subespaço do espaço induzido pelo kernel é apresentada. A utilização dessa base como uma técnica de esparsificação é introduzida e um limite para o erro no produto interno quando ela é utilizada é derivado. Por último, apresenta-se uma simulação para mostrar o funcionamento da técnica.

\subsection{Conjuntos envolvidos}

Como apresentado na introdução, um método baseado em kernel mapeia vetores de um espaço de entrada $\mathcal{U} \in \mathbb{R}^{M}$ em um espaço de saída $\mathcal{H}$ por meio de uma transformação $\varphi(\cdot)$ tal que $\langle\varphi(\mathbf{u}), \varphi(\mathbf{v})\rangle_{\mathcal{H}}=\varphi(\mathbf{u})^{T} \varphi(\mathbf{v})=\kappa(\mathbf{u}, \mathbf{v})$.

Em geral, o espaço de entrada $\mathcal{U}$ não coincide com o $\mathbb{R}^{M}$ e portanto, há um conjunto de saída $\mathcal{S}$ para o qual os vetores de $\mathcal{U}$ são mapeados. Na Figura 11, é mostrado o diagrama de Venn dos conjuntos envolvidos. Nessa figura, os espaços vetoriais são representados por linhas grossas, enquanto os conjuntos imagem por linhas finas.

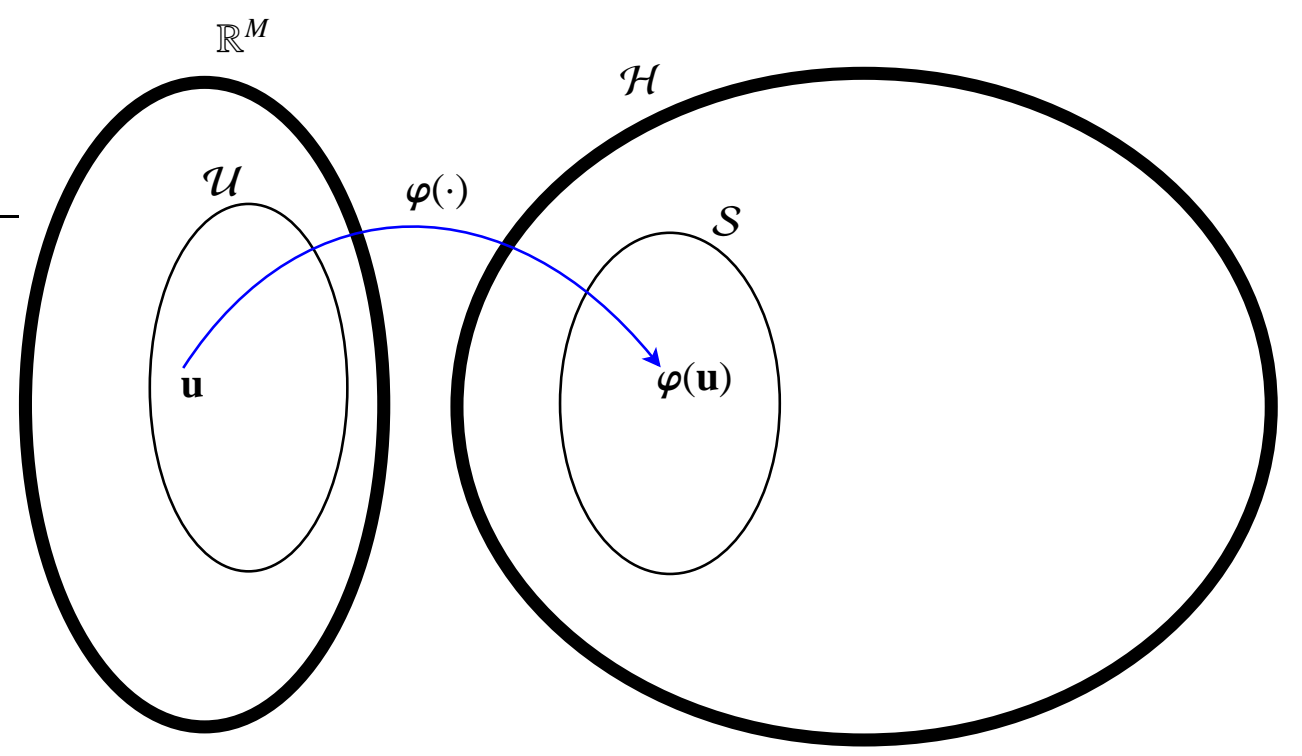

Figura 11: Diagrama de Venn dos espaços vetoriais e conjuntos imagem envolvidos.

Fonte: Autor. 
Em geral, os métodos baseados em kernel utilizam um dicionário $\mathcal{D}$ formado a partir de vetores de entrada $\mathbf{u} \in \mathcal{U}$ tendo $\varphi(\mathcal{D}) \in \mathcal{S}$ como imagem. Na Figura 12, é mostrado o diagrama de Venn considerando esses conjuntos, sendo que os conjuntos representados por linhas tracejadas possuem um número finito de elementos.

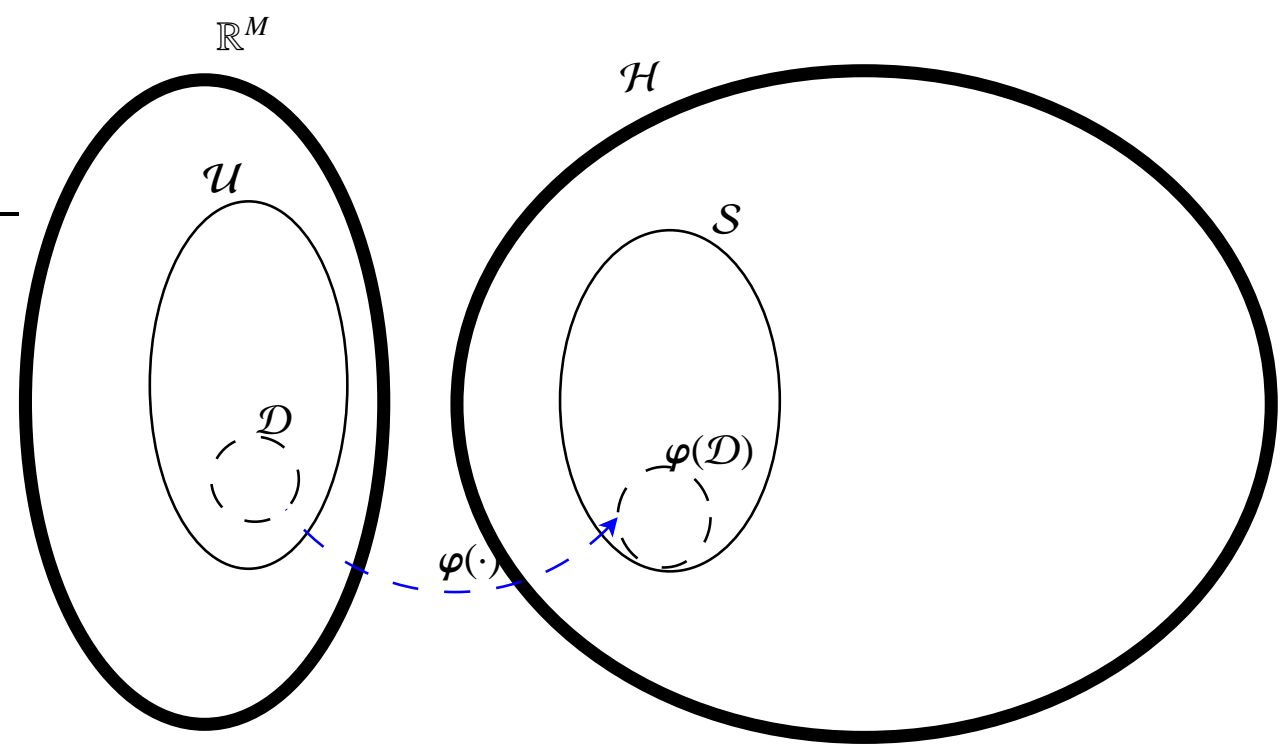

Figura 12: Diagrama de Venn dos espaços vetoriais e conjuntos imagem envolvidos considerando o dicionário $\mathcal{D}$ e sua imagem $\varphi(\mathcal{D})$.

Fonte: Autor.

Apesar de nem sempre ser possível encontrar uma base para $\mathcal{S}$, é possível utilizar vetores do conjunto $\varphi(\mathcal{D})$ para criar uma base ortonormal $\mathbf{B}$ de um espaço $\mathcal{B} \subset \mathcal{H}$, que contém as projeções dos vetores do conjunto $\mathcal{S}$, como esquematizado na Figura 13. A base $\mathcal{B}$ pode ser obtida pelo processo de ortogonalização de Gram-Schmidt (MEYER, 2000), o que será abordado na seção seguinte. 


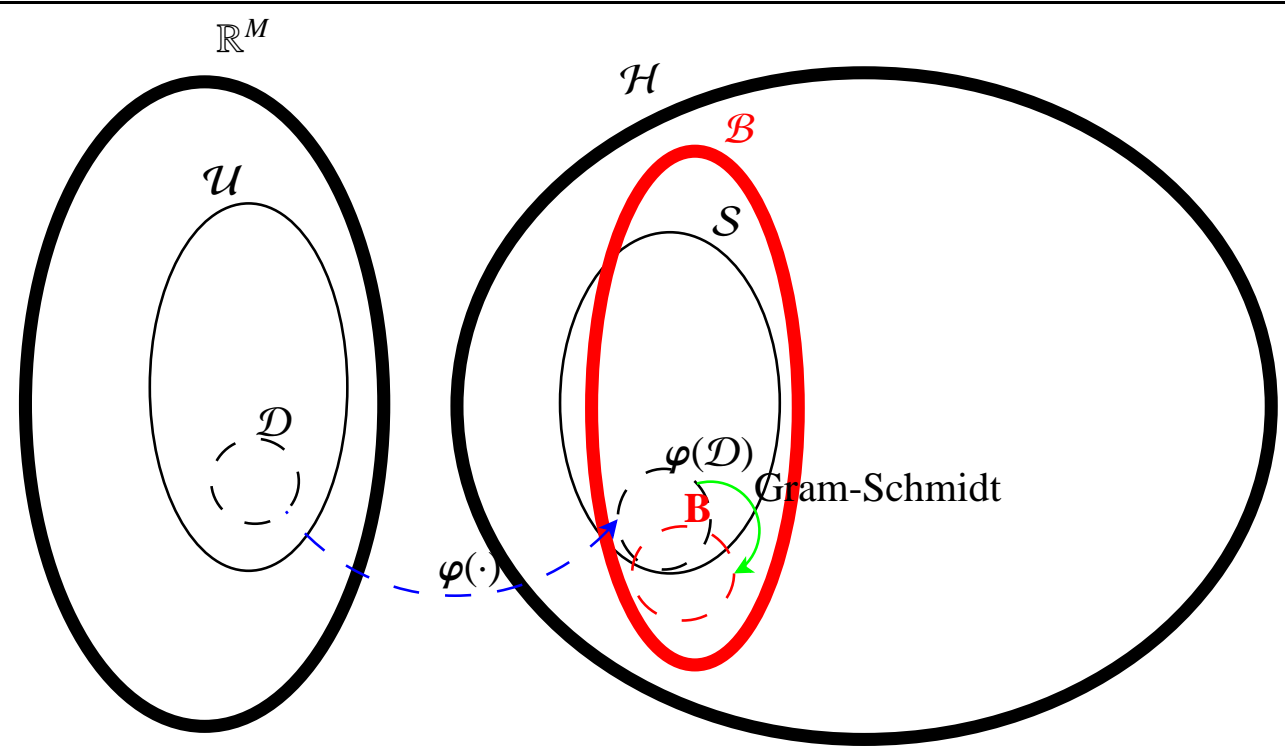

Figura 13: Diagrama de Venn dos espaços vetoriais e conjuntos imagem envolvidos considerando o dicionário e o espaço vetorial $\mathcal{B}$.

Fonte: Autor.

\subsection{Representando os vetores mapeados como projeções de dimensão finita}

Seja $\mathcal{D} \triangleq\left\{\mathbf{u}_{1}, \mathbf{u} 2, \cdots, \mathbf{u}_{N}\right\}$ um dicionário cujos vetores mapeados $\varphi(\mathcal{D}) \triangleq$ $\left\{\varphi\left(\mathbf{u}_{1}\right), \varphi\left(\mathbf{u}_{2}\right), \cdots, \varphi\left(\mathbf{u}_{N}\right)\right\}$ formam um conjunto linearmente independente. Usando $\varphi(\mathcal{D})$ é possível gerar um conjunto ortonormal $\mathbf{B}_{N} \triangleq\left\{\boldsymbol{\beta}_{1}, \boldsymbol{\beta}_{2}, \cdots, \boldsymbol{\beta}_{N}\right\}$ que é a base de um espaço vetorial $\mathcal{B} \subset \mathcal{H}$. A projeção ortogonal de $\varphi\left(\mathbf{u}_{\ell}\right)$ em $\mathcal{B}$, denotada como o vetor $\mathbf{p}_{\ell} \triangleq \operatorname{proj}_{\mathcal{B}} \varphi\left(\mathbf{u}_{\ell}\right) \in \mathcal{B}$, é composta de componentes na direção de cada vetor $\left\{\boldsymbol{\beta}_{i}\right\}$ da base como

$$
\mathbf{p}_{\ell}=\sum_{i=1}^{N}\left\langle\varphi\left(\mathbf{u}_{\ell}\right), \boldsymbol{\beta}_{i}\right\rangle_{\mathcal{H}} \boldsymbol{\beta}_{i}=\sum_{i=1}^{N} p_{\ell, i} \boldsymbol{\beta}_{i},
$$

em que se definiu $p_{\ell, i} \triangleq\left\langle\varphi\left(\mathbf{u}_{\ell}\right), \boldsymbol{\beta}_{i}\right\rangle_{\mathcal{H}}$. Se $\mathbf{u}_{\ell}$ coincidir com um dos vetores de $\mathcal{D}, \varphi\left(\mathbf{u}_{\ell}\right)=\mathbf{p}_{\ell}$ já que $\varphi\left(\mathbf{u}_{\ell}\right)$ é usado para criar a base $\mathbf{B}_{N}$. Caso contrário, $\varphi\left(\mathbf{u}_{\ell}\right)=\mathbf{p}_{\ell}+\mathbf{p}_{\ell}^{\perp}$, em que $\mathbf{p}_{\ell}^{\perp} \neq \mathbf{0}$ é o componente de $\varphi\left(\mathbf{u}_{\ell}\right)$ ortogonal a $\mathcal{B}$.

Os componentes $p_{\ell, i}, i=1,2, \cdots, N$, de (3.1) podem ser agrupados no vetor

$$
\widetilde{\mathbf{p}}_{\ell} \triangleq\left[\begin{array}{llll}
p_{\ell, 1} & p_{\ell, 2} & \cdots & p_{\ell, N}
\end{array}\right]^{T},
$$

que pode ser usado como uma representação em dimensão finita do vetor mapeado $\varphi\left(\mathbf{u}_{\ell}\right)$, que inclusive pode ter dimensão infinita. Na Figura 14, é mostrado um exemplo dessa representação considerando $N=2$. 


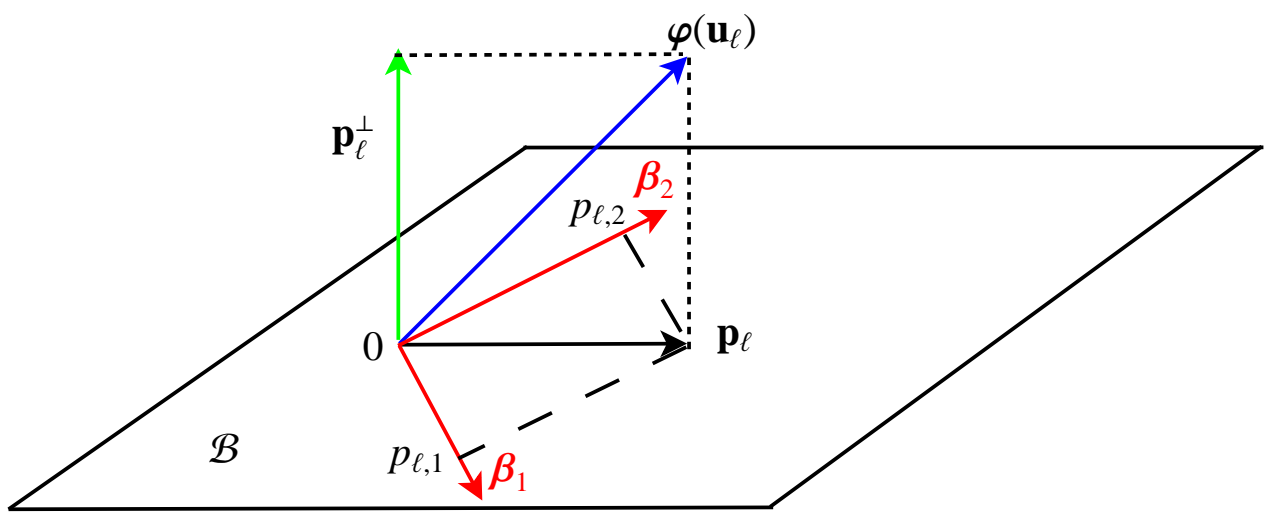

Figura 14: Representação do vetor $\varphi\left(u_{\ell}\right)$ a partir dos componentes de sua projeção para $N=2$. Fonte: Autor.

A vantagem em usar uma base ortonormal é que com ela é possível calcular o produto interno de uma forma concisa (MEYER, 2000). Dados dois vetores $\mathbf{p}_{\ell}, \mathbf{p}_{k} \in \mathcal{B}$, o produto interno entre eles pode ser calculado como

$$
\left\langle\mathbf{p}_{\ell}, \mathbf{p}_{k}\right\rangle=\sum_{i=1}^{N} \sum_{j=1}^{N} p_{\ell, i} p_{k, j}\left\langle\boldsymbol{\beta}_{i}, \boldsymbol{\beta}_{j}\right\rangle=\widetilde{\mathbf{p}}_{\ell}^{T} \widetilde{\mathbf{p}}_{k},
$$

em que a última igualdade é consequência da ortonormalidade da base. Com isso, a técnica apresentada neste capítulo realiza menos operações de multiplicação que o ALD-KLMS onde para realizar a operação de multiplicação entre vetores é necessário utilizar o gramiano, como visto na Equação (2.25) da página 16.

Em filtragem adaptativa baseada em kernel, essa representação pode substituir $\varphi(\mathbf{u}(n))$ no KLMS (Equações (1.4) e (1.6) da página 3). Nesse caso, a dimensão do vetor de pesos do filtro fica igual à cardinalidade do dicionário $N(n)$, independente do kernel associado. Na sequência, calcula-se $\widetilde{\mathbf{p}}_{\ell}$ sem se conhecer explicitamente $\varphi(\cdot)$.

\subsection{Forma matricial do processo de Gram-Schmidt}

Para se obter uma base ortonormal, pode-se usar o processo de ortogonalização de GramSchmidt, que calcula os vetores da base a partir dos vetores de um conjunto gerador não orto- 
gonal. Esse processo é descrito pelas equações (MEYER, 2000)

$$
\begin{aligned}
& \boldsymbol{\beta}_{1}=\frac{\varphi\left(\mathbf{u}_{\mathbf{1}}\right)}{\left\|\boldsymbol{\varphi}\left(\mathbf{u}_{\mathbf{1}}\right)\right\|}, \\
& \boldsymbol{\beta}_{m} \triangleq \frac{\gamma_{m}}{\left\|\boldsymbol{\gamma}_{m}\right\|}, \\
& \boldsymbol{\gamma}_{m} \triangleq \varphi\left(\mathbf{u}_{m}\right)-\sum_{i=1}^{m-1}\left\langle\varphi\left(\mathbf{u}_{m}\right), \boldsymbol{\beta}_{i}\right\rangle_{\mathcal{H}} \boldsymbol{\beta}_{i},
\end{aligned}
$$

$m=2,3, \cdots, N$. Os vetores da base podem ser escritos como uma combinação linear dos vetores do dicionário mapeados (MEYER, 2000), ou seja,

$$
\boldsymbol{\beta}_{i}=\sum_{j=1}^{N} h_{i j} \varphi\left(\mathbf{u}_{j}\right), \quad i=1,2, \cdots, N .
$$

De (3.5) e (3.6), observa-se que o vetor $\boldsymbol{\beta}_{m}$, só depende dos vetores $\varphi\left(\mathbf{u}_{j}\right), j=1,2, \cdots, m$. Assim, $h_{i j}=0$ para $j>i$ em (3.7).

Substituindo (3.7) em (3.1) e usando as propriedades lineares do produto interno, chega-se a

$$
\mathbf{p}_{\ell}=\sum_{i=1}^{N} \sum_{j=1}^{N} h_{i j}\left\langle\boldsymbol{\varphi}\left(\mathbf{u}_{\ell}\right), \boldsymbol{\varphi}\left(\mathbf{u}_{j}\right)\right\rangle_{\mathcal{H}} \boldsymbol{\beta}_{i} .
$$

Usando o truque do kernel, dado por (1.1), obtém-se

$$
\mathbf{p}_{\ell}=\sum_{i=1}^{N} \sum_{j=1}^{N} h_{i j} \kappa\left(\mathbf{u}_{\ell}, \mathbf{u}_{j}\right) \boldsymbol{\beta}_{i} .
$$

Comparando (3.9) com (3.1), conclui-se que

$$
p_{\ell, i}=\sum_{j=1}^{N} h_{i j} \kappa\left(\mathbf{u}_{\ell}, \mathbf{u}_{j}\right) .
$$

Usando a definição do vetor $\boldsymbol{\kappa}_{N}\left(\mathbf{u}_{\ell}\right)$ em (2.17) na página 15 e as matrizes triangulares inferiores

$$
\mathbf{H}_{i}=\left[\begin{array}{c}
\mathbf{h}_{1 i} \\
\mathbf{h}_{2 i} \\
\vdots \\
\mathbf{h}_{i i}
\end{array}\right] \triangleq\left[\begin{array}{cccc}
h_{11} & 0 & \ldots & 0 \\
h_{21} & h_{22} & \ldots & 0 \\
\vdots & \vdots & \ddots & \vdots \\
h_{i 1} & h_{i 2} & \ldots & h_{i i}
\end{array}\right]
$$

$i=1,2, \cdots, N$, em conjunto com (3.10), o vetor $\widetilde{\mathbf{p}}_{\ell}$ de (3.2) pode ser escrito como

$$
\widetilde{\mathbf{p}}_{\ell}=\mathbf{H}_{N} \boldsymbol{\kappa}_{N}\left(\mathbf{u}_{\ell}\right)
$$

Para realizar esse cálculo, ainda é preciso calcular a matriz $\mathbf{H}_{N}$. 
Substituindo (3.7) em (3.6) e usando o truque do kernel de (1.1), obtem-se

$$
\boldsymbol{\gamma}_{m}=\boldsymbol{\varphi}\left(\mathbf{u}_{m}\right)-\sum_{i=1}^{m-1} \sum_{j=1}^{N} h_{i j} \kappa\left(\mathbf{u}_{m}, \mathbf{u}_{j}\right) \sum_{r=1}^{N} h_{i r} \boldsymbol{\varphi}\left(\mathbf{u}_{r}\right) .
$$

Definindo

$$
\bar{h}_{m r} \triangleq\left\{\begin{array}{ll}
-\sum_{i=1}^{m-1} \sum_{j=1}^{N} h_{i j} h_{i r} \kappa\left(\mathbf{u}_{m}, \mathbf{u}_{j}\right), & 1 \leq r<m \\
1, & r=m \\
0, & r>m
\end{array},\right.
$$

a Equação (3.13) pode ser escrita como

$$
\boldsymbol{\gamma}_{m}=\sum_{r=1}^{N} \bar{h}_{m r} \boldsymbol{\varphi}\left(\mathbf{u}_{r}\right)
$$

Substituindo (3.15) no numerador de (3.5), tem-se

$$
\boldsymbol{\beta}_{m}=\frac{1}{\left\|\boldsymbol{\gamma}_{m}\right\|} \sum_{r=1}^{N} \bar{h}_{m r} \boldsymbol{\varphi}\left(\mathbf{u}_{r}\right)
$$

Comparando (3.16) com (3.7), identifica-se que $h_{m r}=\bar{h}_{m r} /\left\|\gamma_{m}\right\|$. Assim, os vetores linha

$$
\overline{\mathbf{h}}_{m i} \triangleq\left[\begin{array}{lllll}
\bar{h}_{m 1} & \bar{h}_{m 2} & \cdots & \bar{h}_{m(r-1)} & \bar{h}_{m i}
\end{array}\right]
$$

estão relacionados com as linhas da matriz $\mathbf{H}_{i}$ por

$$
\mathbf{h}_{m i}=\overline{\mathbf{h}}_{m i} /\left\|\gamma_{m}\right\|
$$

De (3.4) e (3.7), nota-se que $\mathbf{H}_{1}=h_{11}=1 /\left\|\varphi\left(\mathbf{u}_{1}\right)\right\|$. Além disso, de (3.1), observa-se que a matriz $\mathbf{H}_{i+1}$ de dimensão $(i+1) \times(i+1)$ pode ser obtida a partir da matriz $\mathbf{H}_{i}$, acrescentando-se um vetor coluna de zeros de dimensão $i$, denotado por $\mathbf{0}_{i \times 1}$, e o vetor $\mathbf{h}_{(i+1)(i+1)}$ da forma

$$
\mathbf{H}_{i+1}=\left[\begin{array}{cc}
\mathbf{H}_{i} & \mathbf{0}_{i \times 1} \\
\mathbf{h}_{(i+1)(i+1)}
\end{array}\right] .
$$

Com isso, é possível calcular as matrizes $\mathbf{H}_{i}$ de forma iterativa. A partir de (3.14), (2.17) e (3.11) o vetor $\overline{\mathbf{h}}_{m i}$ pode ser calculado como

$$
\overline{\mathbf{h}}_{(i+1)(i+1)}=\left[\begin{array}{ll}
-\boldsymbol{\kappa}_{i}^{T}\left(\mathbf{u}_{i+1}\right) \mathbf{H}_{i}^{T} \mathbf{H}_{i} & 1
\end{array}\right] .
$$

De (3.18), verifica-se que para calcular o vetor $\mathbf{h}_{(i+1)(i+1)}$ ainda é preciso calcular $\left\|\gamma_{i+1}\right\|$. 
Usando (3.15), as propriedades lineares do produto interno e o truque do kernel de (1.1), chegase a

$$
\left\|\boldsymbol{\gamma}_{i+1}\right\|=\sqrt{\sum_{k=1}^{i+1} \sum_{\ell=1}^{i+1} \bar{h}_{(i+1) k} \bar{h}_{(i+1) \ell} \kappa\left(\mathbf{u}_{k}, \mathbf{u}_{\ell}\right) .}
$$

Essa norma pode ser reescrita em termos do gramiano definido na Equação (2.18) da página 16. Para isso, cabe notar que o gramiano também pode ser calculado de maneira recursiva como mostrado na Equação (2.27) da página 13. Dessa equação, chega-se à

$$
\mathbf{G}_{i+1}=\left[\begin{array}{cc}
\mathbf{G}_{i} & \boldsymbol{\kappa}_{i}\left(\mathbf{u}_{i+1}\right) \\
\boldsymbol{\kappa}_{i}^{T}\left(\mathbf{u}_{i+1}\right) & \kappa\left(\mathbf{u}_{i+1}, \mathbf{u}_{i+1}\right)
\end{array}\right],
$$

em que $G_{1}=k\left(u_{1}, u_{1}\right)$. Assim, usando (3.22) e (3.17), reescreve-se (3.21) como

$$
\left\|\gamma_{i+1}\right\|=\sqrt{\overline{\mathbf{h}}_{(i+1)(i+1)} \cdot \mathbf{G}_{i+1} \cdot \overline{\mathbf{h}}_{(i+1)(i+1)}^{T}} .
$$

Usando (3.18), (3.19), (3.20), (3.22) e (3.23), obtém-se $\mathbf{H}_{i+1}$. Repetindo-se esse procedimento de forma iterativa, chega-se a $\mathbf{H}_{N}$ e a partir de (3.12) calcula-se $\widetilde{\mathbf{p}_{\ell}}$.

É importante observar que não é necessário conhecer explicitamente os vetores da base $\mathbf{B}_{N}$ nem os vetores mapeados $\varphi\left(\mathbf{u}_{i}\right), i=1,2, \cdots, N$ para se calcular $\widetilde{\mathbf{p}_{\ell}}$.

\subsection{Relações entre as matrizes $\mathbf{H}_{i}$ e $\mathbf{G}_{i}$}

Algumas relações entre as matrizes $\mathbf{H}_{i}$ e $\mathbf{G}_{i}$ são necessárias para se chegar à técnica esparsificação de dicionários proposta neste capítulo e também são usadas para retirar elementos do dicionário, que será abordado no Capítulo 4. Essas relações são tratadas a seguir.

Partindo da definição do gramiano (2.18) da página 16, e multiplicando essa matriz pela esquerda por $\mathbf{H}_{i}$, tem-se

$$
\mathbf{H}_{i} \mathbf{G}_{i}=\left[\begin{array}{llll}
\widetilde{\mathbf{p}}_{1} & \widetilde{\mathbf{p}}_{2} & \cdots & \widetilde{\mathbf{p}}_{i}
\end{array}\right] \triangleq \widetilde{\mathbf{P}}_{i}
$$

Cada coluna da matriz $\widetilde{\mathbf{P}}_{i}$ contém a representação dos vetores mapeados, $\varphi(\mathcal{D})$. Como esses vetores foram utilizados na obtenção da base ortonormal, sua representação por $\widetilde{\mathbf{p}}_{k}, k=1,2, \cdots i$ é perfeita, ou seja, a projeção no espaço $\mathcal{B}$ é o próprio vetor. Dessa forma, se essas representações forem utilizadas para calcular os produtos internos entre os vetores, se obtém a matriz gramiano novamente, isto é

$$
\widetilde{\mathbf{P}}_{i}^{T} \widetilde{\mathbf{P}}_{i}=\mathbf{G}_{i} .
$$


A matriz $\widetilde{\mathbf{P}}_{i}$ é triangular superior, já que a representação de cada vetor utilizado no processo de Gram-Shimidt só depende dele mesmo e dos vetores que foram utilizados antes dele. Assim, $\widetilde{\mathbf{P}}_{i}^{T}$ é uma matriz triangular inferior e portanto a matriz $\mathbf{G}_{i}$ tem uma decomposição na forma $\mathbf{L} \mathbf{L}^{T}$, em que L é uma matriz triangular inferior. Essa decomposição é conhecida como decomposição de Cholesky e é única quando a matriz decomposta é simétrica e positiva semi-definida, como é o caso (MEYER, 2000).

Ao substituir a definição de $\widetilde{\mathbf{P}}_{i}$, dada em (3.24), na Equação em (3.25), tem-se

$$
\mathbf{G}_{i}^{T} \mathbf{H}_{i}^{T} \mathbf{H}_{i} \mathbf{G}_{i}=\mathbf{G}_{i}
$$

Multiplicando (3.26) à esquerda e à direita pela inversa do gramiano, obtém-se

$$
\mathbf{H}_{i}^{T} \mathbf{H}_{i}=\mathbf{G}_{i}^{-1}
$$

Multiplicando (3.27) à direita por $\mathbf{G}_{i}$ e usando a definição de $\widetilde{\mathbf{P}}_{i}$, tem-se

$$
\mathbf{H}_{i}^{T} \widetilde{\mathbf{P}}_{i}=\mathbf{I},
$$

sendo I é a matriz identidade.

\subsection{Selecionando vetores para a base}

O procedimento para calcular a representação de dimensão finita dos vetores mapeados pode ser usado como uma técnica de esparsificação. A ideia é decidir se o vetor de entrada $\mathbf{u}_{\ell} \notin \mathcal{D}$ deve ou não ser incluído no dicionário. Para isso, verifica-se se os vetores do conjunto aumentado $\varphi(\mathcal{D}) \cup \varphi\left(\mathbf{u}_{\ell}\right)$ são próximos de serem linearmente dependentes, o que pode ser medido pelo determinante do gramiano (MEYER, 2000).

Seja $\mathbf{G}_{N}$ o gramiano do conjunto $\varphi(\mathcal{D})$. Usando (3.22), o gramiano de $\varphi(\mathcal{D}) \cup \varphi\left(\mathbf{u}_{\ell}\right)$ pode ser expresso como

$$
\widetilde{\mathbf{G}}=\left[\begin{array}{cc}
\mathbf{G}_{N} & \boldsymbol{\kappa}_{N}\left(\mathbf{u}_{\ell}\right) \\
\boldsymbol{\kappa}_{N}^{T}\left(\mathbf{u}_{\ell}\right) & \kappa\left(\mathbf{u}_{\ell}, \mathbf{u}_{\ell}\right)
\end{array}\right]
$$

e seu determinante pode ser calculado como (MEYER, 2000)

$$
|\widetilde{\mathbf{G}}|=\left|\mathbf{G}_{N}\right|\left[\kappa\left(\mathbf{u}_{\ell}, \mathbf{u}_{\ell}\right)-\boldsymbol{\kappa}_{N}^{T}\left(\mathbf{u}_{\ell}\right) \mathbf{G}_{N}^{-1} \boldsymbol{\kappa}_{N}\left(\mathbf{u}_{\ell}\right)\right]
$$

Lembrando que $\kappa\left(\mathbf{u}_{\ell}, \mathbf{u}_{\ell}\right)=\left\|\varphi\left(\mathbf{u}_{\ell}\right)\right\|^{2}$ e usando (3.27) e (3.12), pode-se reescrever (3.30) 
como

$$
|\widetilde{\mathbf{G}}|=\left|\mathbf{G}_{N}\right|\left[\left\|\varphi\left(\mathbf{u}_{\ell}\right)\right\|^{2}-\left\|\mathbf{p}_{\ell}\right\|^{2}\right]
$$

Quando $|\widetilde{\mathbf{G}}|$ é nulo, o conjunto aumentado $\varphi(\mathcal{D}) \cup \varphi\left(\mathbf{u}_{\ell}\right)$ é linearmente dependente e $\mathbf{u}_{\ell}$ pode ser representado na base $\mathbf{B}_{N}$. Esse fato pode ser usado para decidir se $\mathbf{u}_{\ell}$ deve ser incluído ou não no dicionário. Como os vetores de $\varphi(\mathcal{D})$ são linearmente independentes, $\left|\mathbf{G}_{N}\right|>0$. Portanto, usando um limiar $\varepsilon_{G S}>0$, se

$$
\left\|\varphi\left(\mathbf{u}_{\ell}\right)\right\|^{2}-\left\|\mathbf{p}_{\ell}\right\|^{2}=\left\|\mathbf{p}_{\ell}^{\perp}\right\|^{2}=\kappa\left(\mathbf{u}_{\ell}, \mathbf{u}_{\ell}\right)-\widetilde{\mathbf{p}}_{\ell}^{T} \widetilde{\mathbf{p}}_{\ell}<\varepsilon_{G S}
$$

for satisfeito, conjectura-se que o conjunto aumentado $\varphi(\mathcal{D}) \cup \varphi\left(\mathbf{u}_{\ell}\right)$ é próximo de ser linearmente dependente e $\mathbf{u}_{\ell}$ não é incluído no dicionário. Caso contrário, inclui-se $\mathbf{u}_{\ell}$ no dicionário e calculam-se as matrizes $\mathbf{G}_{N+1}$ e $\mathbf{H}_{N+1}$. É possível notar a semelhança entre esse critério e o critério do ALD-KLMS da Equação (2.22) da página 16. Apesar de terem sido propostos por caminhos diferentes, chega-se ao mesmo critério de esparsificação. No entanto, a interpretação geométrica deixa explícita aqui a necessidade de que a componente ortogonal deve ter norma quadrática menor que o limiar para incluir o vetor $\mathbf{u}_{\ell}$ no dicionário.

Para o kernel gaussiano, $\kappa\left(\mathbf{u}_{\ell}, \mathbf{u}_{\ell}\right)=1$ e (3.32) é reduzida a

$$
\widetilde{\mathbf{p}}_{\ell}^{T} \widetilde{\mathbf{p}}_{\ell}>1-\varepsilon_{G S}
$$

Como $0<\widetilde{\mathbf{p}}_{\ell}^{T} \widetilde{\mathbf{p}}_{\ell} \leq 1$, o limiar deve ser escolhido no intervalo $0 \leq \varepsilon_{G S} \leq 1$, o que simplifica o ajuste desse parâmetro. Para $\varepsilon_{G S}=1$, apenas o vetor $u_{1}$ é incluído no dicionário enquanto que para $\varepsilon_{G S}=0$ o dicionário contém todos os vetores de entrada.

A Figura 15(a) mostra um vetor cuja representação não é apropriada, ou seja, $\left\|\mathbf{p}^{\perp}\right\|^{2} \geq \varepsilon_{G S}$, enquanto a Figura 15(b) mostra um vetor com representação apropriada, ou seja, com $\left\|\mathbf{p}^{\perp}\right\|^{2}<$ $\varepsilon_{G S}$. Os vetores com projeção não apropriada devem ser incluídos no dicionário. 


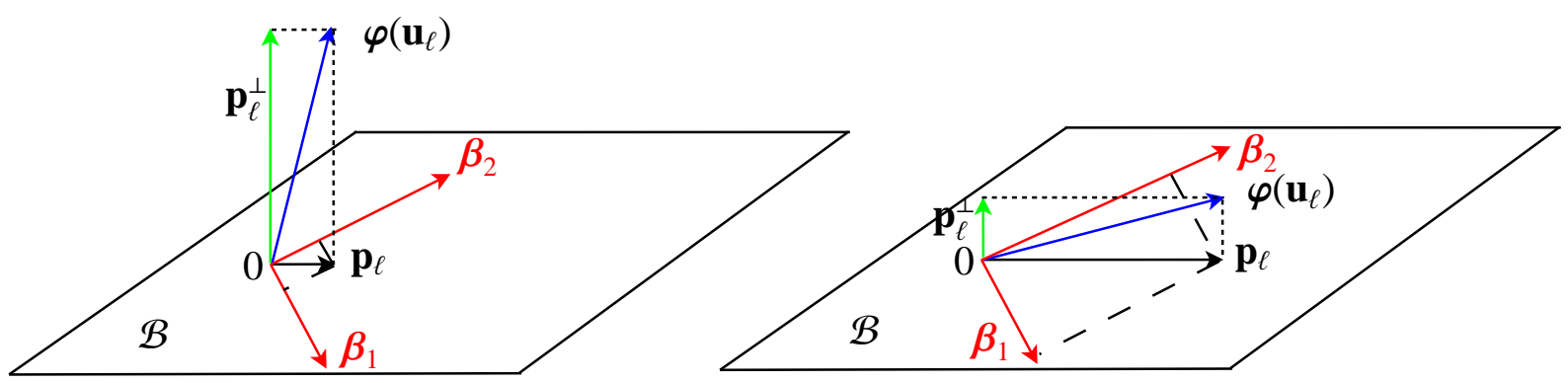

(a)

(b)

Figura 15: Representação não apropriada (a) e apropriada (b) dos vetores a partir dos componentes de sua projeção.

Fonte: Autor.

\subsection{Erro do produto interno}

O uso da técnica explicada anteriormente induz que os produtos internos $\varphi^{T}\left(\mathbf{u}_{i}\right) \varphi\left(\mathbf{u}_{j}\right)$ sejam aproximados por $\widetilde{\mathbf{p}}_{i}^{T} \widetilde{\mathbf{p}}_{j}, 0<i, j \leq n$. Quando $\mathbf{u}_{i}, \mathbf{u}_{j} \in \mathcal{D}$ não há aproximações já que nesse caso os vetores mapeados foram usados para criar a base. Em contrapartida, quando $\mathbf{u}_{i}, \mathbf{u}_{j} \notin \mathcal{D}$, observa-se que

$$
\boldsymbol{\varphi}\left(\mathbf{u}_{i}\right)^{T} \boldsymbol{\varphi}\left(\mathbf{u}_{j}\right)=\mathbf{p}_{i}^{T} \mathbf{p}_{j}+\left(\mathbf{p}_{i}^{\perp}\right)^{T} \mathbf{p}_{j}^{\perp}
$$

em que ambos os produtos internos no lado direito são não nulos. De (3.32), nota-se que $\left\|\mathbf{p}_{\ell}^{\perp}\right\|<\sqrt{\varepsilon_{G S}}, \forall \ell$. Assim, usando a desigualdade de Cauchy-Schwarz (MEYER, 2000), obtémse $\left(\mathbf{p}_{i}^{\perp}\right)^{T} \mathbf{p}_{j}^{\perp}<\varepsilon_{G S}$. Como $\mathbf{p}_{i}^{T} \mathbf{p}_{j}=\widetilde{\mathbf{p}}_{i}^{T} \widetilde{\mathbf{p}}_{j}$, tem-se

$$
\left|\varphi\left(\mathbf{u}_{i}\right)^{T} \boldsymbol{\varphi}\left(\mathbf{u}_{j}\right)-\widetilde{\mathbf{p}}_{i}^{T} \widetilde{\mathbf{p}}_{j}\right|<\varepsilon_{G S},
$$

o que indica que o erro por usar a aproximação $\widetilde{\mathbf{p}}_{i}^{T} \widetilde{\mathbf{p}}_{j}$ nessa técnica é limitado pelo limiar $\varepsilon_{G S}$.

Para testar essa afirmação, uma simulação foi realizada onde 100 pontos de duas dimensões foram gerados aleatoriamente com uma distribuição normal com média zero em ambas as dimensões e desvio padrão unitário. A partir desses pontos, a matriz gramiano utilizando esses pontos foi calculada tanto usando o truque do kernel, quanto usando os produtos internos aproximados pelas projeções. Dessa forma, são obtidas todos os produtos internos entre os vetores combinados dois a dois. Para calcular os componentes das projeções foi usado o procedimento descrito na Seção 3.3 e os vetores para a base foram selecionados usando o procedimento descrito na Seção 3.5 utilizando $\varepsilon_{G S}=0,01$. A Figura 16 mostra o histograma das diferenças entre os produtos internos calculados pelo truque do kernel e pelo método proposto. É possível notar que o erro se concentra próximo ao zero e cai rapidamente se afastando dele. Também é 
possível verificar que o limite estabelecido na desigualdade (3.32) é respeitado.

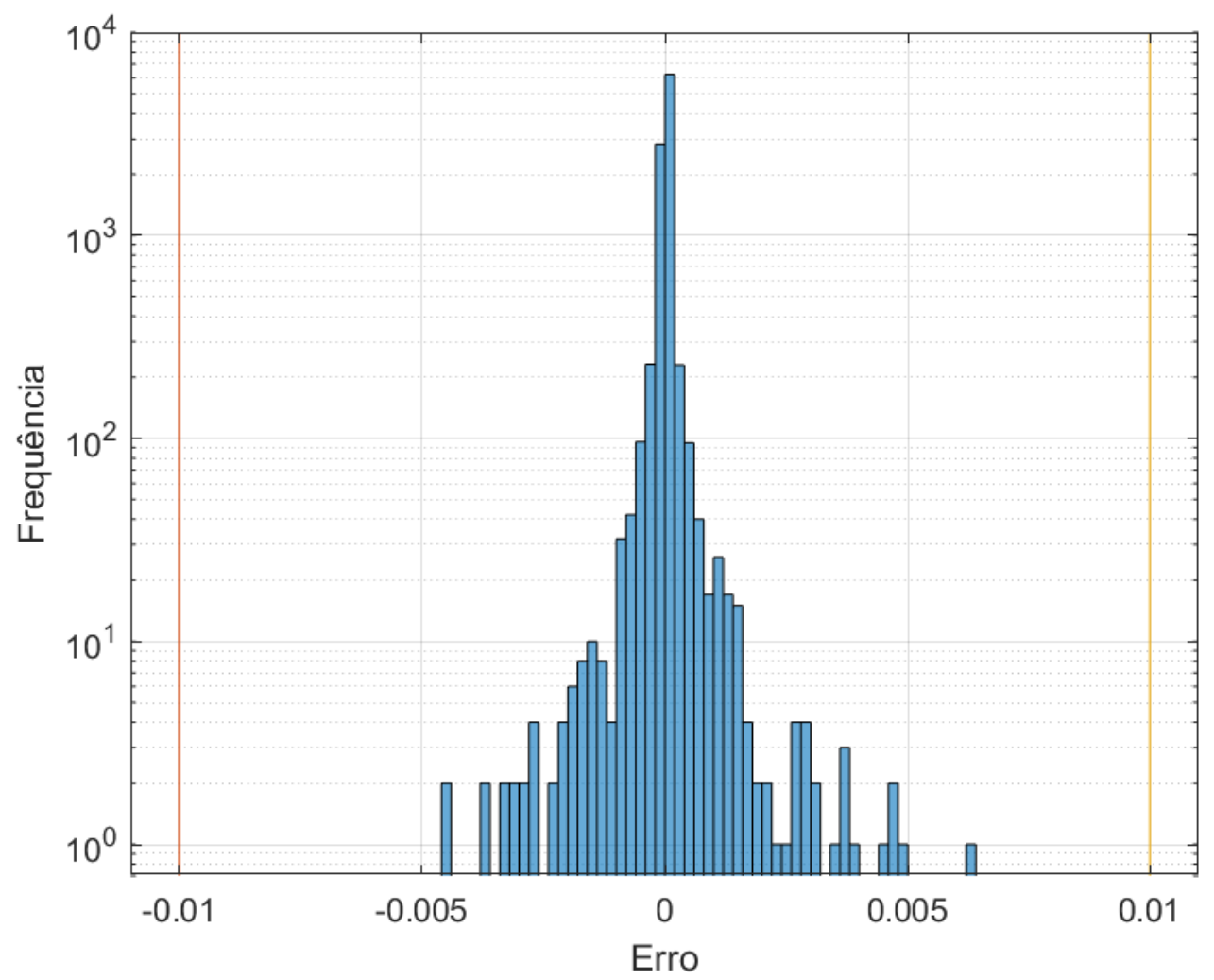

Figura 16: Histograma do erro nos produtos internos calculados usando o método proposto.

Fonte: Autor.

\subsection{Resultados de simulação: pré-imagem}

A pré-imagem de um conjunto em relação a uma função é o subconjunto pertencente ao domínio cuja imagem é o conjunto desejado (SCHOLKOPF; SMOLA, 2002). Com essa definição e a desigualdade (3.32), é possível calcular a pré-imagem do espaço $\mathcal{B}$, denotada por $\varphi^{-1}(\mathcal{B})$. A pré-imagem neste caso mostra a região do domínio onde os vetores de entrada são representados no espaço $\mathcal{B}$. Para exemplificar, foram realizados dois experimentos numéricos. Em ambos, o domínio é a região do $\mathbb{R}^{2}$ dada por $\mathcal{U}=[0,1] \times[0,1]$. Foram sorteados 500 pontos com distribuição uniforme nesse domínio e então o processo descrito nas Seções 3.3 e 3.5 foi utilizado. A base foi inicializada considerando-se aleatoriamente um desses 500 pontos. A pré-imagem foi calculada varrendo-se os pontos do domínio e verificando se eles atendiam o critério da Equação (3.32) para esse vetor. No passo seguinte, um novo vetor aleatório não pertencente à pré-imagem calculada anteriormente é inserido na base e uma nova pré-imagem é calculada. Esse processo se repete até que a região $[0,1] \times[0,1]$ consiga ser representada pelos 
vetores da base. A Figura 17 mostra o resultado para os oito primeiros vetores da base utilizando o kernel gaussiano com parâmetro $\zeta=1$. Já a Figura 18 mostra o resultado para os seis primeiros vetores da base utilizando-se o kernel polinomial de ordem 2. Em ambos os casos, $\varepsilon_{G S}=0,01$. Os vetores do dicionário estão circulados em vermelho e a região em verde mostra a área que será mapeada no espaço vetorial $\mathcal{B}$. É possível verificar por essas figuras que quanto mais vetores a base contiver, maior a região onde os vetores de entrada mapeiam vetores no espaço $\mathcal{B}$. Com apenas oito vetores no caso do kernel gaussiano e seis vetores no caso do kernel polinomial é possível abranger aproximadamente todo espaço de entrada. Além disso, no caso do kernel polinomial, quando o número de elementos na base atinge o valor da dimensão do espaço induzido pelo kernel, todo o $\mathbb{R}^{2}$ seria abrangido.

\subsection{Conclusão}

Neste capítulo foi apresentada uma técnica de esparsificação de dicionários para métodos kernel baseada no processo de ortogonalização de Gram-Schmidt. Esse processo se baseia em projeções dos vetores mapeados pelo kernel em um espaço de dimensão finita, mesmo quando o espaço induzido pelo kernel tem dimensão infinita. Mostrou-se que é possível representar essas projeções com um vetor de dimensão finita e que é possível aplicar o processo de GramSchmidt para calcular os componentes das projeções, mesmo sem ter explicitamente os vetores da base. Para selecionar os vetores que entram na base, verifica-se se a norma da componente ortogonal ao espaço $\mathcal{B}$ está acima de um limiar e, aplicando essa técnica, é possível obter uma aproximação dos produtos internos em $\mathcal{H}$ a partir do produto interno das projeções. Quando isso é feito, o erro máximo para essa aproximação é o limiar de comparação como mostra o histograma na Figura 16. Conforme se inserem vetores na base, a região do espaço de entrada que mapeia vetores cuja projeção em $\mathcal{B}$ é uma boa aproximação aumenta, como mostrado nas Figuras 17 e 18. Por fim, cabe observar que apesar de se adotar um caminho bem distinto do considerado em (ENGEL; MANNOR; MEIR, 2004), chegou-se aqui ao mesmo critério de esparsificação do ALD. No entanto, no próximo capítulo, a técnica de esparsificação aqui proposta é aplicada ao KLMS, sendo vantajosa em termos de custo computacional em comparação com o ALD-KLMS. 


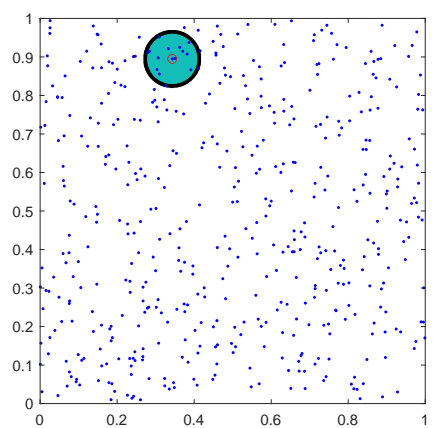

(a) $\mathrm{N}=1$

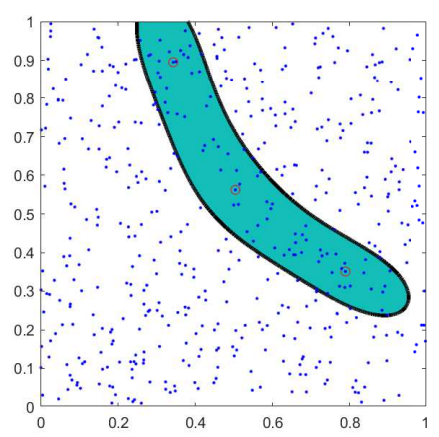

(c) $\mathrm{N}=3$

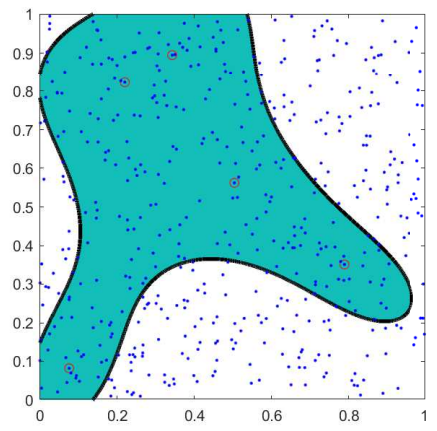

(e) $\mathrm{N}=5$

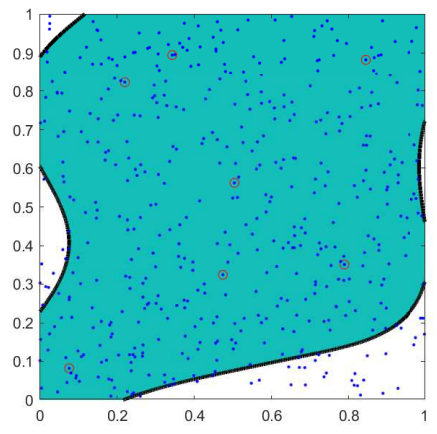

(g) $\mathrm{N}=7$

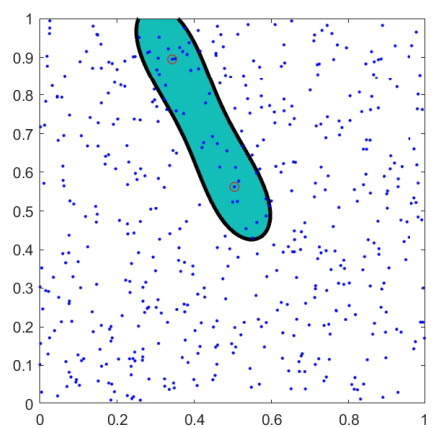

(b) $\mathrm{N}=2$

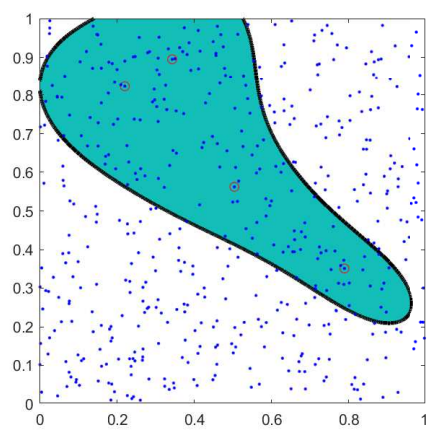

(d) $\mathrm{N}=4$

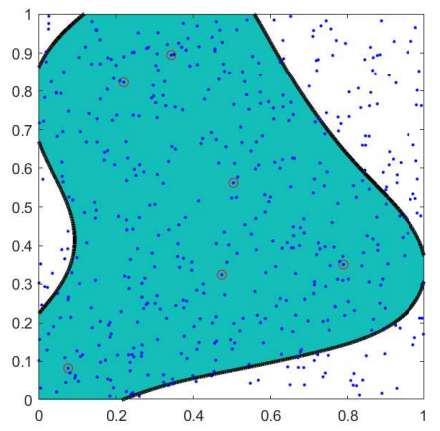

(f) $\mathrm{N}=6$

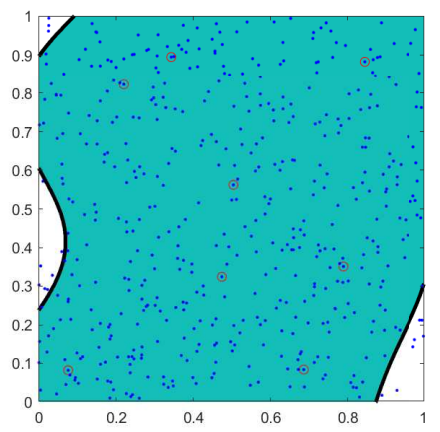

(h) $\mathrm{N}=8$

Figura 17: Evolução da pré-imagem para kernel gaussiano Fonte: Autor. 


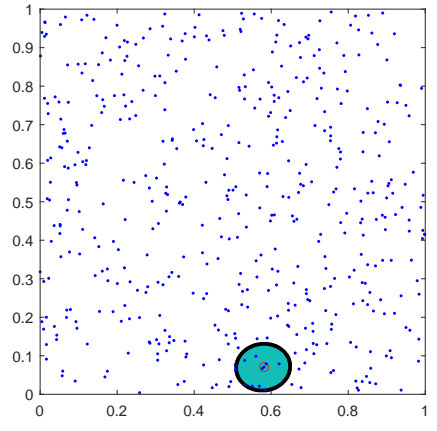

(a) $\mathrm{N}=1$

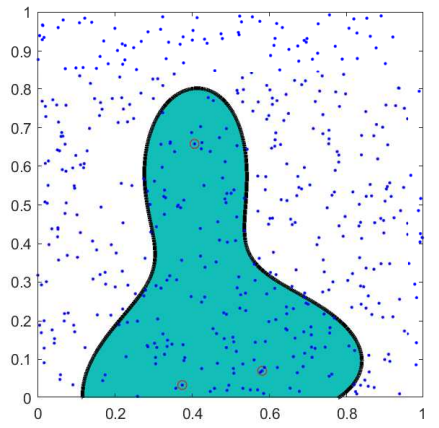

(c) $\mathrm{N}=3$

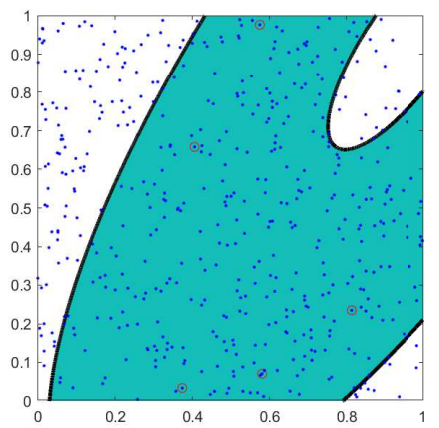

(e) $\mathrm{N}=5$

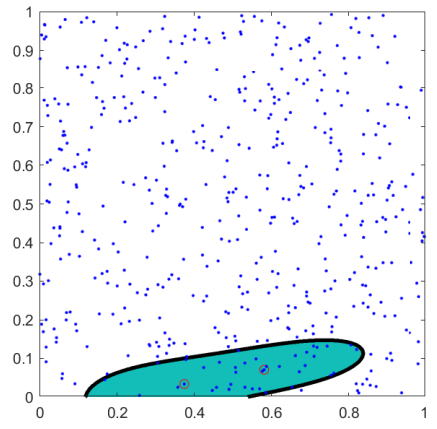

(b) $\mathrm{N}=2$

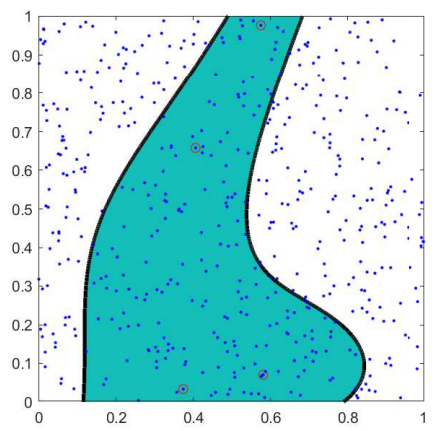

(d) $\mathrm{N}=4$

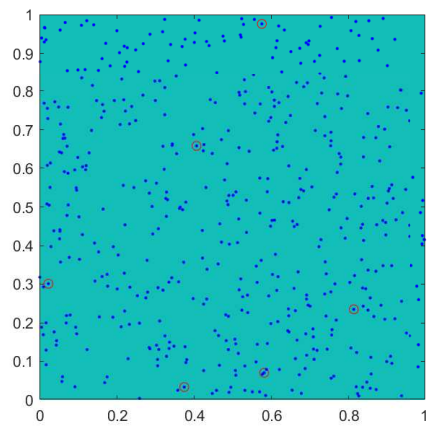

(f) $\mathrm{N}=6$

Figura 18: Evolução da pré-imagem para kernel polinomial Fonte: Autor. 


\section{APLICAÇÃO EM FILTRAGEM ADAPTATIVA}

Neste capítulo, a técnica de esparsificação proposta é aplicada ao algoritmo KLMS. Em seguida, uma forma de retirar elementos do dicionário dinamicamente é proposta. Ao longo do capítulo, apresentam-se simulações com o intuito de mostrar a efetiva funcionalidade do algoritmo proposto.

\subsection{Aplicando a esparsificação proposta no KLMS: o algoritmo Gram-Schmidt KLMS}

A ideia do algoritmo proposto nesta dissertação é substituir o vetor mapeado $\varphi(u(n))$ por sua projeção no espaço $\mathcal{B}$, denotada por $\mathbf{p}(n)^{1}$. Assim, substituindo $\varphi(u(n))$ nas Equações (1.4) a (1.6) da página 3 , obtém-se

$$
\begin{aligned}
y(n) & =\mathbf{p}^{T}(n) \omega(n-1), \\
e(n) & =d(n)-y(n), \\
\omega(n) & =\omega(n-1)+\mu e(n) \mathbf{p}(n),
\end{aligned}
$$

em que $\omega(0)=\mathbf{0}$. Como ocorre com o KLMS descrito pelas Equações (1.4) a (1.6), o algoritmo descrito por (4.1) a (4.3) não pode ser implementado em um computador digital. Assim como $\boldsymbol{\varphi}(\mathbf{u}(n))$, sua projeção $\mathbf{p}(n)$ no espaço $\mathcal{B}$ pode ter dimensão infinita, o que ocorre quando se considera o kernel gaussiano, por exemplo.

Definindo $a(n)=\mu e(n)$ como no KLMS, (4.3) pode ser reescrita como

$$
\omega(n)=\sum_{\ell=1}^{n} a(\ell) \mathbf{p}(\ell) .
$$

Observa-se então que $\omega(n)$ é uma combinação linear dos vetores $\mathbf{p}(j) \in \mathcal{B}, j=1,2, \cdots, n$. Como foi visto no Capítulo 3, esses vetores podem ser escritos como uma combinação linear dos vetores da base $\mathbf{B}_{N(n)}$ denotados por $\left\{\boldsymbol{\beta}_{1}, \boldsymbol{\beta}_{2}, \cdots, \boldsymbol{\beta}_{N(n)}\right\}$. Assim,

$$
\mathbf{p}(n)=\sum_{j=1}^{N(n)} p_{j}(n) \boldsymbol{\beta}_{j}
$$

Consequentemente, $\omega(n)$ também pode ser escrito como uma combinação linear dos vetores da

\footnotetext{
${ }^{1}$ Neste capítulo, não se utiliza subescrito no vetor $\mathbf{p}$ como no Capítulo 3. Assim, a projeção do vetor $\varphi(u(n))$ é denotada por $\mathbf{p}(n)$.
} 
base $\mathbf{B}_{N(n)}$, ou seja,

$$
\boldsymbol{\omega}(n)=\sum_{i=1}^{N(n)} w_{i}(n) \boldsymbol{\beta}_{i}
$$

em que $w_{i}(n), i=1,2, \cdots N(n)$ são escalares. Substituindo (4.5) e (4.6) em (4.1), chega-se a

$$
y(n)=\mathbf{p}^{T}(n) \boldsymbol{\omega}(n-1)=\sum_{i=1}^{N(n)} \sum_{j=1}^{N(n)} w_{i}(n) p_{j}(n)\left\langle\boldsymbol{\beta}_{i}, \boldsymbol{\beta}_{j}\right\rangle .
$$

Usando o procedimento descrito no Capítulo 3, o vetor $\mathbf{p}(n)$ pode ser substituído por sua representação de dimensão finita

$$
\widetilde{\mathbf{p}}(n)=\mathbf{H}_{N(n)} \boldsymbol{\kappa}_{N(n)}(\mathbf{u}(n)) \text {. }
$$

Definindo o vetor

$$
\mathbf{w}(n)=\left[w_{1}(n) w_{2}(n) \cdots w_{N(n)}(n)\right],
$$

e usando (4.8), a saída do filtro, dada por (4.7), pode ser reescrita como o produto interno dos vetores $\widetilde{\mathbf{p}}(n)$ e $\mathbf{w}(n)$, ambos de dimensão $N(n)$, o que leva a

$$
y(n)=\widetilde{\mathbf{p}}^{T}(n) \mathbf{w}(n-1) .
$$

Assim, as Equações (4.1)-(4.3) podem ser reescritas como

$$
\begin{aligned}
y(n) & =\widetilde{\mathbf{p}}^{T}(n) \mathbf{w}(n-1), \\
e(n) & =d(n)-y(n), \\
\mathbf{w}(n) & =\mathbf{w}(n-1)+\mu e(n) \widetilde{\mathbf{p}}(n) .
\end{aligned}
$$

Diferente do KLMS convencional, ao se usar $\widetilde{\mathbf{p}}(n)$ como entrada, $\mathbf{w}(n)$ passa a ter a dimensão do dicionário, ou seja, $N(n)$. Neste caso, como o vetor $\widetilde{\mathbf{p}}(n)$ foi calculado, é natural associar ao algoritmo a técnica de esparsificação do Capítulo 3. O algoritmo resultante é denotado por GS-KLMS (Gram-Schmidt KLMS) e suas equações são mostradas na Tabela 7. Nessa tabela, para simplificar a notação, os subscritos dos vetores e matrizes foram suprimidos. Além disso, a cardinalidade do dicionário foi denotada por $N$ em vez de $N(n)$, mas cabe salientar que o valor de $N$ aumenta de uma unidade quando um novo vetor de entrada é inserido em $\mathcal{D}$. Quando isso acontece, a dimensão do vetor de coeficientes do filtro também aumenta e um zero é inserido na $(N+1)$-ésima posição do vetor. 
Tabela 7: Sumário do algoritmo GS-KLMS

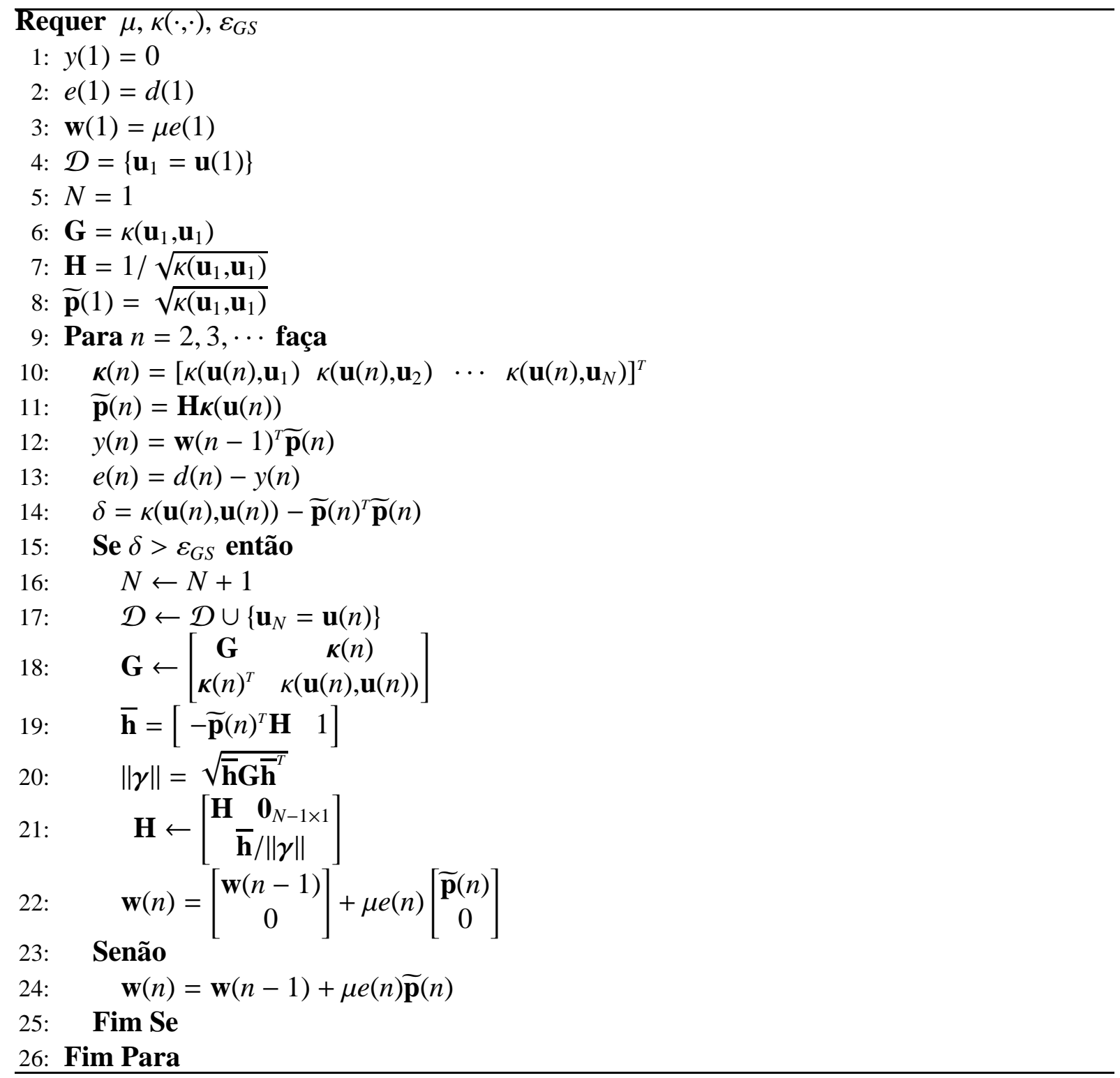

Fonte: Autor.

\subsubsection{Resultados de Simulação}

Os resultados de simulação estão organizados em subseções, levando em conta a aplicação e a função kernel considerada.

\subsubsection{Predição não-linear com kernel gaussiano}

Revisitando mais uma vez o exemplo do Capítulo 1 da página 5, foi incorporado agora o resultado para o GS-KLMS. Os parâmetros, como passo de adaptação e os limiares, continuam os mesmos. O limiar $\varepsilon_{G S}$ foi escolhido igual ao limiar $\varepsilon_{A L D}$ para comparação. O MSE é 
mostrado na Figura 19 e o tamanho do dicionário na Figura 20. É possível verificar que o desempenho do GS-KLMS é similar ao dos outros algoritmos, especificamente é mais próximo ao do ALD-KLMS. Em termos de dicionário, o GS-KLMS e o ALD-KLMS apresentam o mesmo número de elementos. Isso se dá, pois o critério de esparsificação é o mesmo nos dois algoritmos. No entanto, o ALD-KLMS não considera uma base ortonormal como o GS-KLMS. Essa diferença faz com que esses algoritmos tenham custos computacionais distintos, como pode ser observado nas curvas de número de operações de multiplicação acumuladas e número de operações não lineares acumuladas, mostradas respectivamente nas Figuras 21 e 22. Até a iteração $n=10^{4}$, o GS-KLMS realiza 9,6 $\times 10^{7}$ multiplicações acumuladas, enquanto o ALD-KLMS realiza $3,3 \times 10^{8}$. Além disso, o número de operações não lineares acumuladas realizadas até $n=10^{4}$ pelo GS-KLMS é $1,1 \times 10^{6}$, praticamente o mesmo que o do ALD-KLMS, tornando o GS-KLMS o algoritmo que menos realiza operações entre os apresentados.

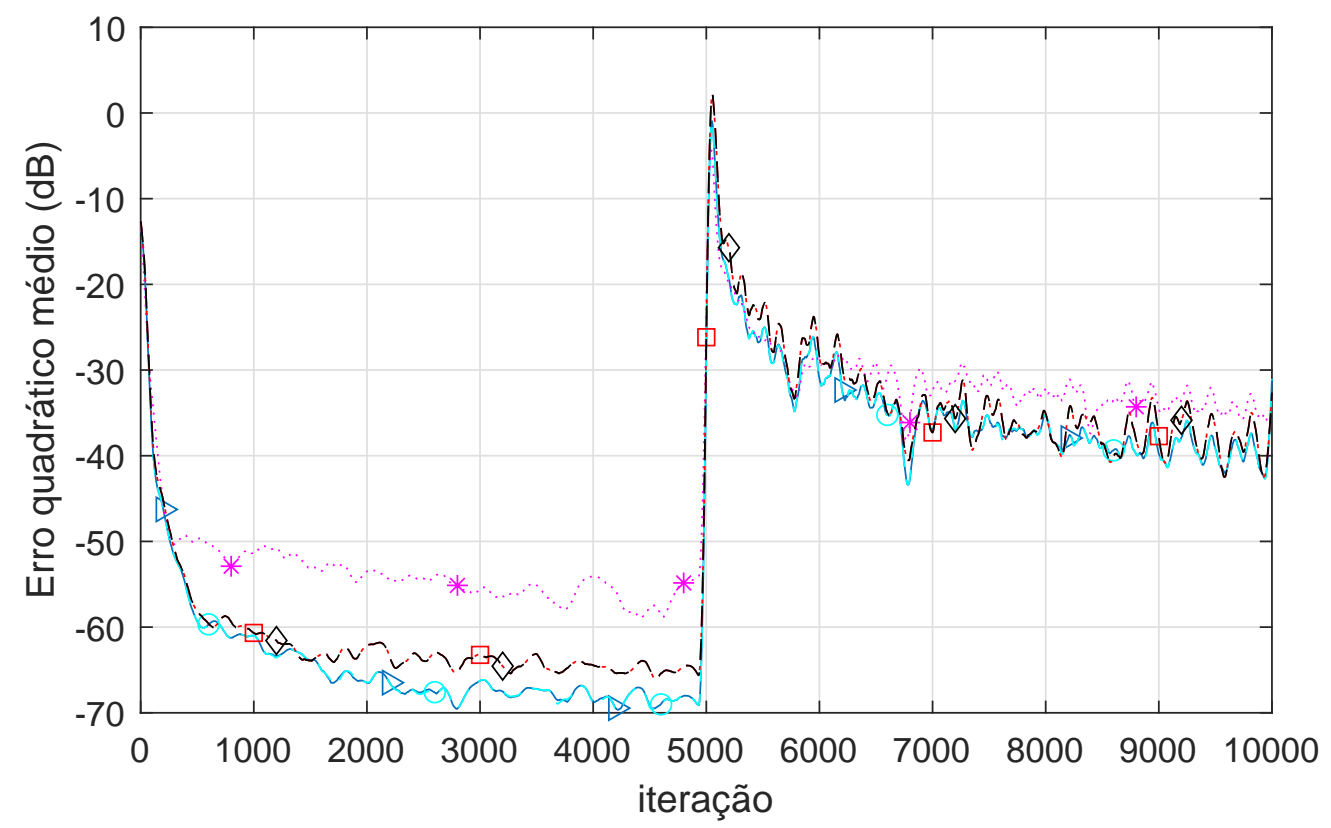

\begin{tabular}{|c|c|}
\hline $\begin{array}{c}\Rightarrow \text { KLMS } \\
-\exists-\cdot \text { ALD-KLM }\end{array}$ & $\begin{array}{l}-\ominus-\text { QKLMS } \\
-\ominus-- \text { GS-KLMS }\end{array}$ \\
\hline
\end{tabular}

Figura 19: MSE para o problema de predição não linear considerando kernel gaussiano, $\zeta=1$, $M=7, \mu=0,5, \varepsilon_{Q}=0,01, \varepsilon_{G S}=\varepsilon_{A L D}=0,0199$ e $D=5000$.

Fonte: Autor 


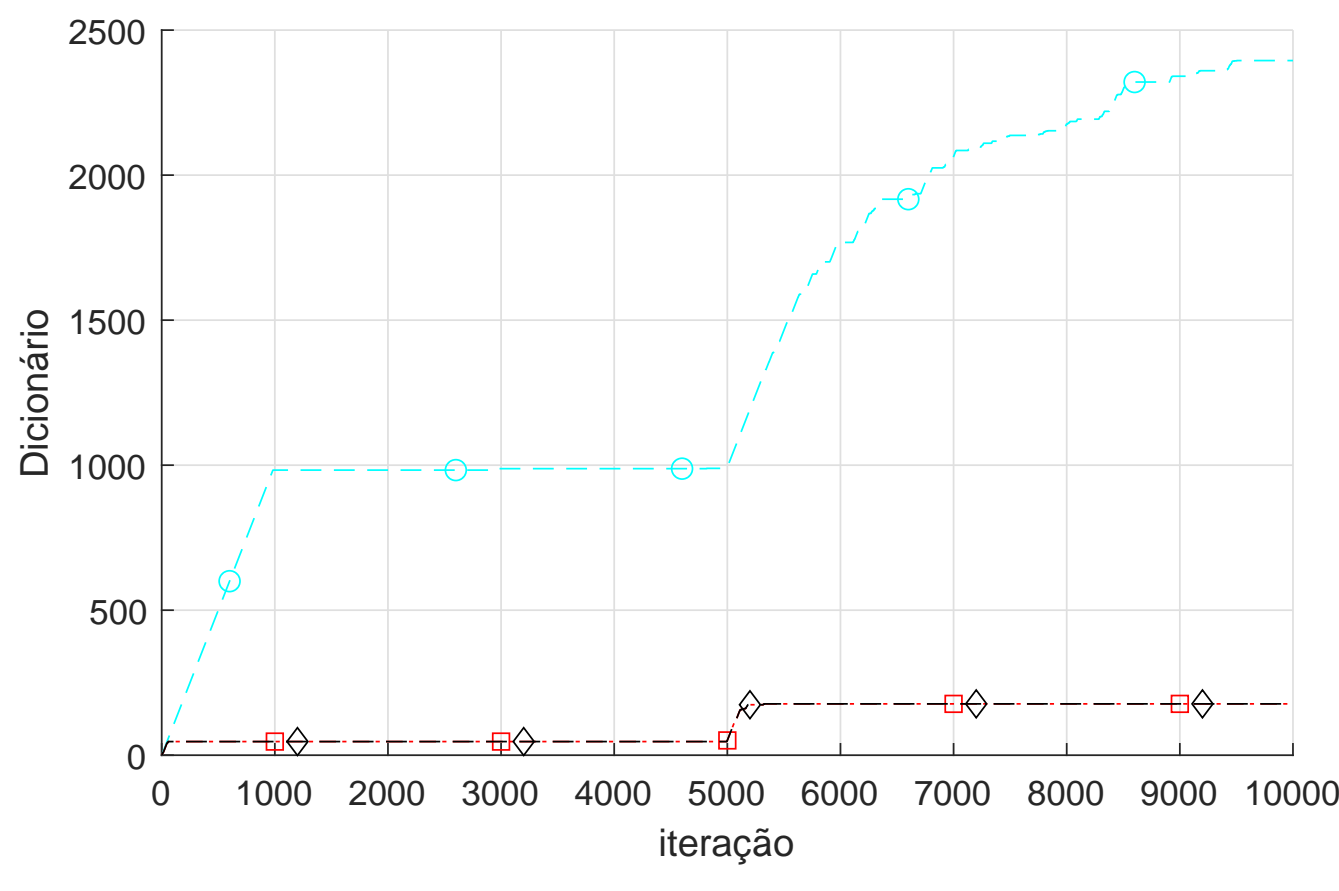

$-\ominus-\cdot$ QKLMS $-\cdot \boxminus-\cdot \cdot$ ALD-KLMS $-\diamond-\cdot$ GS-KLMS

Figura 20: Tamanho do dicionário para o problema de predição não linear considerando kernel gaussiano, $\zeta=1, M=7, \mu=0,5, \varepsilon_{Q}=0,01, \varepsilon_{G S}=\varepsilon_{A L D}=0,0199$ e $D=5000$.

Fonte: Autor

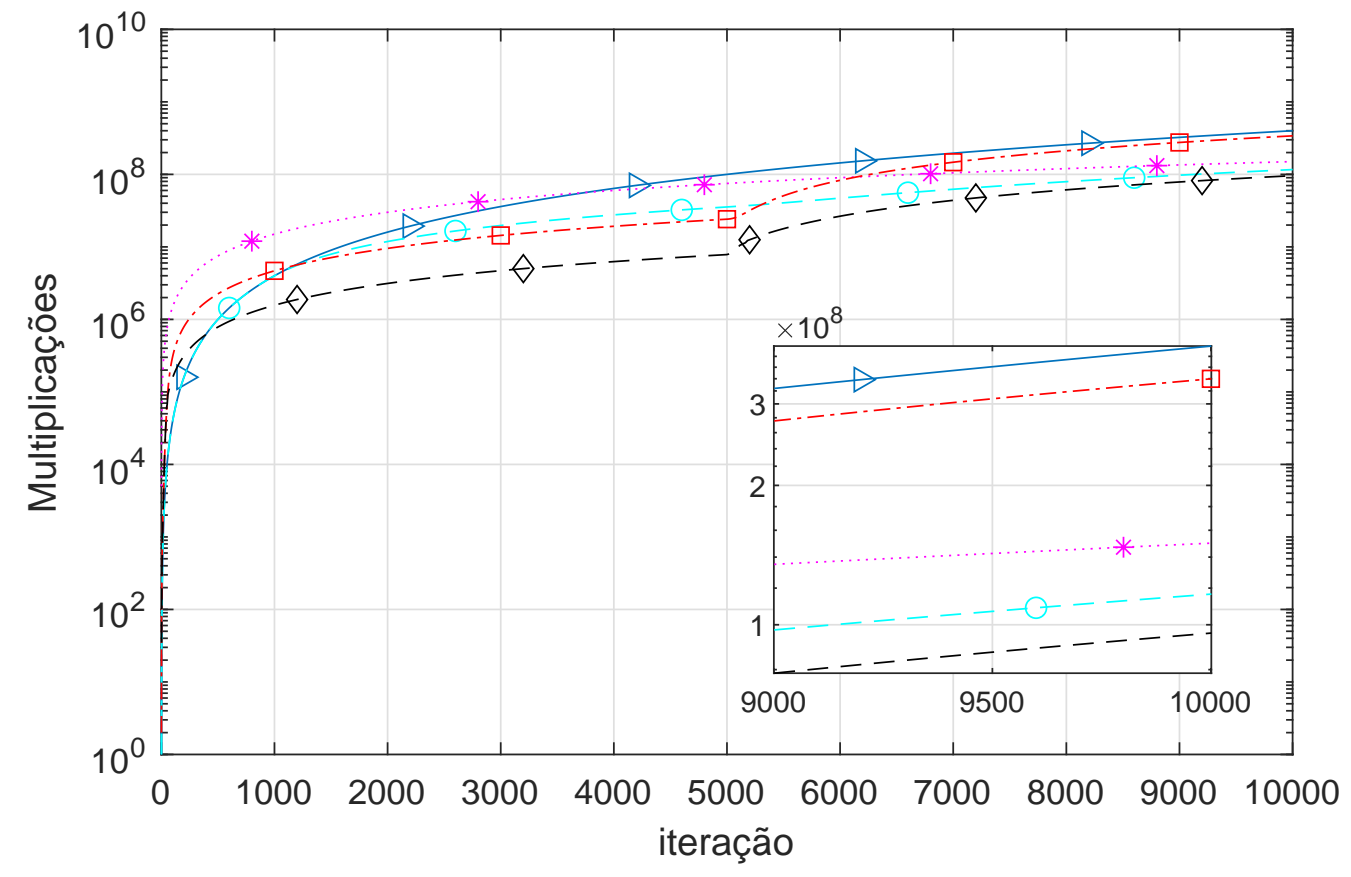

$\triangleright$ KLMS $-\ominus-$ QKLMS $\cdots \cdot \cdots \cdot * \cdots \cdot$ RFF-KLMS $-\cdot €-\cdot \cdot$ ALD-KLMS $-\oslash-\cdot$ GS-KLMS

Figura 21: Número acumulado de operações de multiplicações para o problema de predição não linear considerando kernel gaussiano, $\zeta=1, M=7, \mu=0,5, \varepsilon_{Q}=0,01, \varepsilon_{G S}=\varepsilon_{A L D}=0,0199$ e $D=5000$. 


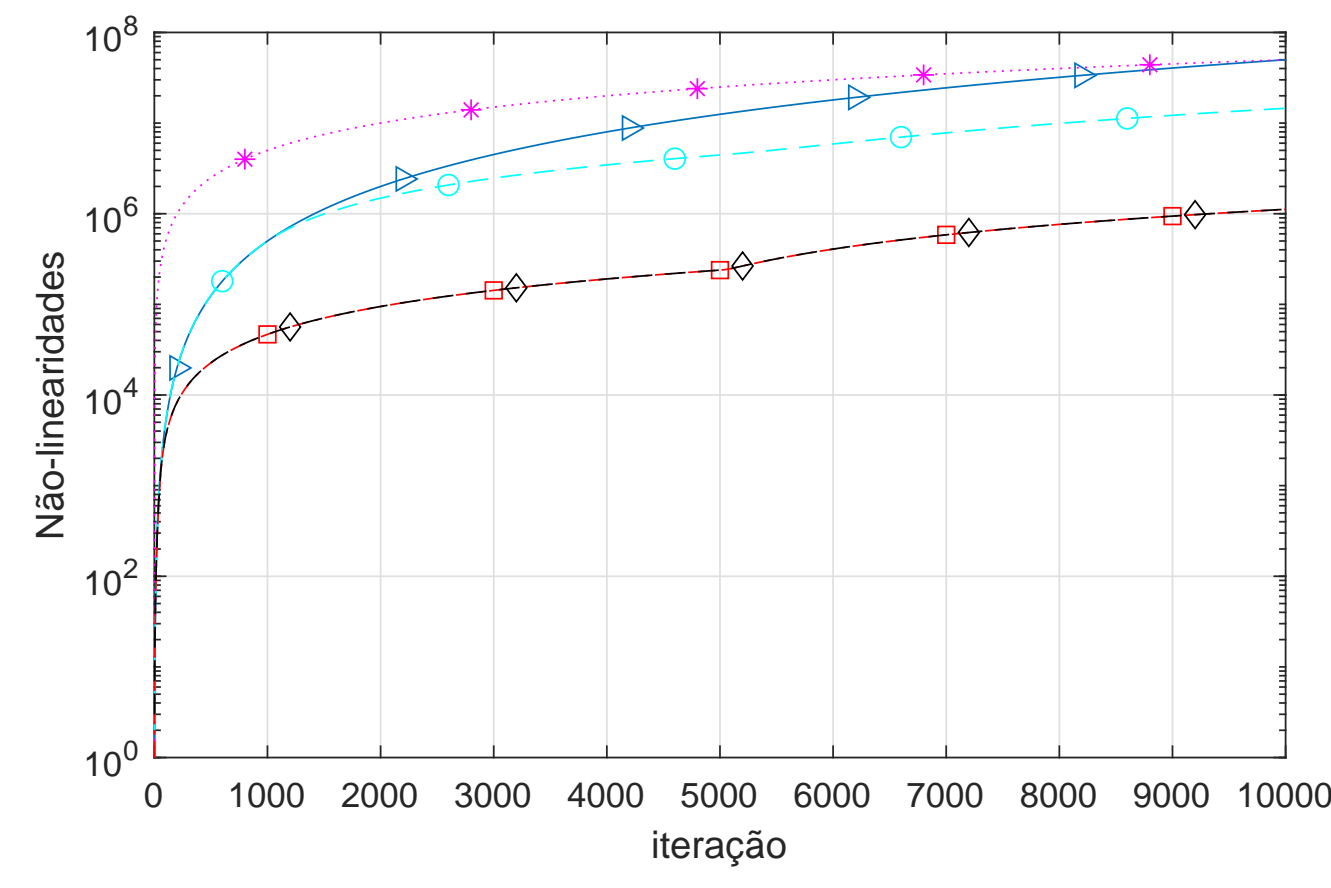

$\longrightarrow$ KLMS $-\ominus-$ QKLMS $\cdots \cdot \cdots \cdot * \cdots \cdot$ RFF-KLMS $-\cdot €-\cdot \cdot$ ALD-KLMS $-\oslash-\cdot$ GS-KLMS

Figura 22: Número acumulado de operações não lineares para o problema de predição não linear considerando kernel gaussiano, $\zeta=1, M=7, \mu=0,5, \varepsilon_{Q}=0,01, \varepsilon_{G S}=\varepsilon_{A L D}=0,0199$ e $D=5000$.

Fonte: Autor

O mesmo exemplo foi simulado considerando-se um ruído somado ao sinal gerado pela Equação (1.8) da página 5 sem considerar a mudança abrupta. Foram consideradas relações sinal-ruído de 10, 20, 30, 40, 50 e 60dB. A Figura 23 mostra o desempenho em termos de MSE para os algoritmos KLMS, GS-KLMS, QKLMS e RFF-KLMS. É possível perceber que quanto menor a relação sinal-ruído, maior o erro de predição e que para relações sinal-ruído muito altas a curva de MSE fica parecida com o exemplo sem ruído (mostrado na figura como relação sinal-ruído infinita). A Figura 24 mostra que o tamanho do dicionário para o GS-KLMS e para o QKLMS não varia com o ruído sendo que o GS-KLMS apresenta em regime menos de 50 vetores no dicionário enquanto o QKLMS necessita de cerca de 1000 vetores. Pode-se observar que os algoritmos apresentam aproximadamente o mesmo comportamento em termos de MSE independentemente da relação sinal-ruído. Isso era esperado já que os parâmetros de adaptação foram ajustados para que isso ocorresse. Além disso, o ruído causa um pequeno aumento na velocidade de convergência dos algoritmos. No caso do RFF com $S N R=60 \mathrm{~dB}$, nota-se que o MSE é menor que o caso sem ruído, o que será investigado em um trabalho futuro. 


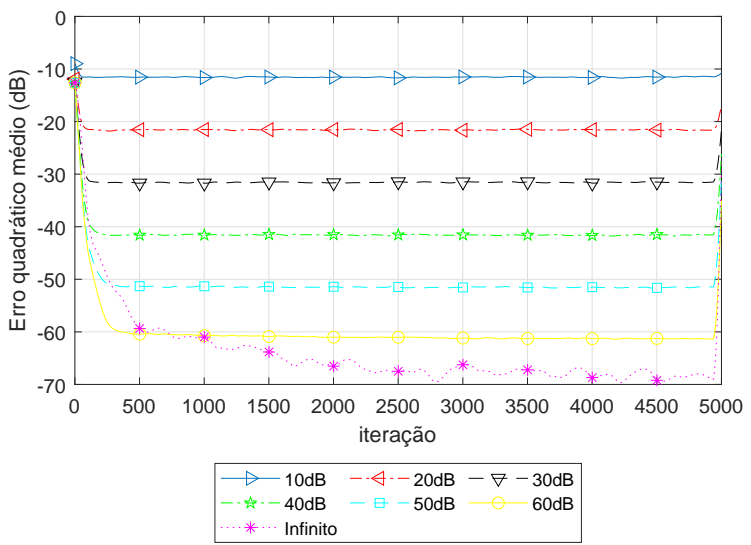

(a) KLMS

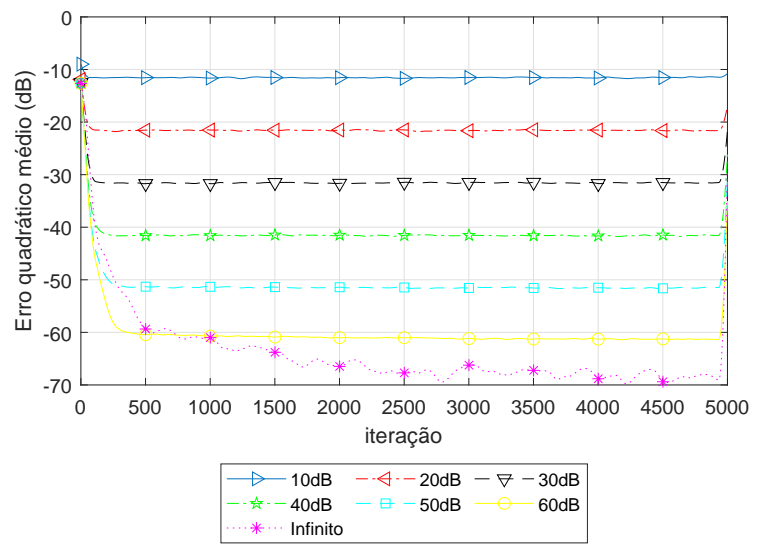

(c) QKLMS

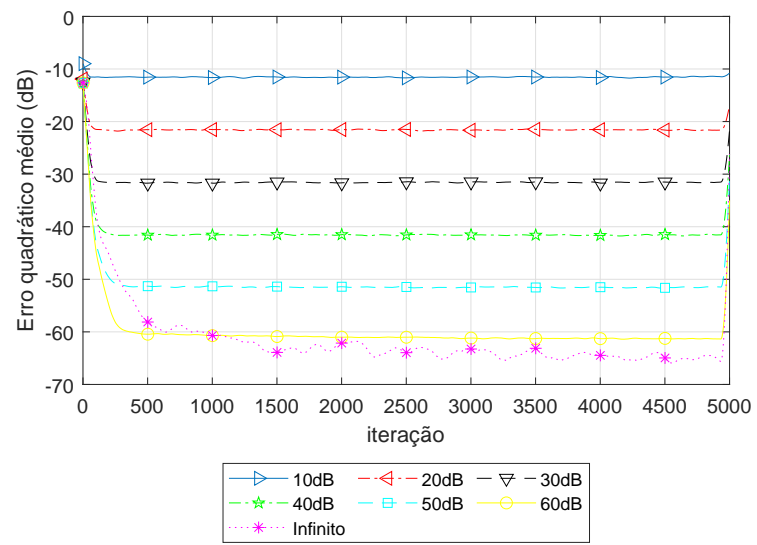

(b) GS-KLMS

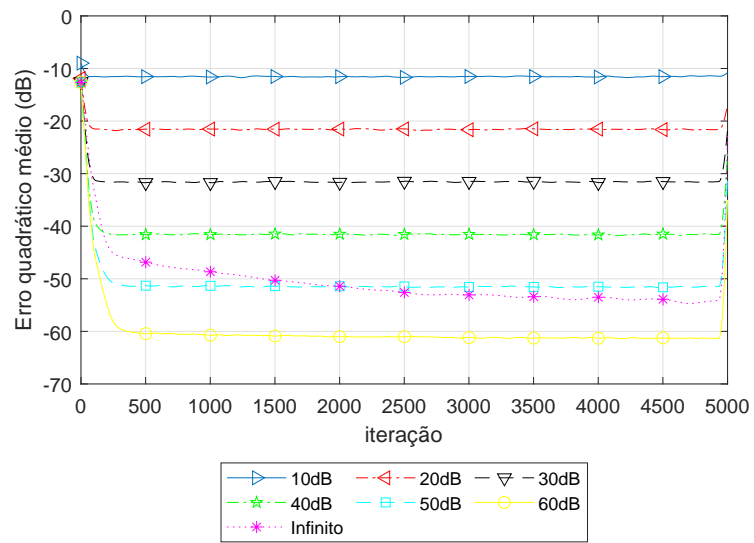

(d) RFF-KLMS

Figura 23: MSE para o problema de predição não linear considerando kernel gaussiano, $\zeta=1$, $M=7, \mu=0,5, \varepsilon_{Q}=0,01, \varepsilon_{G S}=0,0199$ e $D=5000$ e ruído com potência variando conforme indicado; média de 100 realizações.

Fonte: Autor.

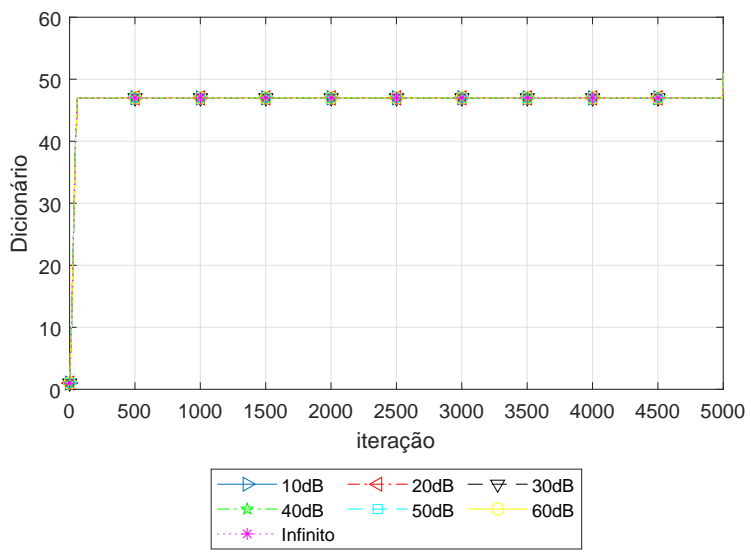

(a) GS-KLMS

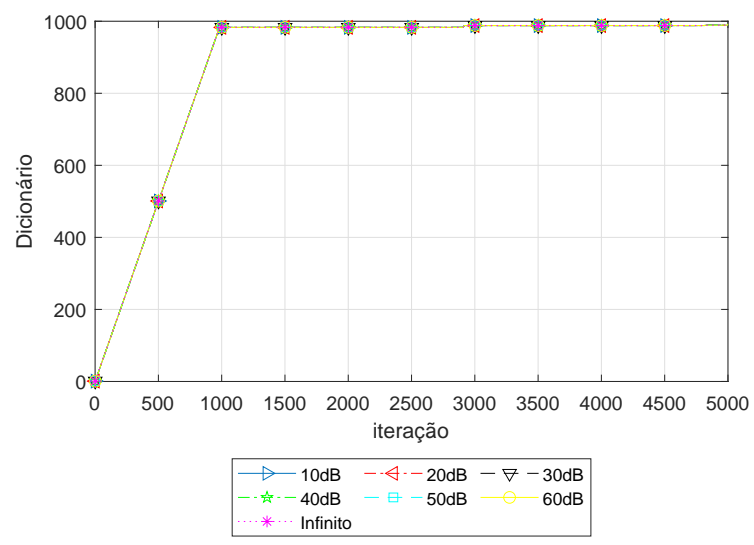

(b) QKLMS

Figura 24: Tamanho do dicionário para o problema de predição não linear considerando kernel gaussiano, $\zeta=1, M=7, \mu=0,5, \varepsilon_{Q}=0,01, \varepsilon_{G S}=0,0199$ e ruído com potência variando conforme indicado; média de 100 realizações.

Fonte: Autor. 


\subsubsection{Predição não-linear com kernel polinomial}

A seguinte simulação utiliza novamente as mesmas equações do exemplo do Capítulo 1 da página 5 , porém com outros parâmetros. São consideradas $3 \times 10^{4}$ iterações e a mudança abrupta ocorre em $n=1,5 \times 10^{4}$. O kernel utilizado nesta simulação é o polinomial de ordem 2 e considera-se $M=7$. O passo de adaptação é $10^{-4}$ e $\varepsilon_{Q}=\varepsilon_{A L D}=\varepsilon_{G S}=0,1$. O RFF-KLMS, o NC-KLMS e o CC-KLMS não foram considerados nessa comparação. O RFF-KLMS por não ser possível utiliza-lo com kernel polinomial e o NC-KLMS e CC-KLMS por não atingirem resultados comparáveis aos dos demais algoritmos. É possível verificar que, para obter o mesmo MSE, como mostrado na Figura 25, o QKLMS precisou de um dicionário aproximadamente 7 vezes maior que o do GS-KLMS, realizando praticamente o dobro de multiplicações acumuladas até $n=3 \times 10^{4}$ e 10 vezes o número de operações não lineares até a mesma iteração em relação ao GS-KLMS. Já o ALD-KLMS precisou do mesmo número de vetores no dicionário que o GS-KLMS, realizou o mesmo número de operações não lineares até a iteração $n=3 \times 10^{4}$ mas precisou de aproximadamente $2 \times 10^{7}$ multiplicações acumuladas até a última iteração a mais que o GS-KLMS.

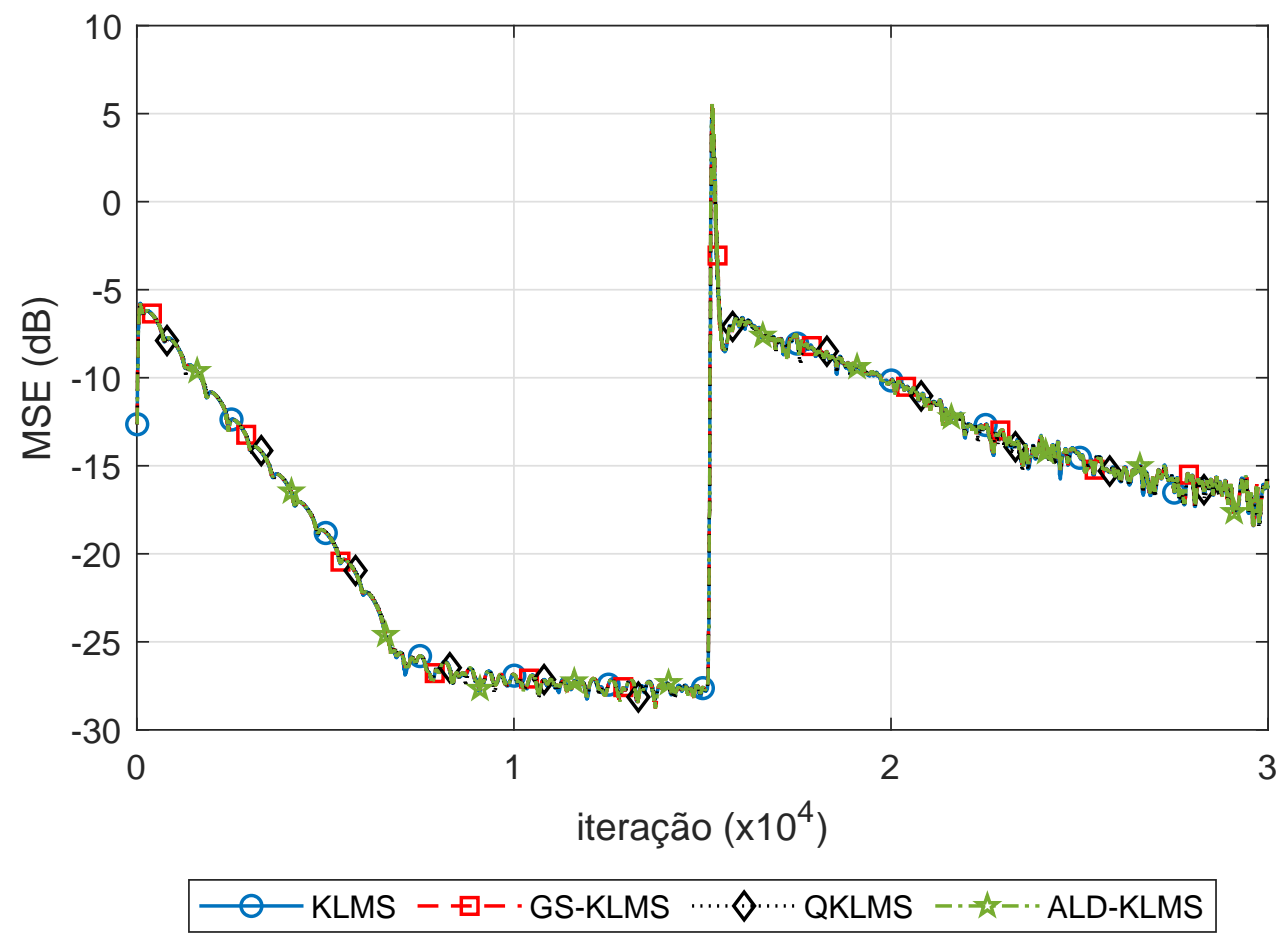

Figura 25: MSE para o problema de predição não linear considerando kernel polinomial, $q=2$, $M=7, \mu=10^{-4}, \varepsilon_{Q}=\varepsilon_{G S}=\varepsilon_{A L D}=0,1$.

Fonte: Autor. 


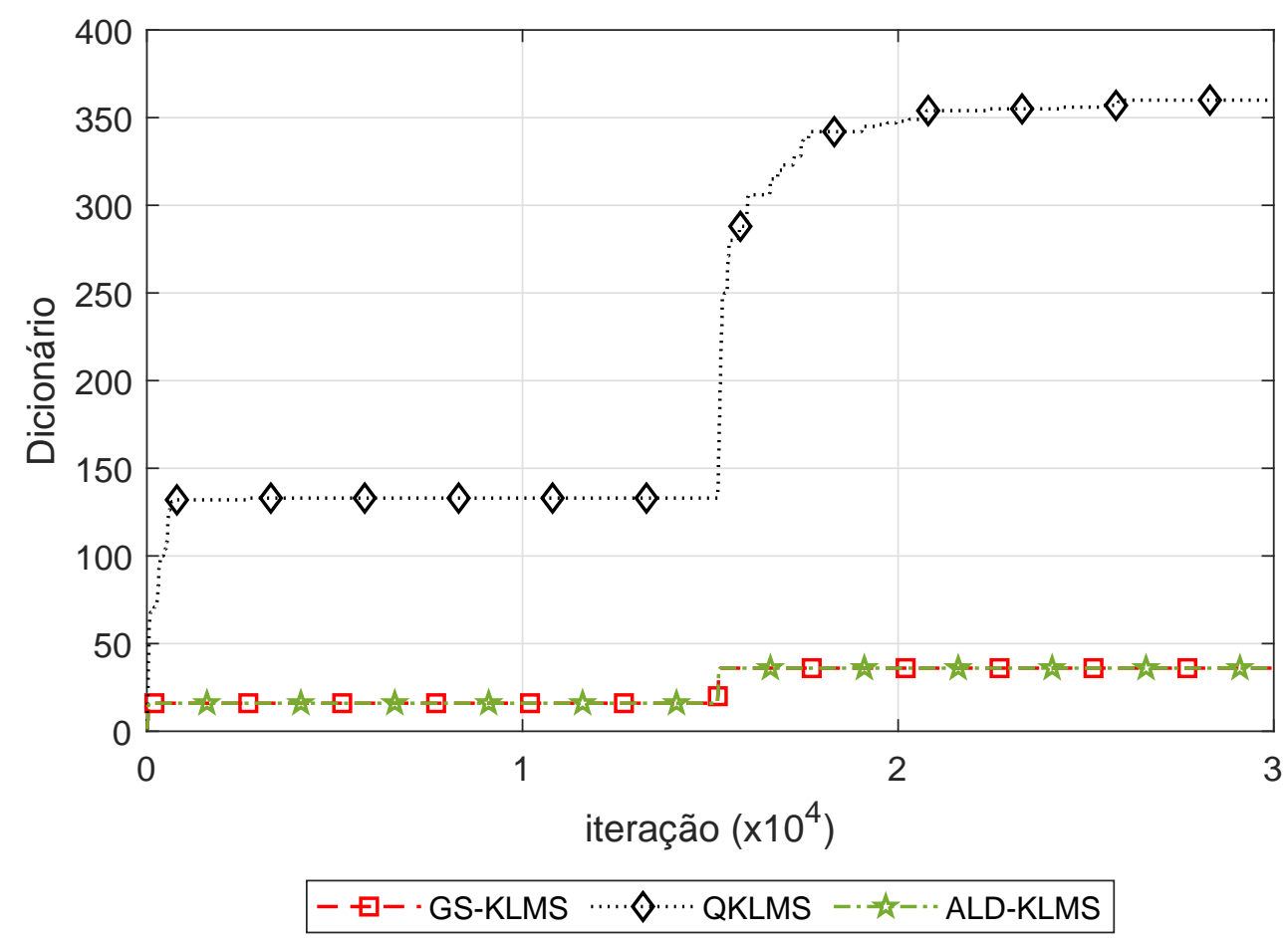

Figura 26: Tamanho do dicionário no problema de predição não linear considerando kernel polinomial, $q=2, M=7, \mu=10^{-4}, \varepsilon_{Q}=\varepsilon_{G S}=\varepsilon_{A L D}=0,1$.

Fonte: Autor.

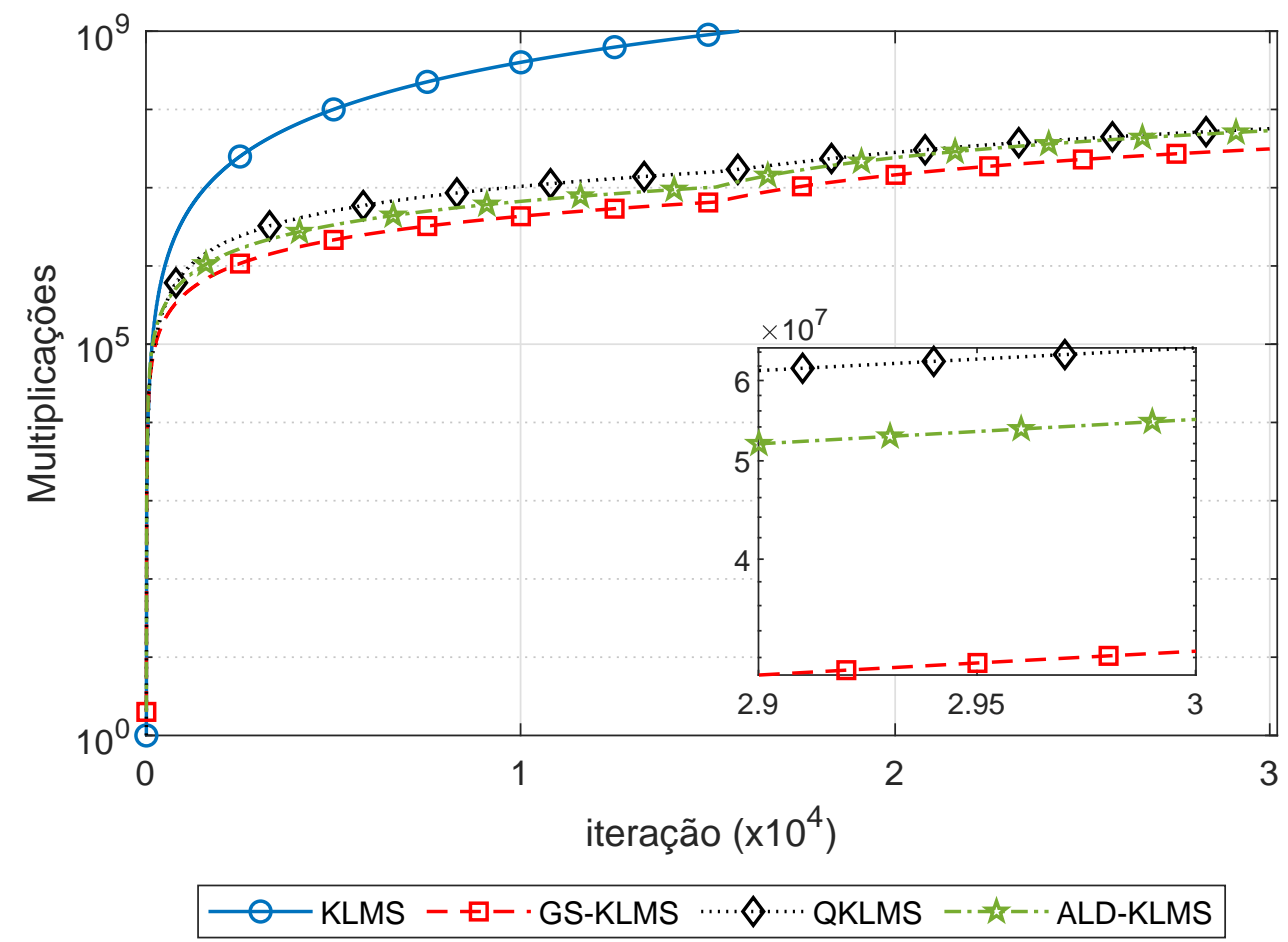

Figura 27: Número de multiplicações acumuladas para o problema de predição não linear considerando kernel polinomial, $q=2, M=7, \mu=10^{-4}, \varepsilon_{Q}=\varepsilon_{G S}=\varepsilon_{A L D}=0,1$.

Fonte: Autor. 


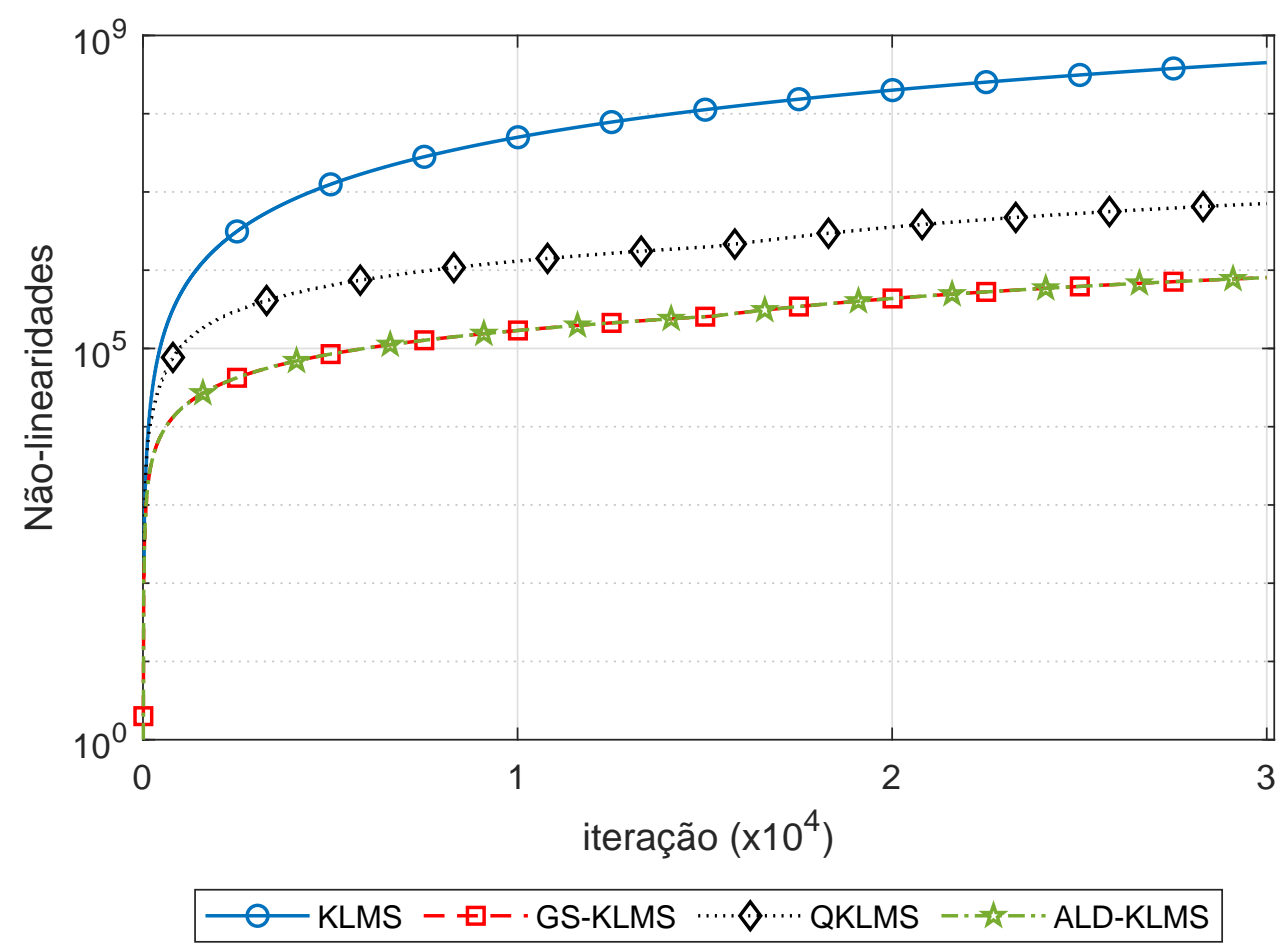

Figura 28: Número de operações não lineares acumuladas para o problema de predição não linear considerando kernel polinomial, $q=2, M=7, \mu=10^{-4}, \varepsilon_{Q}=\varepsilon_{G S}=\varepsilon_{A L D}=0,1$.

Fonte: Autor.

\subsubsection{Predição de série temporal de manchas solares}

Nesta simulação é considerada a série temporal de manchas solares de janeiro de 1749 a setembro de 2016 que pode ser obtida de (HATHAWAY, D., 2017). Foi considerado o kernel gaussiano com $\zeta=10, \mathbf{u}(n)=[u(n-1) u(n-2) u(n-3)]^{T}$ e horizonte de predição de uma amostra (SAIDÉ,C.; LENGELLÉ; HONEINE; RICHARD; ACHKAR, 2015). Os algoritmos mostraram desempenho em termos de MSE similares como mostra a Figura 29. O tamanho dos dicionários do ALD-KLMS e do GS-KLMS ao final do experimento foi de 11 vetores enquanto que o dicionário do QKLMS obteve 96 vetores, como apresentado na Figura 30. O RFF-KLMS não foi apresentado nessa figura pois não armazena um dicionário explicitamente. O número de operações de multiplicação acumuladas realizadas pelo QKLMS é aproximadamente $3 \times 10^{5}$ maior que o número dessas operações realizadas pelo GS-KLMS, como visto na Figura 31. Já o número de operações não lineares acumuladas realizadas pelo QKLMS, apresentado na Figura 32, ao final do experimento é de $2 \times 10^{5}$ enquanto que o mesmo número para o GS-KLMS é $4,2 \times 10^{4}$. A série original e a série predita pelo GS-KLMS são apresentadas na Figura 33 . 


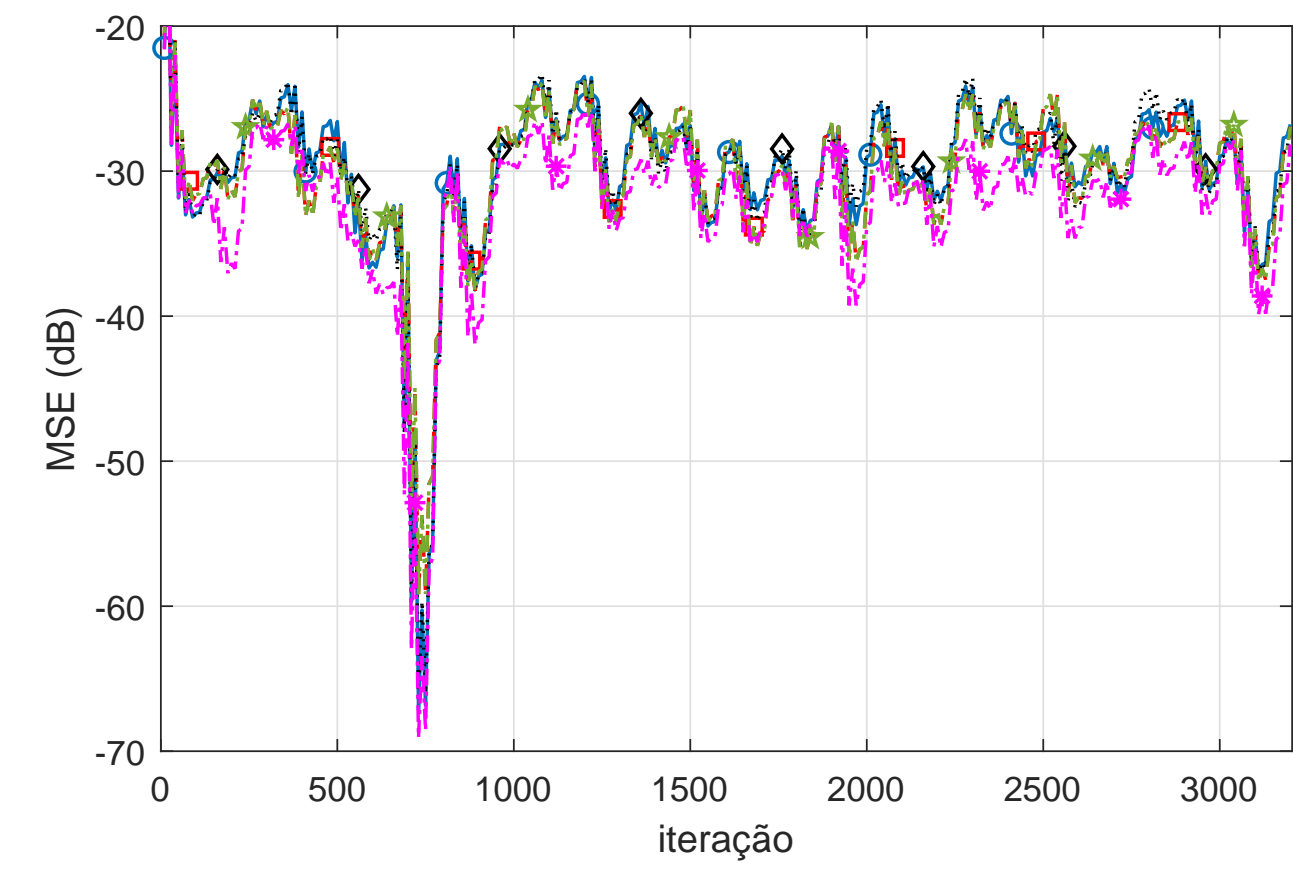

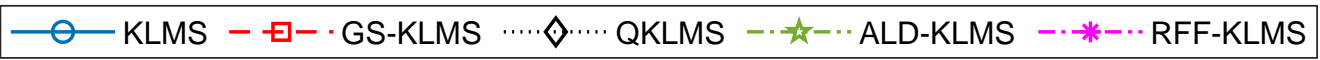

Figura 29: MSE no problema de predição não linear de manchas solares para kernel gaussiano com parâmetro $\zeta=10, \mu=1,0, M=3$, para $\varepsilon_{G S}=0,75, \varepsilon_{A L D}=0,75, \varepsilon_{Q}=0,1$ e $D=1000$.

Fonte: Autor

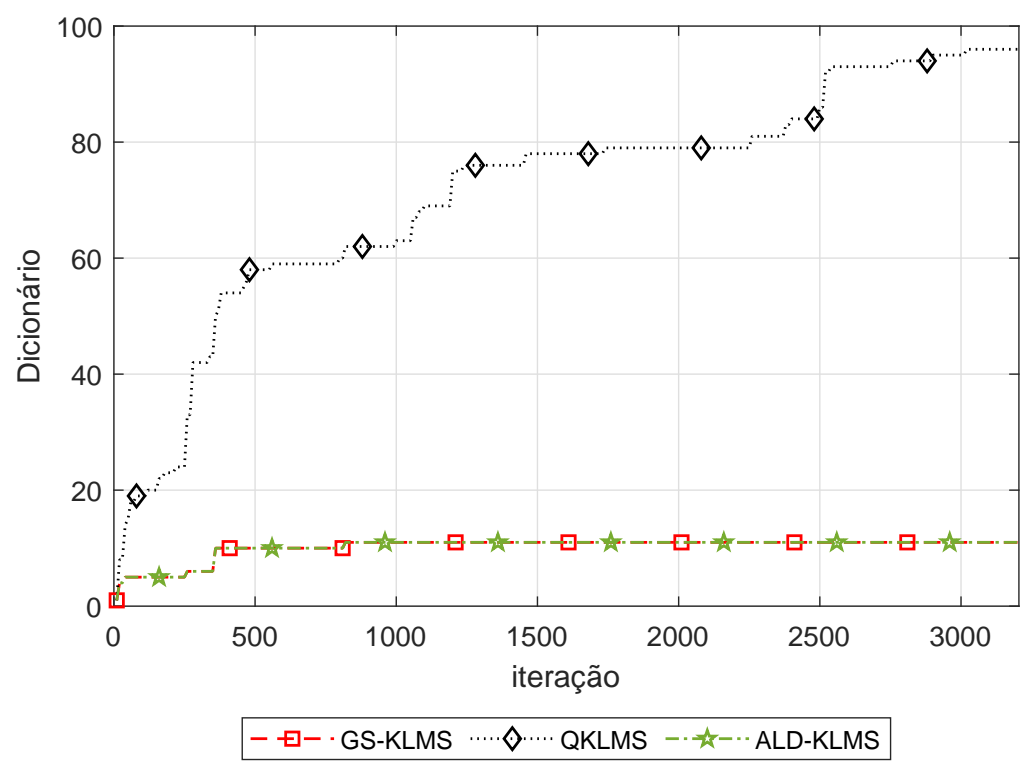

Figura 30: Tamanho do dicionário no problema de predição não linear de manchas solares para kernel gaussiano com parâmetro $\zeta=10, \mu=1,0, M=3$, para $\varepsilon_{G S}=0,75, \varepsilon_{A L D}=0,75$, $\varepsilon_{Q}=0,1$ e $D=1000$.

Fonte: Autor 


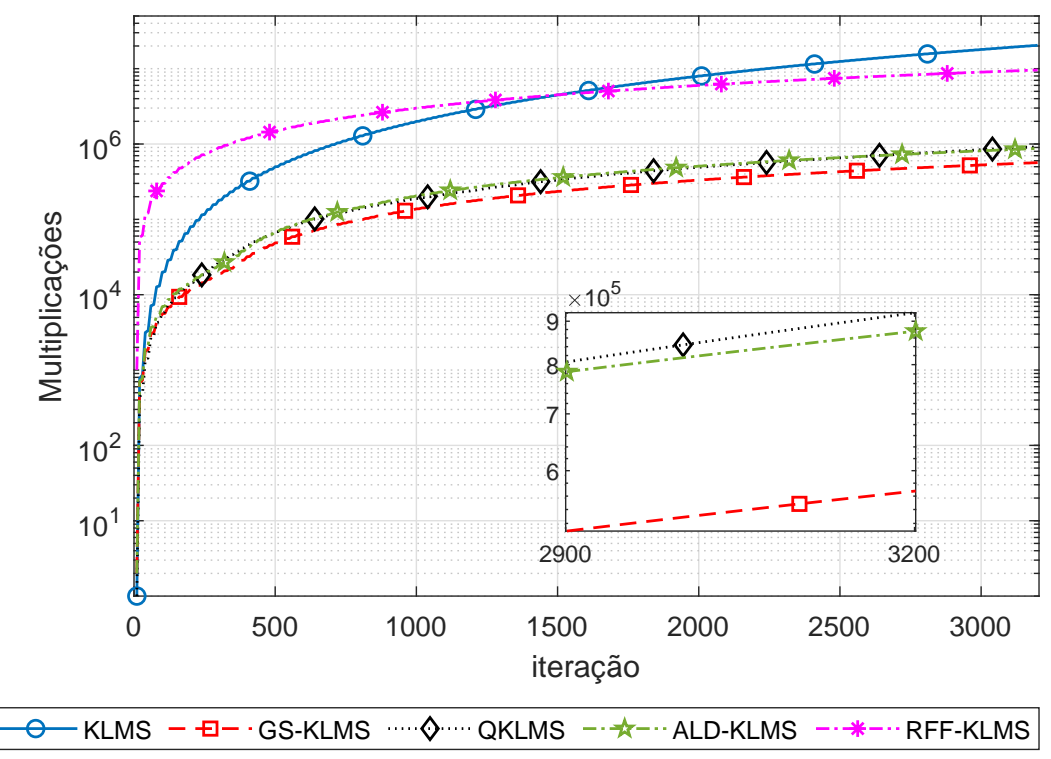

Figura 31: Número de operações de multiplicação no problema de predição não linear de manchas solares para kernel gaussiano com parâmetro $\zeta=10, \mu=1,0, M=3$, para $\varepsilon_{G S}=0,75$, $\varepsilon_{A L D}=0,75, \varepsilon_{Q}=0,1$ e $D=1000$.

Fonte: Autor

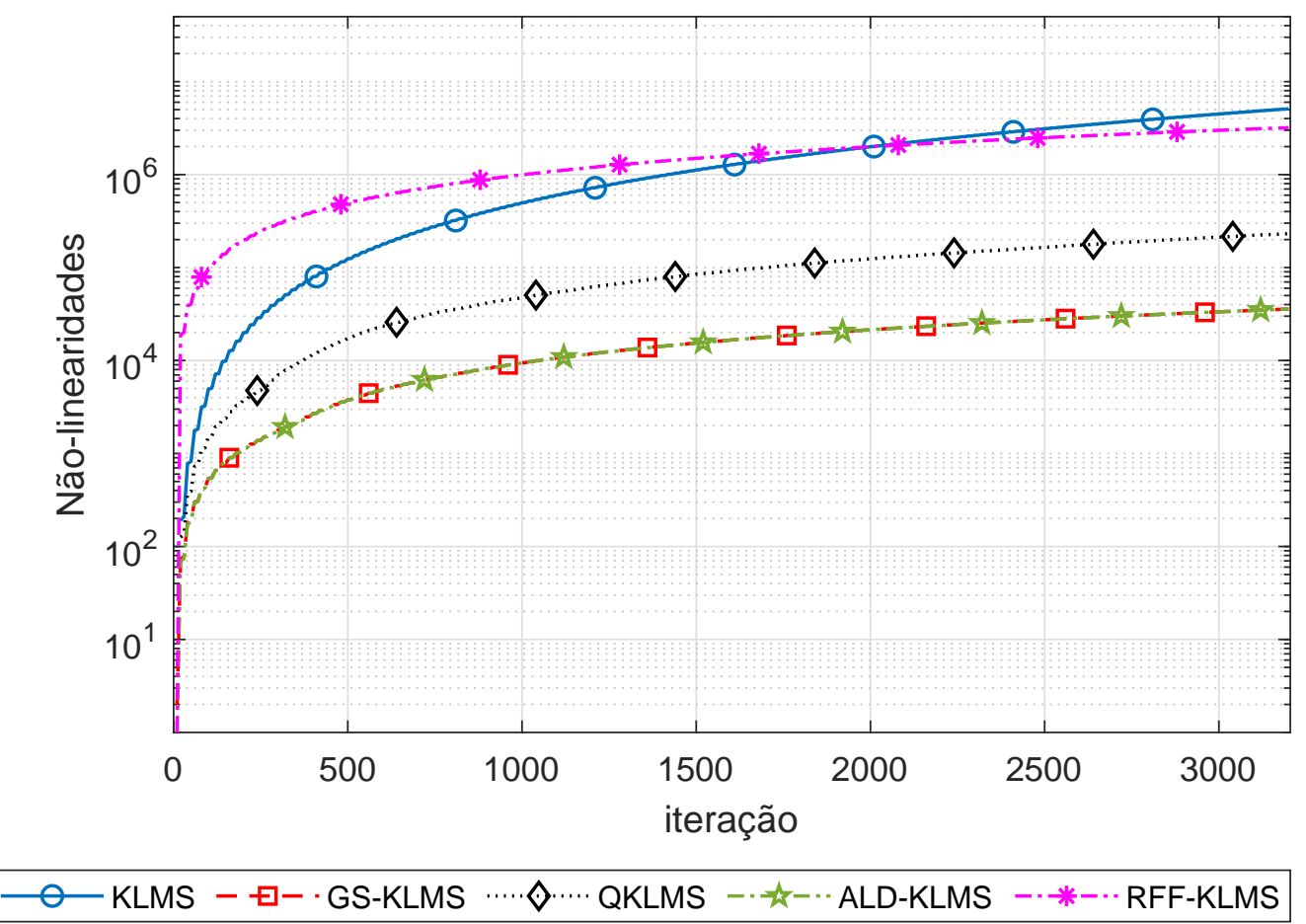

Figura 32: Número de operações não lineares no problema de predição não linear de manchas solares para kernel gaussiano com parâmetro $\zeta=10, \mu=1,0, M=3$, para $\varepsilon_{G S}=0,75$, $\varepsilon_{A L D}=0,75, \varepsilon_{Q}=0,1$ e $D=1000$. 


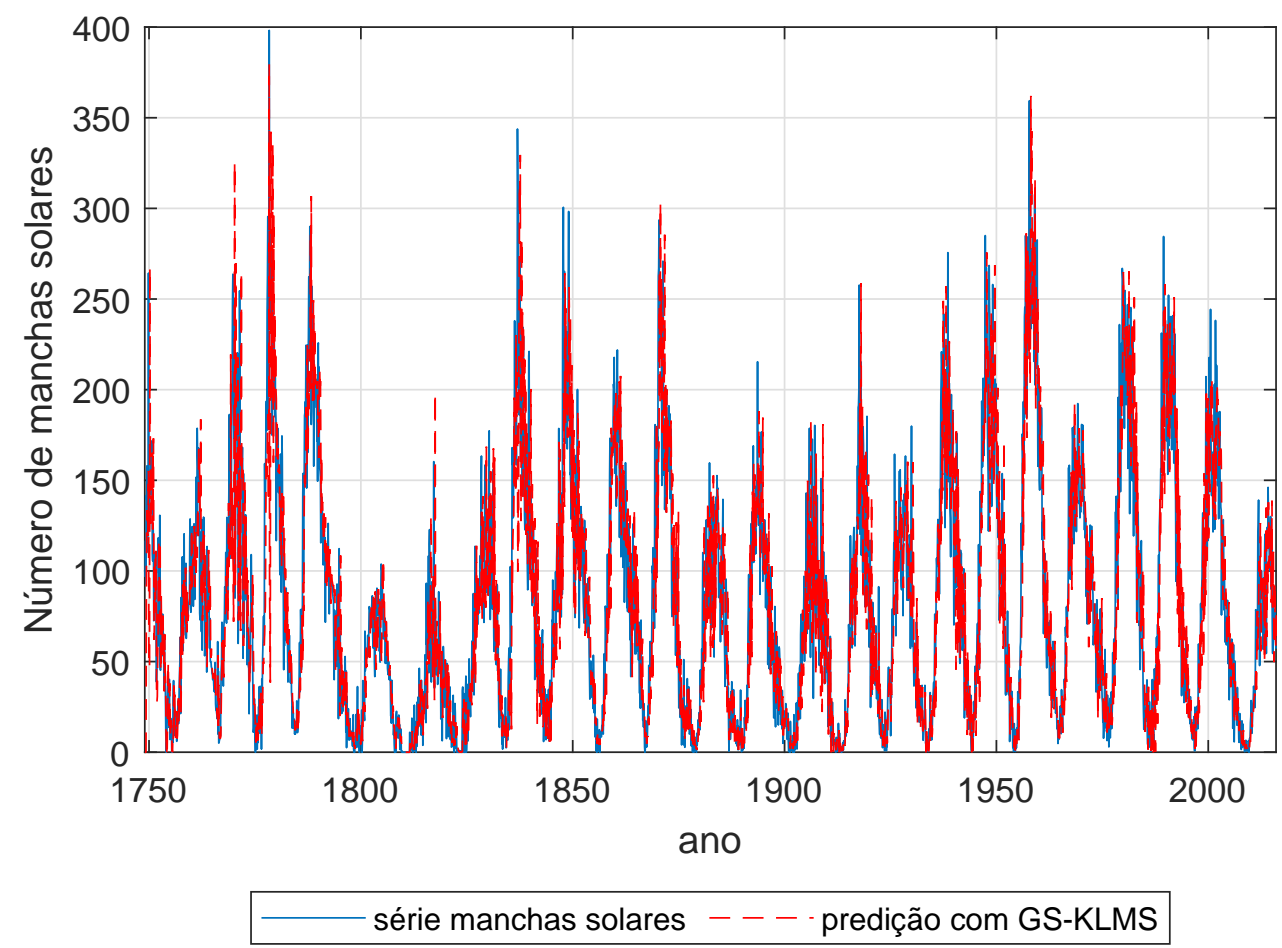

Figura 33: Série de manchas solares e série predita.

Fonte: Autor

\subsubsection{Equalização de canal não linear}

Os algoritmos também foram comparados em um cenário de simulação considerado o problema de equalização de canal de comunicação não linear. O canal contém uma parte linear dada por

$$
z(n)=u(n)+0,5 u(n-1),
$$

seguido por uma não linearidade tal que

$$
x(n)=z(n)-0,9 z^{2}(n)+v(n),
$$

em que $v(n)$ é um ruído branco gaussiano não correlacionado com a entrada, de média nula e desvio padrão 0,1 , o que leva a uma relação sinal ruído de $23 \mathrm{~dB}$. Na iteração $n=2,5 \times 10^{5}$ considera-se uma mudança abrupta no canal de modo que sua parte não linear se torna

$$
x(n)=z(n)+0,8 z^{2}(n)+v(n),
$$

em que $v(n)$ é mantido com as mesmas estatísticas da primeira metade da simulação. O sinal de entrada é binário, no conjunto $\{-1,1\}$, e o equalizador usa as últimas seis amostras do sinal 
de saída do canal como entrada, ou seja, $M=6$. O kernel usado é o polinomial com parâmetro $q=2$ e passo de adaptação 0,0001. Consideraram-se apenas os filtros KLMS, QKLMS, ALDKLMS e GS-KLMS. O NC-KLMS e o CC-KLMS não foram considerados devido ao baixo desempenho neste problema em comparação aos outros algoritmos. Já o RFF-KLMS não foi considerado porque o kernel polinomial não é invariante à translação. A Figura 34 mostra o desempenho em termos de MSE. Percebe-se que todos os filtros tiveram um desempenho parecido, chegando a um MSE de aproximadamente -11 dB. A Figura 35 mostra o tamanho do dicionário dos filtros QKLMS, ALD-KLMS e GS-KLMS. É possível notar que o dicionário do GS-KLMS e do ALD-KLMS têm o mesmo tamanho que corresponde aproximadamente a um quarto do tamanho do dicionário do QKLMS na primeira metade e um oitavo na segunda metade. As Figuras 36 e 37 mostram, respectivamente, o número acumulado de operações de multiplicação e o número acumulado de operações não lineares. É possível perceber que enquanto o número de não-linearidades dos algoritmos ALD-KLMS e GS-KLMS são iguais e corresponde a aproximadamente um quinto do número de não linearidades acumuladas do QKLMS. Já o número de multiplicações do GS-KLMS é $5,2 \times 10^{8}$, sendo novamente mais baixo que o número acumulado de multiplicações do QKLMS que é de 5,8 × 10 8 e o do ALDKLMS que é de $9,0 \times 10^{8}$. A Figura 38 mostra a saída do GS-KLMS que idealmente deveria ser -1 ou +1 . É possível observar que o filtro consegue equalizar o canal já que se pode ver uma separação dos símbolos. Apesar de se obter uma taxa de erro de bits de aproximadamente 0,2, que é considerada alta para transmissão binária (LATHI, 1998), cabe observar que um filtro linear não conseguiria equalizar o canal neste caso. 


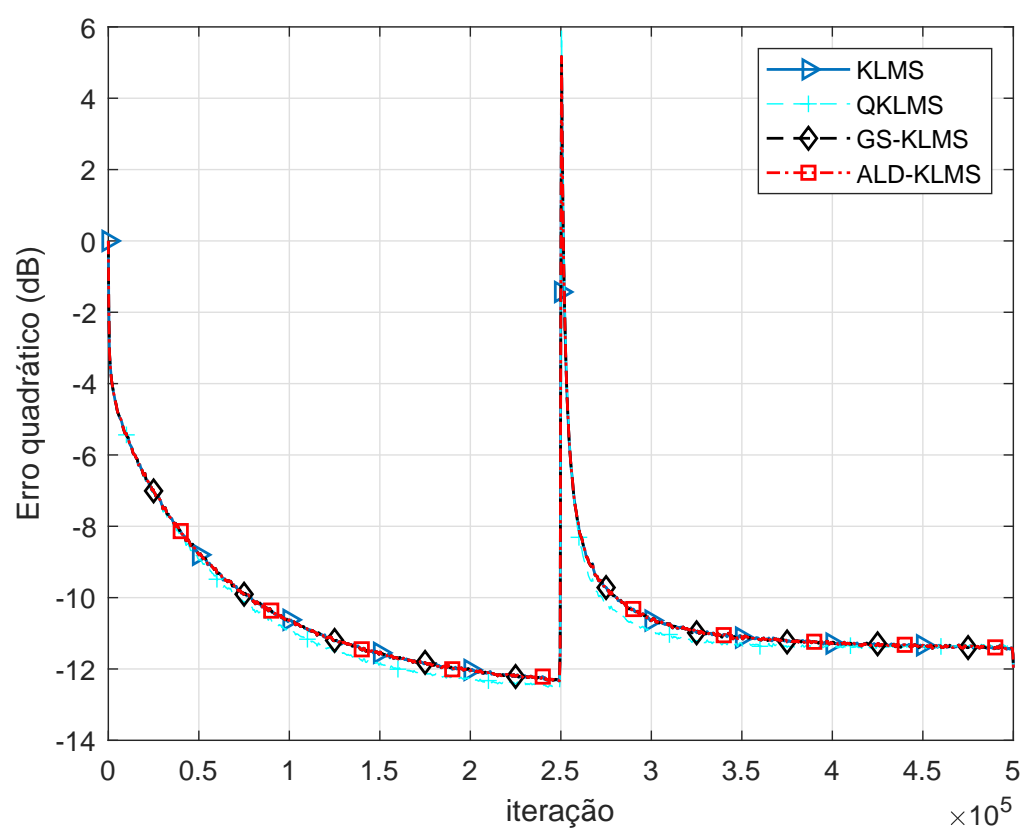

Figura 34: MSE no problema de equalização não linear para kernel polinomial de ordem $q=2$, $\mu=0,0001, M=6, \varepsilon_{Q}=0,75, \varepsilon_{A L D}=\varepsilon_{G S}=0,84$, média de 100 realizações.

Fonte: Autor

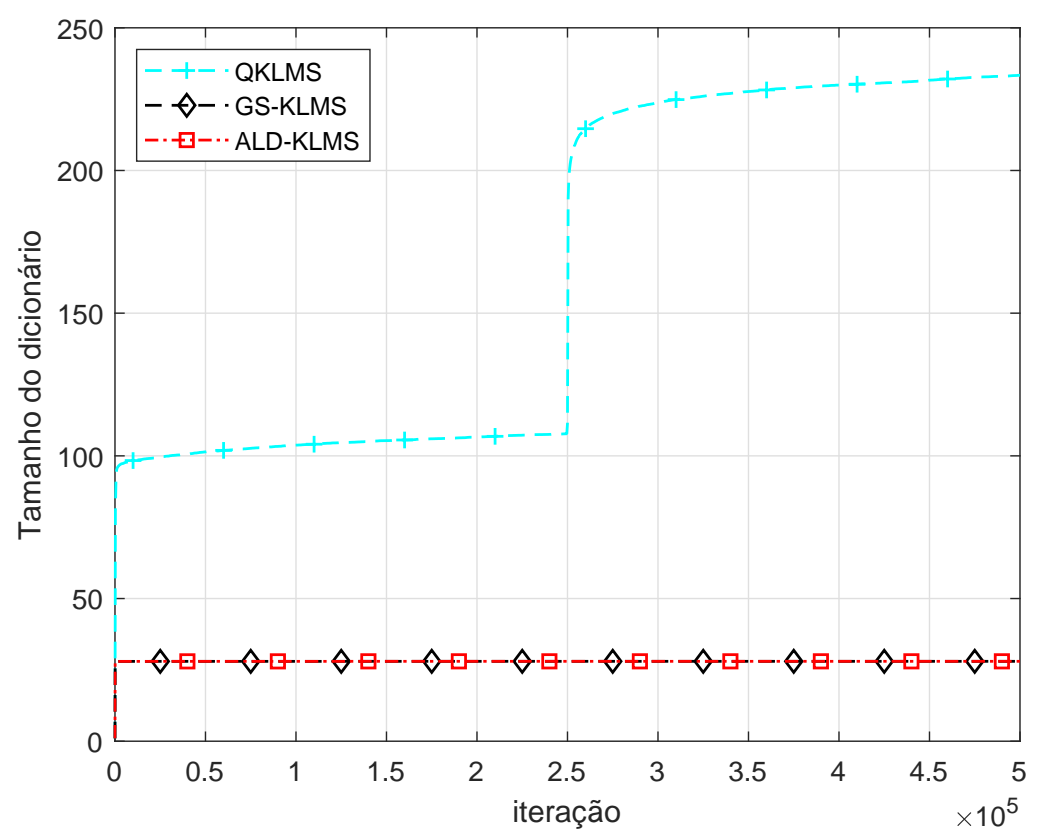

Figura 35: Tamanho do dicionário no problema de equalização não linear para kernel polinomial de ordem $q=2, \mu=0,0001, M=6, \varepsilon_{Q}=0,75, \varepsilon_{A L D}=\varepsilon_{G S}=0,84$ média de 100 realizações.

Fonte: Autor 


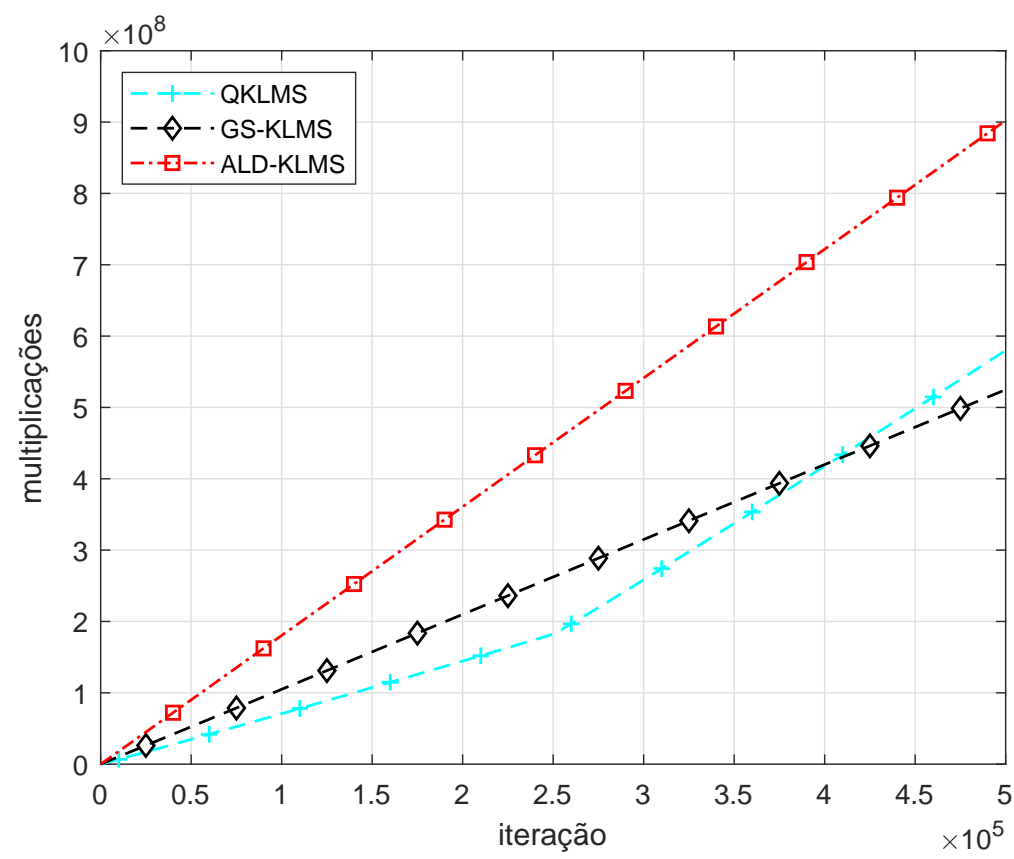

Figura 36: Número de multiplicações acumuladas no problema de equalização não linear para kernel polinomial de ordem $q=2, \mu=0,0001, M=6, \varepsilon_{Q}=0,75, \varepsilon_{A L D}=\varepsilon_{G S}=0,84$ média de 100 realizações.

Fonte: Autor

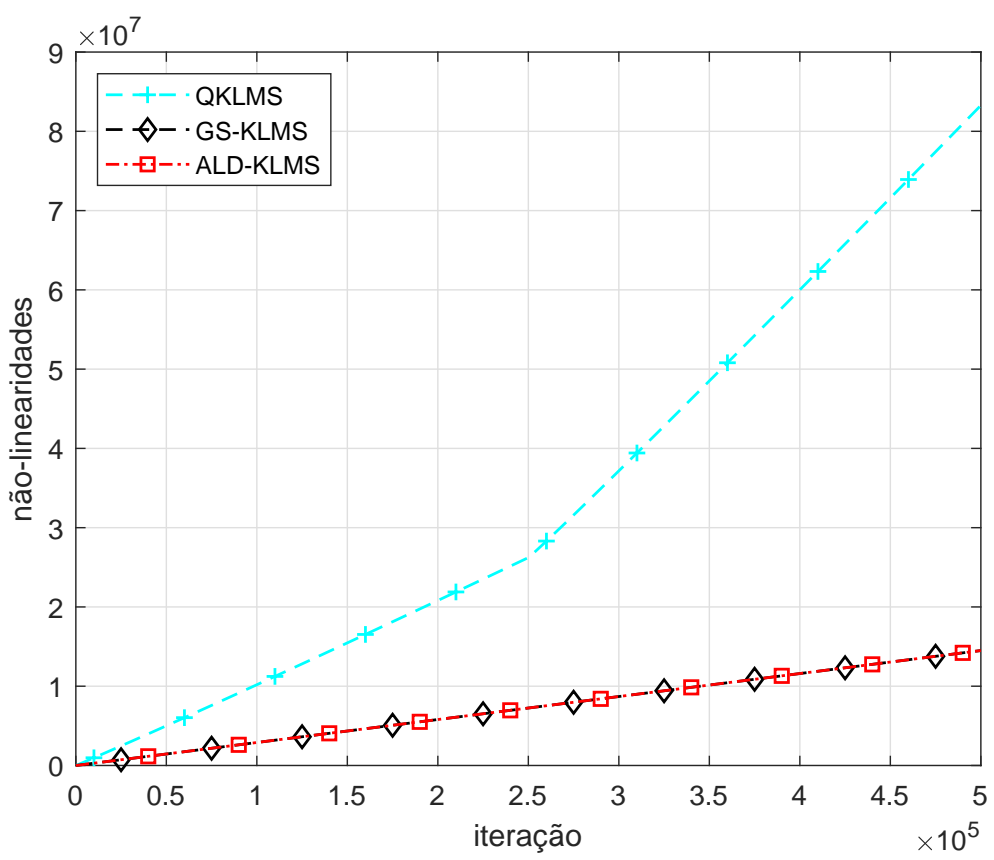

Figura 37: Número de não-linearidades acumuladas no problema de equalização não linear para kernel polinomial de ordem $q=2, \mu=0,0001, M=6, \varepsilon_{Q}=0,75, \varepsilon_{A L D}=\varepsilon_{G S}=0,84$ média de 100 realizações.

Fonte: Autor 


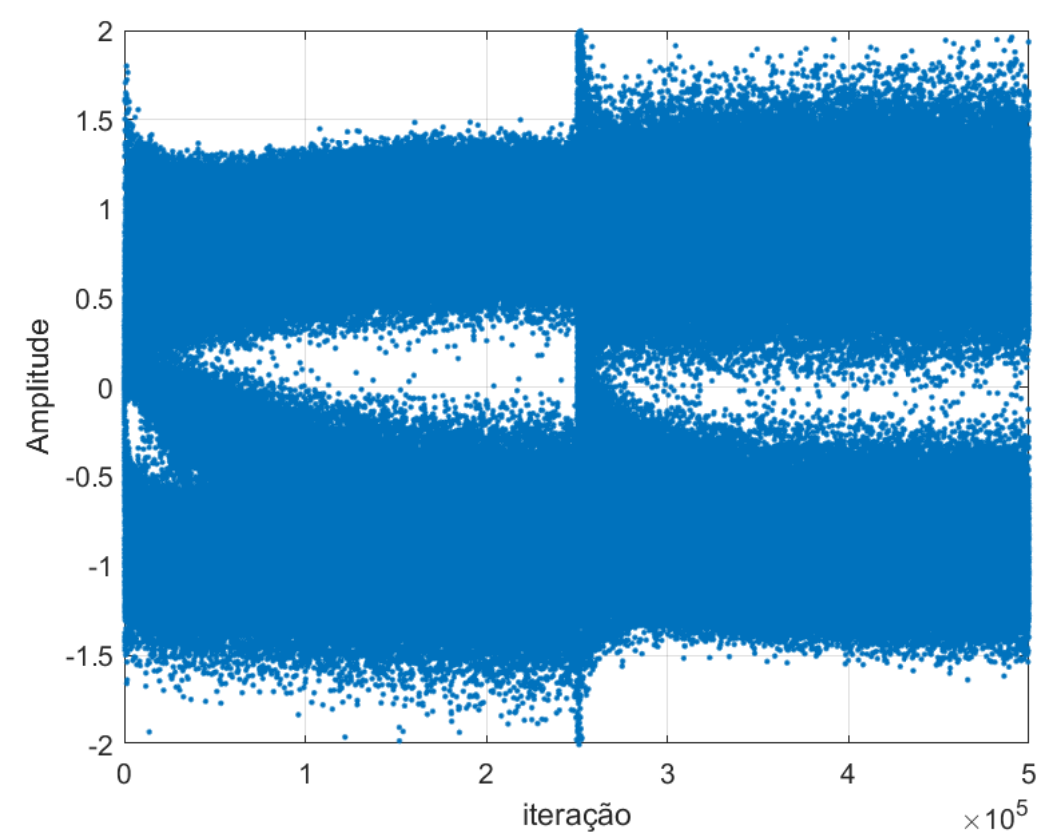

Figura 38: Saída do filtro adaptativo no problema de equalização não linear para kernel polinomial de ordem $q=2, \mu=0,0001, M=6, \varepsilon_{G S}=0,84$.

Fonte: Autor

\subsubsection{Análise do MSE variando-se o $\varepsilon_{G S}$ para kernel gaussiano}

A fim de comparar o desempenho do GS-KLMS para diversos valores de $\varepsilon_{G S}$, mais uma vez se utiliza o problema de predição não linear descrito no Capítulo 1 da página 5. Para esta comparação, os valores de $\varepsilon_{G S}$ usados foram $0,01,0,1,0,5,0,75$ e 0,9 . O kernel utilizado é o gaussiano com parâmetro $\zeta=1$ e $M=7$. O passo de adaptação é $\mu=0,5$. A simulação teve um total de $3 \times 10^{4}$ iterações com a mudança abrupta em $1,5 \times 10^{4}$. É possível verificar pela Figura 39 que há uma variação de até $40 \mathrm{~dB}$ quando comparamos os algoritmos com $\varepsilon_{G S}=0,01$ e $\varepsilon_{G S}=0,9$ na primeira metade do experimento. Na segunda metade essa diferença cai para aproximadamente $25 \mathrm{~dB}$. O número de elementos no dicionário variou entre 161 para $\varepsilon_{G S}=0,01$ e 46 para $\varepsilon_{G S}=0,9$ como se nota na Figura 40. Para esses mesmos valores de $\varepsilon_{G S}$, a diferença em números de operações de multiplicação acumuladas chega a aproximadamente 10 vezes e o número de operações não lineares de mais de 3 vezes, conforme as Figuras 41 e 42 respectivamente. 


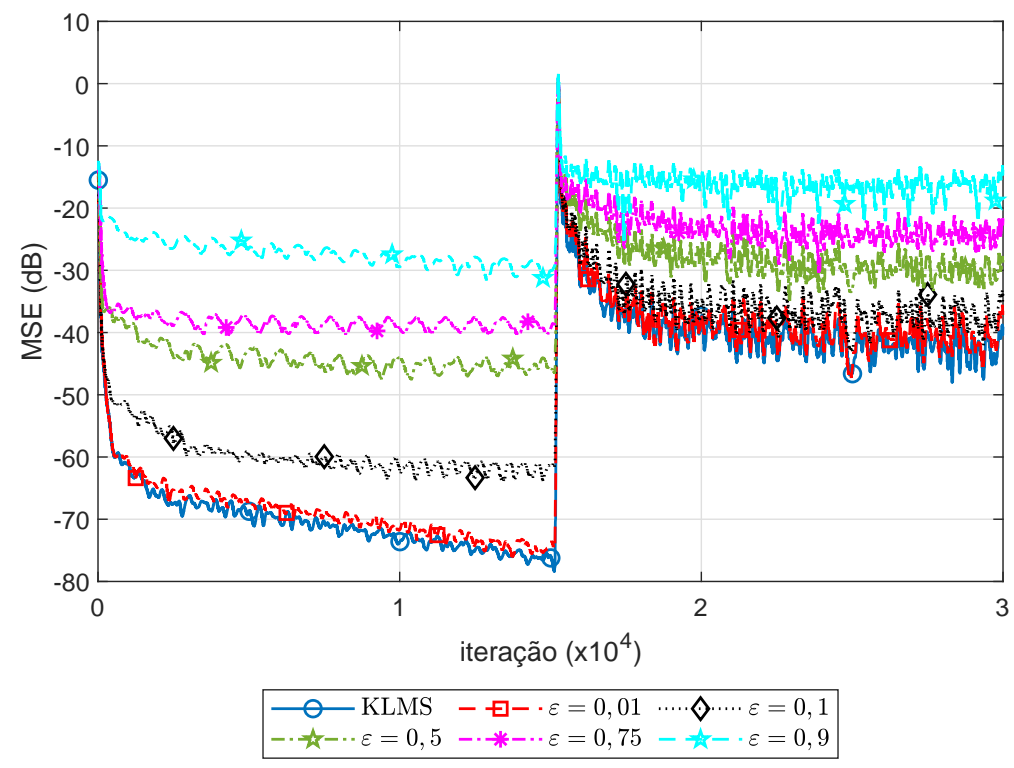

Figura 39: MSE no problema de predição não linear para kernel gaussiano com parâmetro $\zeta=1, \mu=0,5, M=7$, para $\varepsilon_{G S}=0,01, \varepsilon_{G S}=0,1, \varepsilon_{G S}=0,5, \varepsilon_{G S}=0,75$ e $\varepsilon_{G S}=0,9$.

Fonte: Autor

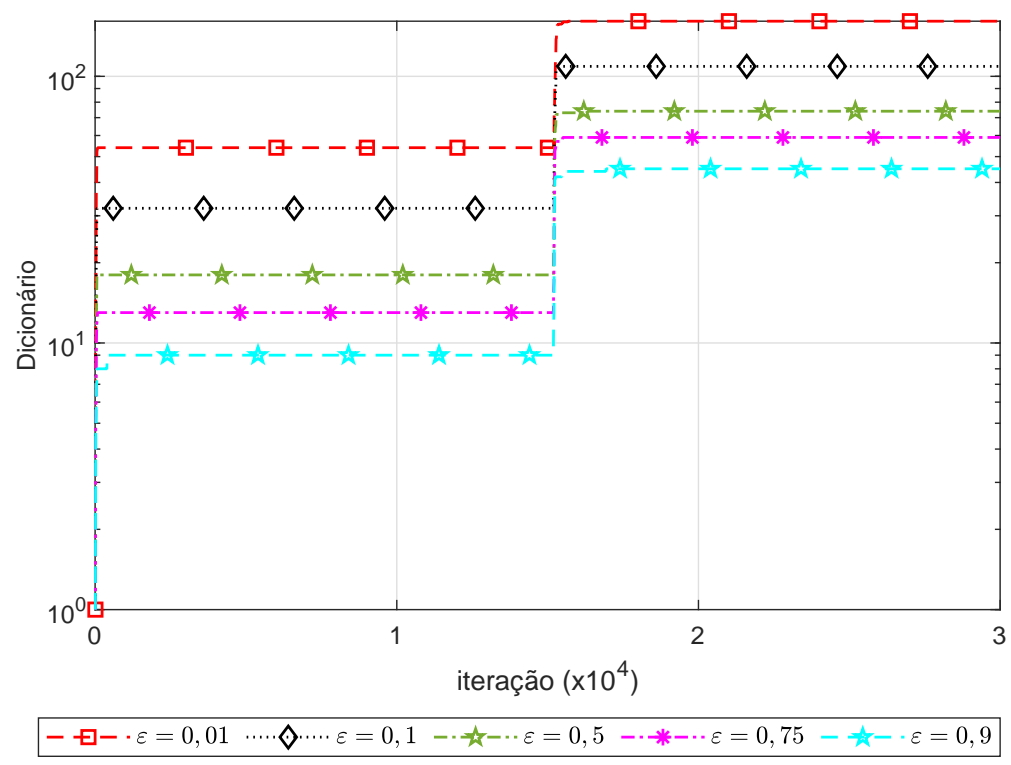

Figura 40: Tamanho do dicionário no problema de predição não linear para kernel gaussiano com parâmetro $\zeta=1, \mu=0,5, M=7$, para $\varepsilon_{G S}=0,01, \varepsilon_{G S}=0,1, \varepsilon_{G S}=0,5, \varepsilon_{G S}=0,75 \mathrm{e}$ $\varepsilon_{G S}=0,9$.

Fonte: Autor 


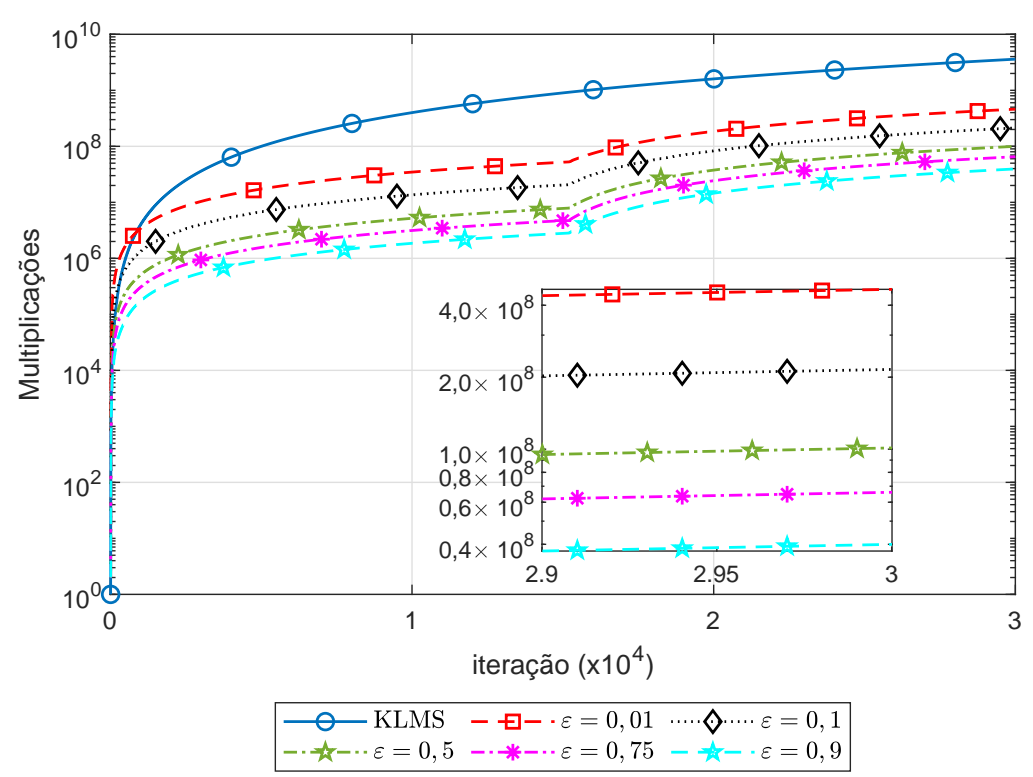

Figura 41: Número de operações de multiplicação no problema de predição não linear para kernel gaussiano com parâmetro $\zeta=1, \mu=0,5, M=7$, para $\varepsilon_{G S}=0,01, \varepsilon_{G S}=0,1, \varepsilon_{G S}=0,5$, $\varepsilon_{G S}=0,75$ e $\varepsilon_{G S}=0,9$.

Fonte: Autor

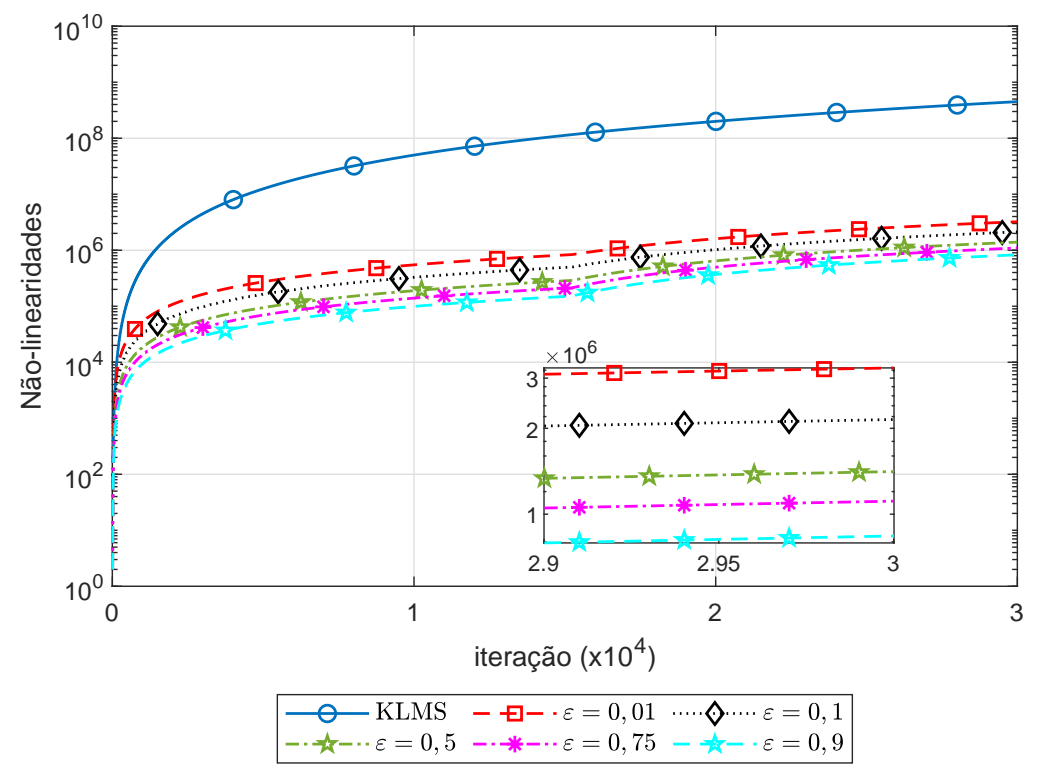

Figura 42: Número de operações não lineares no problema de predição não linear para kernel gaussiano com parâmetro $\zeta=1, \mu=0,5, M=7$, para $\varepsilon_{G S}=0,01, \varepsilon_{G S}=0,1, \varepsilon_{G S}=0,5$, $\varepsilon_{G S}=0,75$ e $\varepsilon_{G S}=0,9$.

Fonte: Autor 


\subsubsection{Análise do MSE variando-se o $\varepsilon_{G S}$ para kernel polinomial}

Para se comparar o desempenho do GS-KLMS com kernel polinomial variando-se o valor de $\varepsilon_{G S}$, recorre-se ao problema de predição não linear descrito no Capítulo 1 da página 5 mais uma vez. Os valores de $\varepsilon_{G S}$ usados foram $0,01,0,1,13,5,15$ e 20 . O kernel utilizado neste experimento é o polinomial de ordem 2 e $M=7$. O passo de adaptação é $\mu=10^{-4}$. A simulação utilizou um total de $3 \times 10^{4}$ iterações com a mudança abrupta em $1,5 \times 10^{4}$. Pode-se perceber na Figura 43 que para valores de $\varepsilon_{G S}$ até 13,5, não há diferenças perceptíveis no desempenho em termos de MSE. Para os valores de $\varepsilon_{G S}=15$ e $\varepsilon_{G S}=20$, há uma demora maior para entrar em regime e não se consegue o mesmo desempenho que os outros na primeira metade do experimento, ficando $7 \mathrm{~dB}$ acima do valor obtido com os outros valores de $\varepsilon_{G S}$. O tamanho dos dicionários obtidos para os diversos valores de $\varepsilon_{G S}$ estão na Figura 44. É possível notar que, ao final do experimento, o dicionário para $\varepsilon_{G S}=0,01$ e $\varepsilon_{G S}=0,1$ tinha 36 elementos e para $\varepsilon_{G S}=$ 20 tinha 20. O número de operações de multiplicação acumuladas e o número de operações não lineares acumuladas estão respectivamente nas Figuras 45 e 46 . É possível verificar que para $\varepsilon_{G S}=20$, o número acumulado de operações de multiplicação é aproximadamente 3 vezes menor que para $\varepsilon_{G S}=0,01$ enquanto que o número de operações não lineares é 2 vezes menor para os mesmo valores de $\varepsilon_{G S}$.

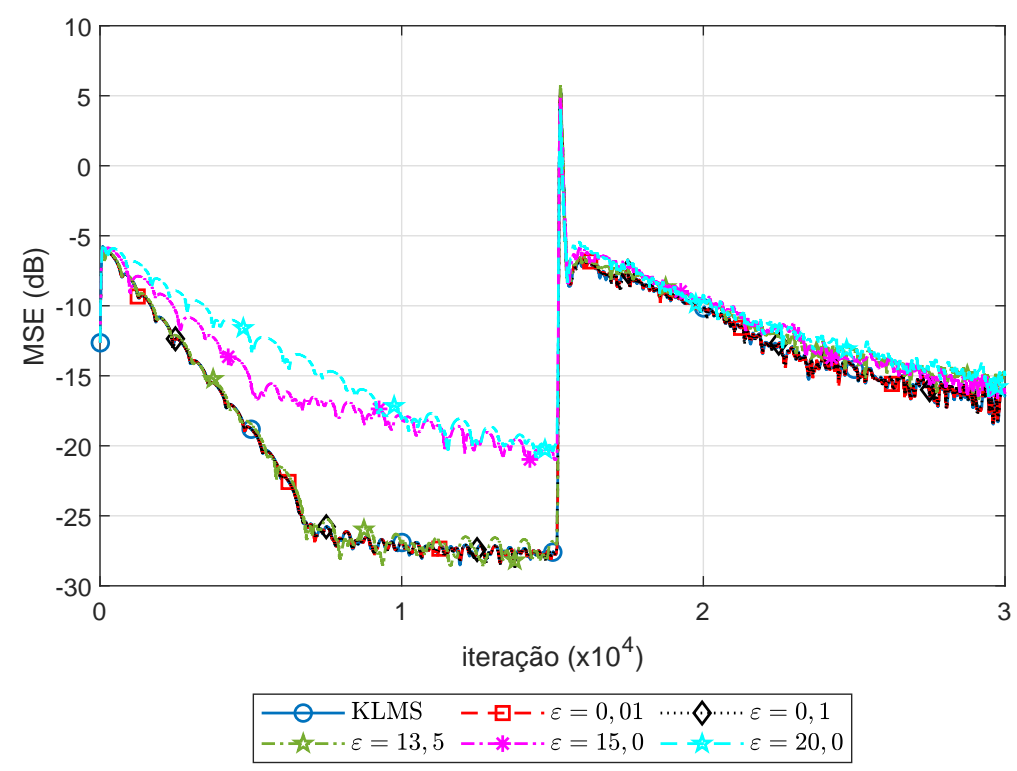

Figura 43: MSE no problema de predição não linear para kernel polinomial de ordem $q=2$, $\mu=0,0001, M=7$, para $\varepsilon_{G S}=0,01, \varepsilon_{G S}=0,1, \varepsilon_{G S}=13,5, \varepsilon_{G S}=15$ e $\varepsilon_{G S}=20$.

Fonte: Autor 


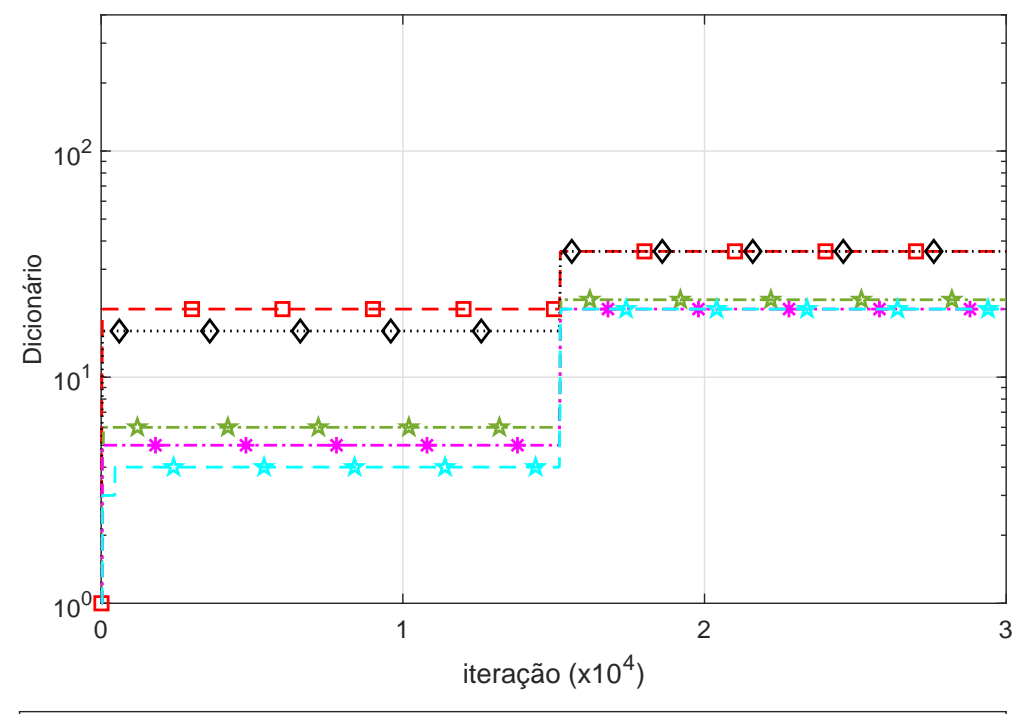

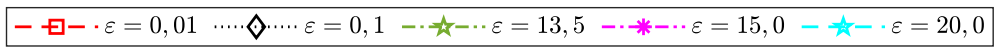

Figura 44: Tamanho do dicionário no problema de predição não linear para kernel polinomial de ordem $q=2, \mu=0,0001, M=7$, para $\varepsilon_{G S}=0,01, \varepsilon_{G S}=0,1, \varepsilon_{G S}=13,5, \varepsilon_{G S}=15 \mathrm{e}$ $\varepsilon_{G S}=20$.

Fonte: Autor

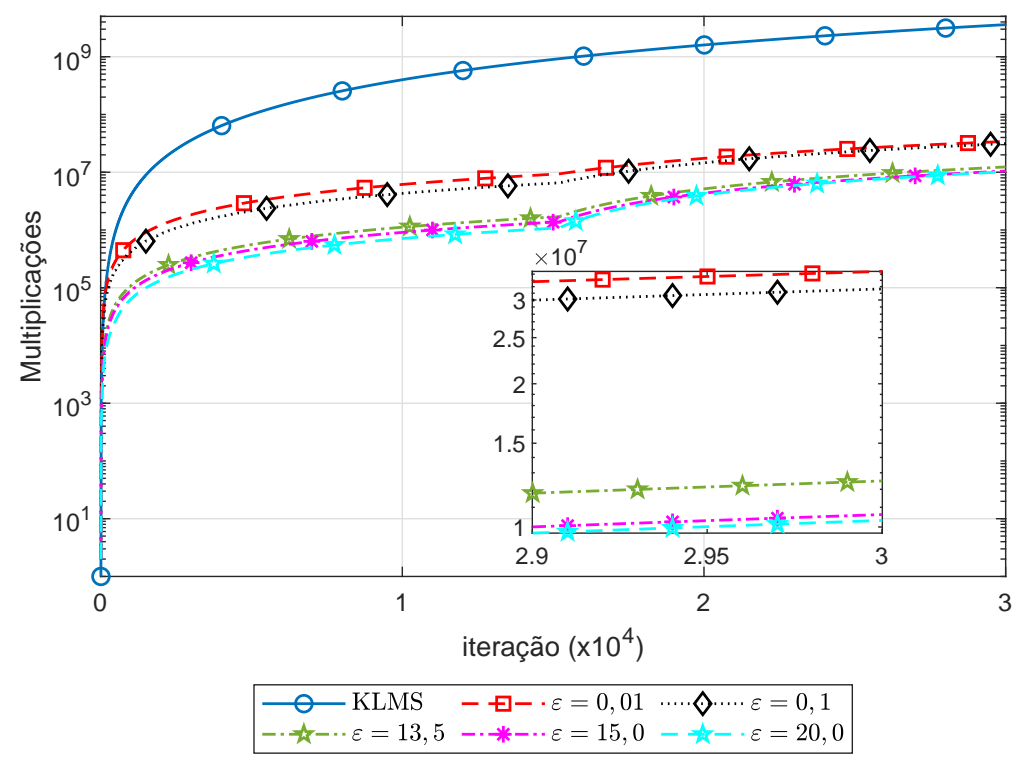

Figura 45: Número de operações de multiplicação no problema de predição não linear para kernel polinomial de ordem $q=2, \mu=0,0001, M=7$, para $\varepsilon_{G S}=0,01, \varepsilon_{G S}=0,1, \varepsilon_{G S}=13,5$, $\varepsilon_{G S}=15$ e $\varepsilon_{G S}=20$.

Fonte: Autor 


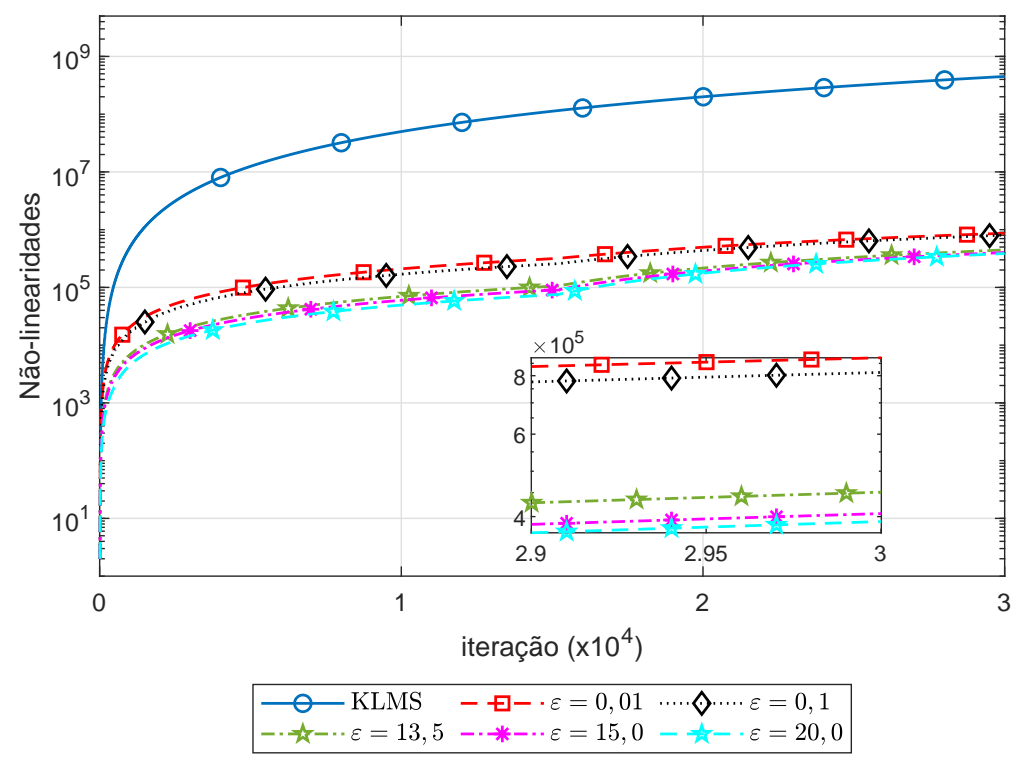

Figura 46: Número de operações não lineares no problema de predição não linear para kernel polinomial de ordem $q=2, \mu=0,0001, M=7$, para $\varepsilon_{G S}=0,01, \varepsilon_{G S}=0,1, \varepsilon_{G S}=13,5$, $\varepsilon_{G S}=15$ e $\varepsilon_{G S}=20$.

Fonte: Autor

Com base nessas simulações e outras que foram feitas em diferentes cenários, conclui-se que a escolha do limiar $\varepsilon_{G S}$ que garante um bom compromisso entre o desempenho em termos de MSE e economia de custo computacional depende da função kernel e do problema específico. Entretanto, independentemente do cenário de simulação e da função kernel, resultados de simulação indicam que é possível escolher um valor do limiar que leva a um bom compromisso e ainda faz do GS-KLMS uma boa alternativa quando comparado com outras soluções da literatura.

\subsection{Retirando elementos do dicionário}

Retirar elementos de um dicionário em um algoritmo como o descrito neste capítulo envolve duas etapas: a primeira é identificar quando se deve retirar um vetor do dicionário e a segunda é alterar a base no espaço $\mathcal{B}$ após a retirada do vetor. Além disso, outro cuidado a ser tomado é a possibilidade de incluir e retirar elementos do dicionário em uma mesma iteração. Para evitar um aumento exagerado do custo, decidiu-se que as duas operações não podem ser realizadas na mesma iteração.

No processo de retirada de elementos, é necessário verificar a influência de cada vetor do 
dicionário no cálculo da saída $y(n)$. Em um algoritmo como o QKLMS, a equação

$$
y(n)=\sum_{i=1}^{N(n)} a_{i} \kappa\left(\mathbf{u}(n), \mathbf{u}_{i}\right)
$$

mostra que a influência de um elemento $\mathbf{u}_{i}$ do dicionário na saída $y(n)$ é dada pela parcela $a_{i} k\left(\mathbf{u}(n), \mathbf{u}_{i}\right)$. Portanto, retira-se o vetor do dicionário que corresponder à menor parcela em relação aos demais. Isso pode ser feito comparando a parcela a um limiar a ser escolhido. Algo análogo é feito, por exemplo, em (WADA; TANAKA, 2017). Diferente de algoritmos que calculam a saída com (4.17), o GS-KLMS calcula uma base a partir dos vetores mapeados e a equação que calcula a saída é dada por

$$
y(n)=\mathbf{w}^{T}(n) \widetilde{\mathbf{p}}(n) .
$$

A seguir, reescreve-se (4.18) na forma (4.17) para propor uma maneira de avaliar a influência de cada elemento do dicionário.

Substituindo (4.8) em (4.18), tem-se

$$
y(n)=\mathbf{w}(n)^{T} \mathbf{H}_{N(n)} \boldsymbol{\kappa}_{N(n)}(\mathbf{u}(n)) .
$$

Definindo o vetor $\mathbf{a}(n) \triangleq\left(\mathbf{w}(n)^{T} \mathbf{H}_{N(n)}\right)^{T}=\left[\begin{array}{llll}a_{1}(n) & a_{2}(n) & \cdots & a_{N(n)}(n)\end{array}\right]^{T}$, pode-se reescrever (4.19) como

$$
y(n)=\mathbf{a}^{T}(n) \boldsymbol{\kappa}_{N(n)}(\mathbf{u}(n))=\sum_{i=1}^{N(n)} a_{i}(n) \kappa\left(\mathbf{u}(n), \mathbf{u}_{i}\right) .
$$

A partir de (4.20), pode-se avaliar a influência de cada elemento do dicionário na saída $y(n)$. Cabe observar que não se deve retirar um elemento do dicionário se ele teve uma baixa influência em apenas uma iteração, pois ele pode acabar sendo incluído novamente logo depois. Para evitar isso, propõe-se considerar a seguinte média temporal

$$
\mathbf{c}(n)=\lambda \mathbf{c}(n-1)+\left|\mathbf{a}(n) \odot \boldsymbol{\kappa}_{N(n)}(\mathbf{u}(n))\right|,
$$

em que $\mathbf{c}(0)=\mathbf{0}, 0<\lambda<1$ é um fator de esquecimento e $\left|\mathbf{a}(n) \odot \boldsymbol{\kappa}_{N(n)}(\mathbf{u}(n))\right|$ é o vetor que contém o valor absoluto de cada componente $a_{i}(n) \kappa\left(\mathbf{u}(n), \mathbf{u}_{i}\right), i=1,2, \ldots N(n)$. Para identificar quais elementos do dicionário devem ser retirados, define-se um limiar $\varepsilon_{R E T}$ que é comparado aos elementos do vetor $\mathbf{c}(n)$. Os elementos que forem menores que o limiar são então retirados do dicionário.

$\mathrm{O}$ algoritmo GS-KLMS atualiza as matrizes $\mathbf{H}_{N(n)}$ e $\mathbf{G}_{N(n)}$ quando um novo vetor é inserido 
no dicionário, pois é assim que a base é implicitamente atualizada. Por isso, quando se retira um elemento do dicionário, a base também precisa ser atualizada e o mesmo acontece com as matrizes $\mathbf{H}_{N(n)}$ e $\mathbf{G}_{N(n)}$. O primeiro passo é remover a linha e coluna do gramiano referentes ao elemento que se quer retirar. O resultado disso será um novo gramiano com os mesmos elementos de antes a menos da linha e coluna que foram retiradas. Para se calcular a nova matriz $\mathbf{H}_{N(n)}$, deve-se usar a decomposição de Cholesky do novo gramiano, definida na Equação (3.25) da página 29. A partir dessa decomposição, obtém-se $\widetilde{\mathbf{P}}^{T}$ cuja inversa é a nova matriz $\mathbf{H}_{N(n)}$. Esses passos estão mostrados na Figura 47 em que as operações necessárias estão identificadas acima das setas entre as matrizes. Deve-se também retirar a linha do vetor $\mathbf{a}(n)$ calculado anteriormente e resolver o sistema linear $\mathbf{H}_{N(n)}^{T} \mathbf{w}(n)=\mathbf{a}(n)$ com a nova $\mathbf{H}_{N(n)}$ e novo $\mathbf{a}(n)$ para assim obter o novo vetor $\mathbf{w}(n)$.

A complexidade computacional desse algoritmo é alta, já que só a decomposição de Cholesky tem custo $O\left(N^{3}\right)$. Portanto, caso se necessite retirar elementos repetidas vezes do dicionário, esse algoritmo pode não ser muito atrativo em problemas que exigem soluções em tempo real. Porém, quando a necessidade de retirar elementos do dicionário é baixa, este algoritmo se mostra útil. O algoritmo GS-KLMS considerando-se a retirada de elementos do dicionário pode ser visto na Tabela 8. Para evitar que se retire elementos do dicionário a cada iteração, foi considerado um contador com um limite $\varepsilon_{C T R}$. $\mathrm{O}$ algoritmo só realiza as operações necessárias para a retirada dos elementos caso o contador ultrapasse o número de iterações definidas por $\varepsilon_{C T R}$. Esse contador só é zerado quando se retira um elemento do dicionário.

\subsubsection{Resultados de Simulação}

Considerando novamente o exemplo do Capítulo 1 da página 5, o algoritmo GS-KLMS retirando elementos do dicionário, denotado por GS-KLMS-RM, é comparado com o algoritmo KLMS sem esparsificação e com o GS-KLMS. Considerou-se um fator de esquecimento de $\lambda=$ 0,999 e para o limiar $\varepsilon_{R E T}=10^{-5}$. Três valores para o parâmetro $\varepsilon_{C T R}$ foram considerados: 1 , 10 e 100. Os outros parâmetros permanecem os mesmos. A Figura 48 mostra o erro quadrático médio do GS-KLMS-RM comparado ao GS-KLMS sem retirar elementos e ao KLMS sem esparsificação. É possível notar uma uma pequena degradação no desempenho. Já a Figura 49 mostra o o tamanho do dicionário das duas versões do GS-KLMS. Verifica-se que o algoritmo $\operatorname{com} \varepsilon_{C T R}=1$ tem na segunda metade da simulação uma média de 114 elementos no dicionário, enquanto para $\varepsilon_{C T R}=10$ esse número é de 165 e para $\varepsilon_{C T R}=100,173$. O GS-KLMS tem um 
G original

$\left(\begin{array}{ccccc}g_{11} & g_{12} & g_{13} & \cdots & g_{1 N} \\ g_{21} & g_{22} & g_{23} & \cdots & g_{2 N} \\ g_{31} & g_{32} & g_{33} & \cdots & g_{3 N} \\ \vdots & \vdots & \vdots & \ddots & \vdots \\ g_{N 1} & g_{N 2} & g_{N 3} & \cdots & g_{N N}\end{array}\right) \quad$ Retira $\quad\left(\begin{array}{cccc}g_{11} & g_{13} & \cdots & g_{1 N} \\ g_{31} & g_{33} & \cdots & g_{3 N} \\ \vdots & \vdots & \ddots & \vdots \\ g_{N 1} & g_{N 3} & \cdots & g_{N N}\end{array}\right)$

Cholesky

$\left(\begin{array}{cccc}h_{11} & 0 & \cdots & 0 \\ h_{21} & h_{22} & \cdots & 0 \\ \vdots & \vdots & \ddots & \vdots \\ h_{(N-1) 1} & h_{(N-1) 2} & \cdots & h_{(N-1)(N-1)}\end{array}\right)$ Inverte $\left.\quad \begin{array}{cccc}p_{11} & 0 & \cdots & 0 \\ p_{12} & p_{22} & \cdots & 0 \\ \vdots & \vdots & \ddots & \vdots \\ p_{1(N-1)} & p_{2(N-1)} & \cdots & p_{(N-1)(N-1)}\end{array}\right)$

H modificada

$\widetilde{\mathbf{P}}^{T}$

Figura 47: Obtenção das novas matrizes $\mathbf{G}$ e $\mathbf{H}$

Fonte: Autor.

dicionário de 177 elementos ao final da simulação. O número de multiplicações acumuladas em $n=10^{4}$ do GS-KLMS é de 9,6 $\times 10^{7}$, enquanto que o GS-KLMS-RM com $\varepsilon_{C T R}=1$, $\varepsilon_{C T R}=10$ e $\varepsilon_{C T R}=100$ realizam respectivamente $5,3 \times 10^{8}, 4,6 \times 10^{8}$ e $1,7 \times 10^{8}$. Já o número de operações não lineares acumuladas em $n=10^{4}$ realizadas pelo GS-KLMS foi de $1,1 \times 10^{6}$, já o GS-KLMS-RM com $\varepsilon_{C T R}=1, \varepsilon=10$ e $\varepsilon=100$ realiza respectivamente $1,2 \times 10^{8}, 9,7 \times 10^{7}$ e $2,0 \times 10^{6}$. Essa simulação mostra que apesar de se conseguir diminuir o número de elementos do dicionário, o algoritmo proposto não é vantajoso em termos de custo computacional.

\subsection{Conclusão}

O algoritmo GS-KLMS, que utiliza a técnica de esparsificação apresentada no Capítulo 3, foi apresentado. Seu desempenho foi comparado com algoritmos KLMS que utilizam as técnicas de esparsificação apresentadas no Capítulo 2. Pode-se perceber, a partir de cenários de simulação diferentes, que o GS-KLMS atinge o mesmo desempenho em termos de MSE dos outros algoritmos, realizando menos multiplicações, o que o torna vantajoso. Em termos de operações não lineares, o GS-KLMS realiza o mesmo número de operações que o ALD-KLMS, sendo este número menor que o dos outros algoritmos. Como o valor do limiar $\varepsilon_{G S}$ afeta di- 


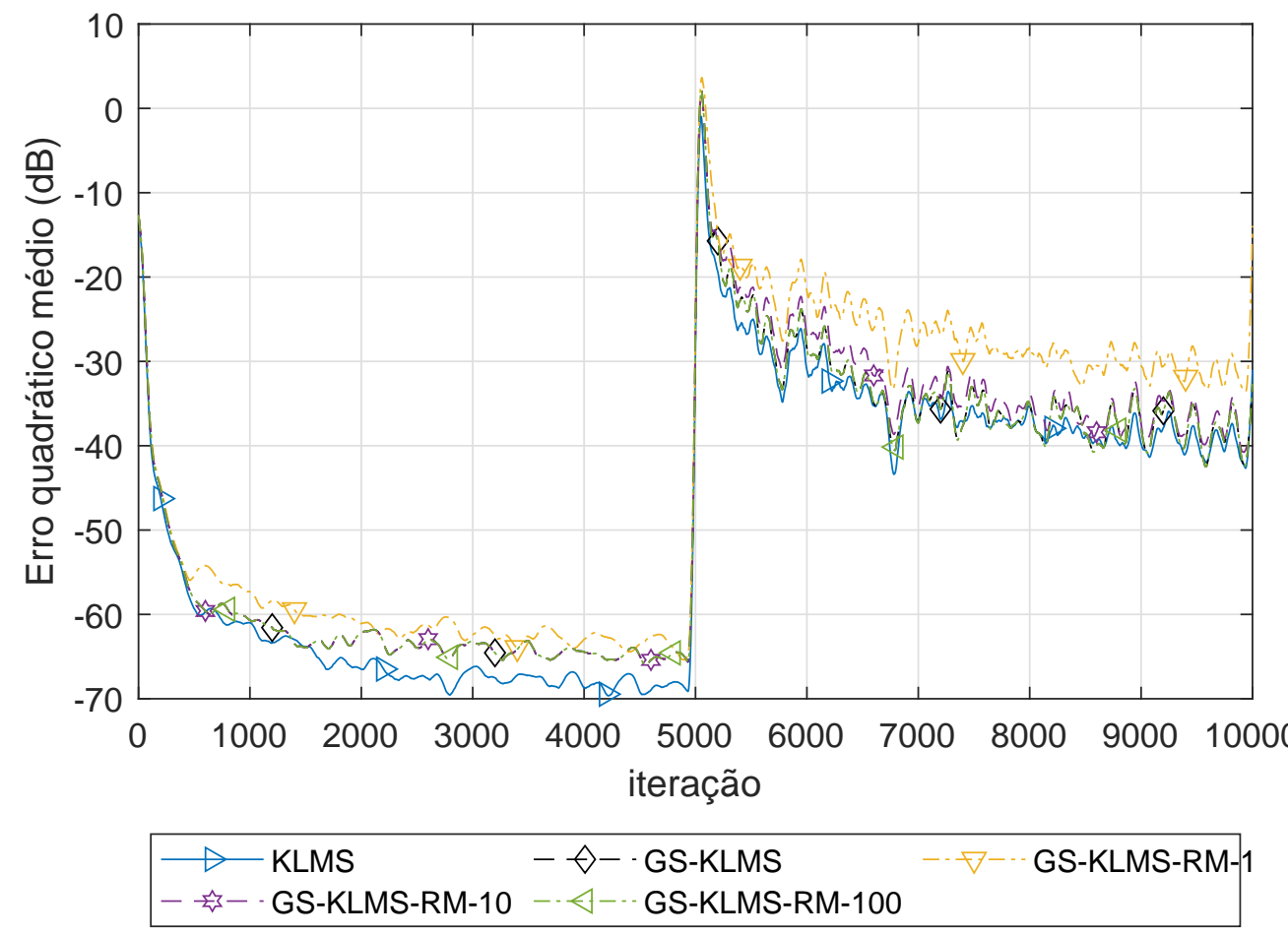

Figura 48: MSE para o problema de predição não linear considerando kernel gaussiano, $\zeta=1$, $M=7, \mu=0,5, \varepsilon_{G S}=0,0199, \lambda=0,999, \varepsilon_{R E T}=10^{-5}, \varepsilon_{C T R 1}=1, \varepsilon_{C T R 2}=10$ e $\varepsilon_{C T R 3}=100$.

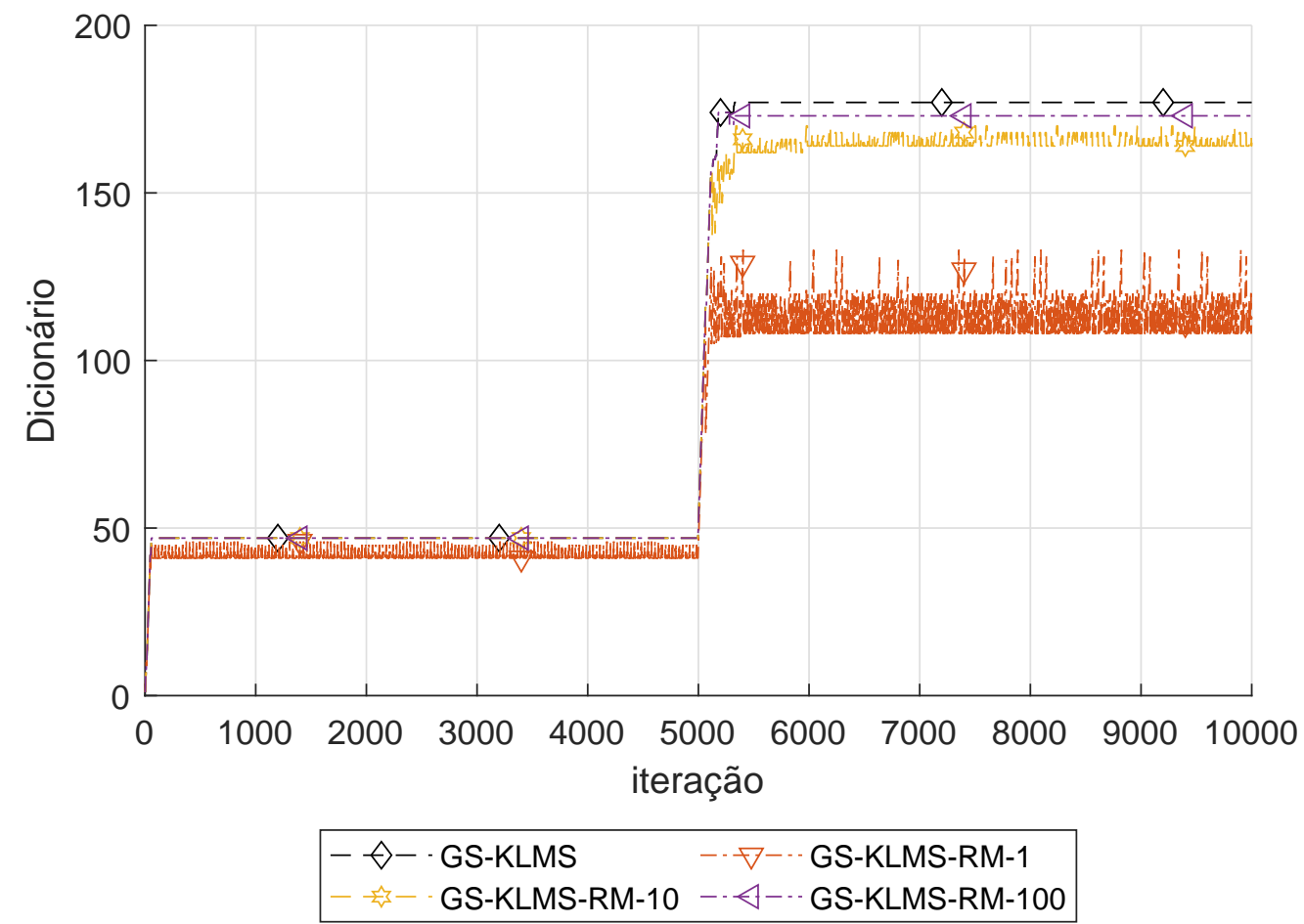

Figura 49: Tamanho do dicionário para o problema de predição não linear considerando kernel gaussiano, $\zeta=1, M=7, \mu=0,5, \varepsilon_{G S}=0,0199, \lambda=0,999, \varepsilon_{R E T}=10^{-5}, \varepsilon_{C T R 1}=1, \varepsilon_{C T R 2}=10$ e $\varepsilon_{C T R 3}=100$. 


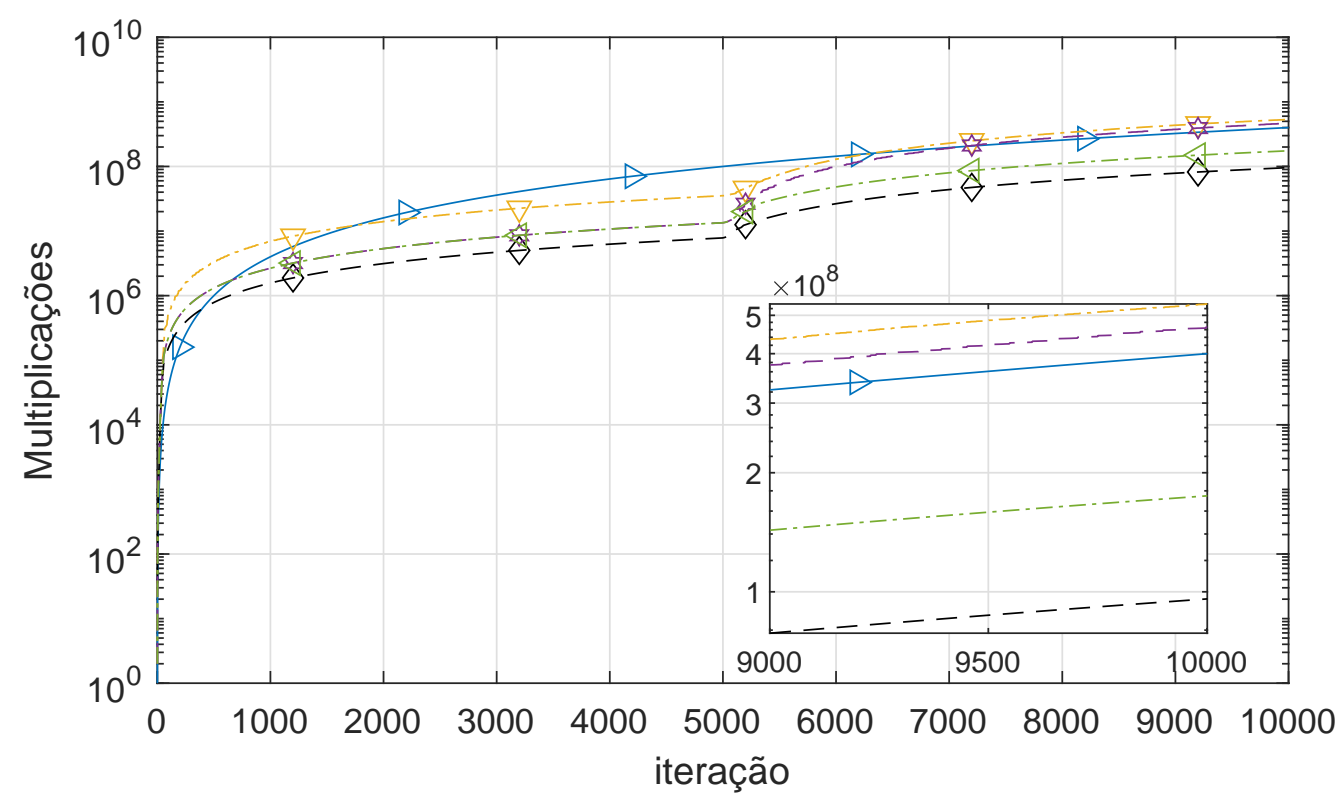

\begin{tabular}{|lll|}
\hline$\triangleright$ & KLMS & $-\diamond-\cdot$ GS-KLMS \\
$-\nabla-\cdots$ GS-KLMS-RM-1 & $-\downarrow-$ GS-KLMS-RM-10 \\
$-\triangleleft-\cdots$ GS-KLMS-RM-100 & \\
\hline
\end{tabular}

Figura 50: Número acumulado de operações de multiplicações para o problema de predição não linear considerando kernel gaussiano, $\zeta=1, M=7, \mu=0,5, \varepsilon_{G S}=0,0199, \lambda=0,999$, $\varepsilon_{R E T}=10^{-5}, \varepsilon_{C T R 1}=1, \varepsilon_{C T R 2}=10$ e $\varepsilon_{C T R 3}=100$.

Fonte: Autor

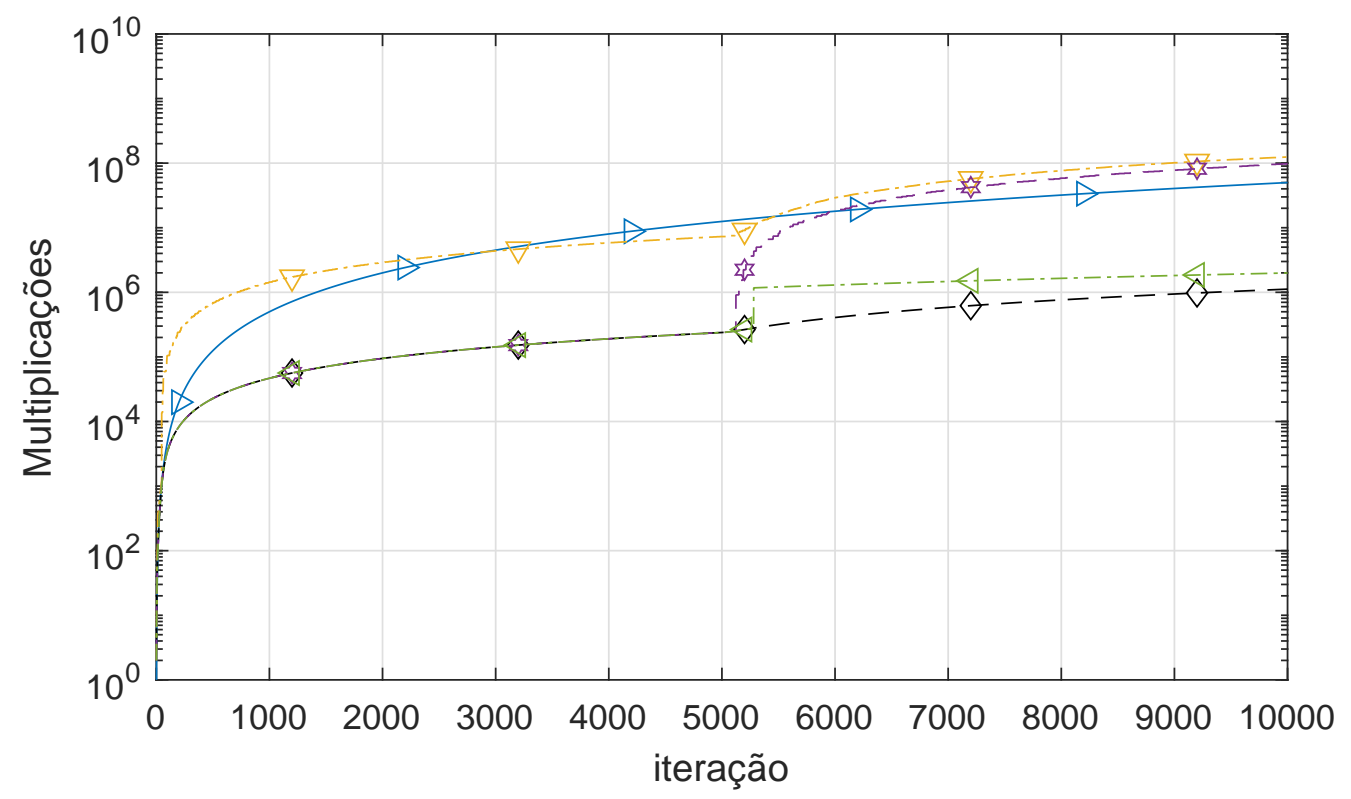

\begin{tabular}{|ll|}
\hline$\triangleright$ KLMS & $-\diamond-\cdot$ GS-KLMS \\
$-\nabla-\cdot$ GS-KLMS-RM-1 & $-\downarrow-\cdot$ GS-KLMS-RM-10 \\
$-\cdot \triangleleft-\cdots$ GS-KLMS-RM-100 & \\
\hline
\end{tabular}

Figura 51: Número acumulado de operações não lineares para o problema de predição não linear considerando kernel gaussiano, $\zeta=1, M=7, \mu=0,5, \varepsilon_{G S}=0,0199, \lambda=0,999$, $\varepsilon_{R E T}=10^{-5}, \varepsilon_{C T R 1}=1, \varepsilon_{C T R 2}=10$ e $\varepsilon_{C T R 3}=100$. 
retamente a cardinalidade do dicionário, ele deve ser escolhido com cuidado para evitar uma degradação de desempenho elevada e ao mesmo tempo garantir economia de custo computacional. Embora o melhor valor de $\varepsilon_{G S}$ dependa da função kernel e do cenário de simulação, observou-se por meio de simulações que é possível escolher $\varepsilon_{G S}$ que leva a um bom compromisso entre desempenho em termos de MSE e custo computacional e ainda faz do GS-KLMS uma boa alternativa quando comparado a outras soluções da literatura. Uma forma de retirar elementos do dicionário também foi proposta e uma simulação usando o mesmo problema apresentado no Capítulo 1 foi apresentada. Ela mostra que é possível manter praticamente o mesmo desempenho em termos de erro quadrático com menos elementos no dicionário. No entanto, ainda é necessário buscar uma alternativa menos custosa para torna-la vantajosa em termos de custo computacional. 
Tabela 8: Sumário do algoritmo GS-KLMS removendo elementos do dicionário

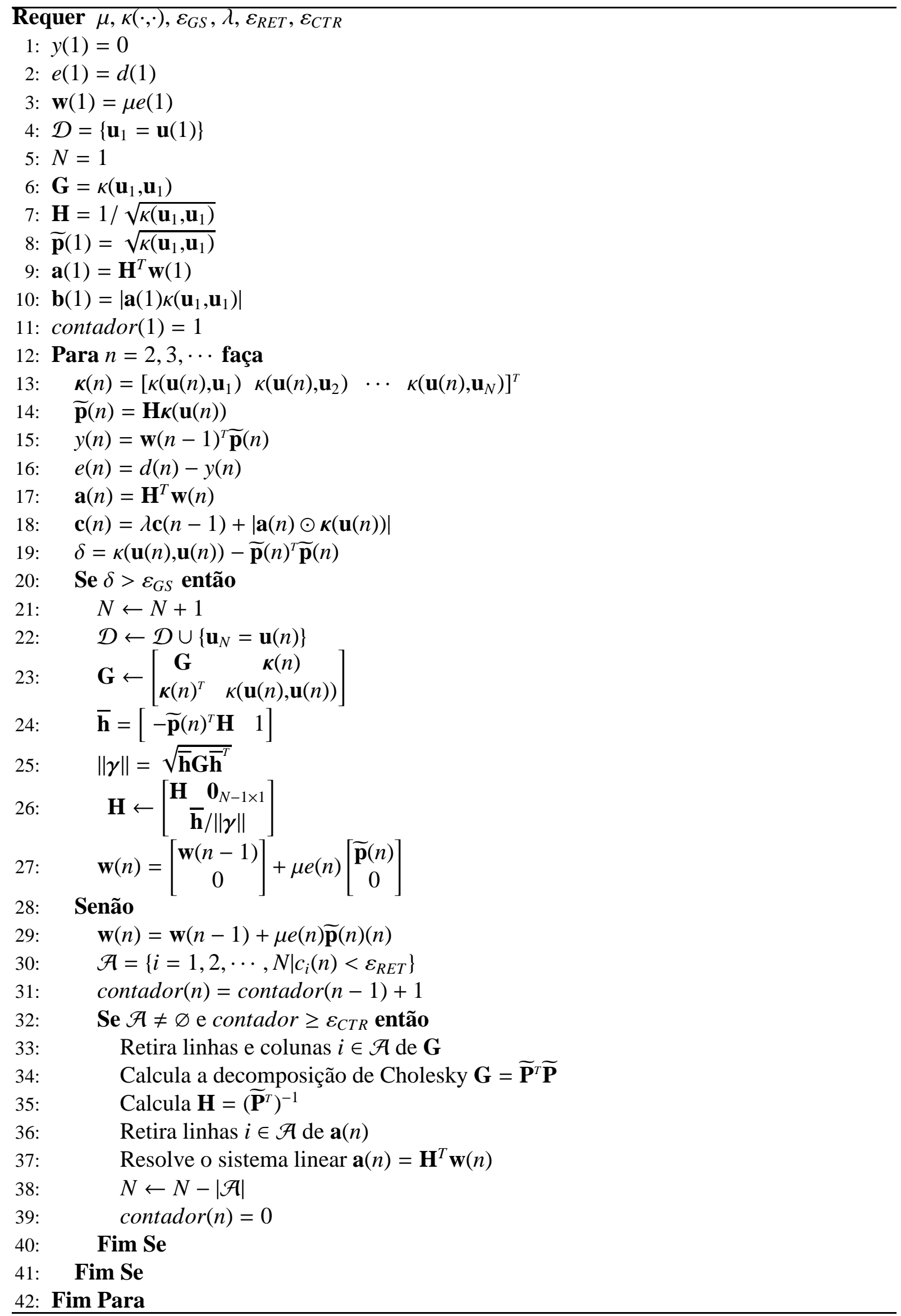




\section{CONCLUSÕES}

Problemas não lineares permeiam a engenharia como, por exemplo, a identificação de sistemas, a predição de séries temporais, a equalização de canais de comunicação, a classificação, a regressão, entre outros. Em muitos desses problemas, filtros adaptativos baseados em kernel têm se destacado na literatura. Porém, na forma como foram originalmente definidos, esses filtros necessitam armazenar e utilizar nos cálculos todos os valores de entrada usados até o instante em questão, armazenados em um dicionário. Com isso, a necessidade de memória e de processamento aumentam linearmente com o tempo. Para que não haja esse crescimento linear, métodos de esparsificação de dicionários foram criados.

Apesar da literatura conter uma grande variedade de técnicas de esparsificação, como as revisitadas no Capítulo 2, a busca por novas técnicas que reduzam ainda mais o custo sem degradar muito o desempenho é de interesse. Neste contexto, foi proposta neste trabalho uma técnica de esparsificação baseada em projeções ortogonais e em um espaço de dimensão finita cuja base é ortonormal. Para a obtenção dessa base, uma forma matricial do processo de Gram-Schimidt é utilizada. Os vetores a serem inseridos no dicionário, cujos vetores mapeados são usados para formar a base mencionada, são selecionados a partir da norma da componente ortogonal ao espaço onde estão sendo projetados. Com isso, é possível verificar que as aproximações dos produtos internos dos vetores mapeados calculadas usando as projeções têm um erro limitado.

A aplicação da técnica proposta ao algoritmo KLMS levou ao algoritmo GS-KLMS, cujo desempenho foi comparado ao de outros algoritmos do tipo KLMS. Resultados de simulação mostraram que o GS-KLMS realiza menos operações de multiplicação e operações não lineares que os outros. Uma proposta para se retirar elementos do dicionário é apresentada. Apesar de se verificar que é possível manter um desempenho comparável retirando elementos do dicionário, a técnica proposta apresenta um custo computacional que não a torna atrativa e ainda merece uma maior investigação.

Como propostas de trabalhos futuros, pode-se considerar:

- Análise do comportamento estocástico do GS-KLMS. Para isso, pretende-se se inspirar nas análises feitas em (CHEN et al., 2012b; PARREIRA et al., 2012); 
- Utilização de um dicionário previamente estabelecido, o que manteria constante o custo computacional de incrementar as matrizes $\mathbf{G}$ e H. Com isso, seria possível criar versões distribuídas do algoritmo GS-KLMS já que um dicionário comum entre os diversos nós já estaria estabelecido;

- Utilização da técnica proposta em problemas de aprendizagem de máquina como no Kernel Principal Component Analysis (KPCA) onde as projeções poderiam tomar o lugar dos vetores mapeados e, com isso, realizar um PCA convencional onde se espera que a matriz de covariância resultante tenha dimensões menores que o gramiano dos valores das amostras.

- Em máquinas de vetores de suporte (SVM), seria possível criar uma base a partir dos vetores de suporte e usar a projeção desses para se obter os pesos, diminuindo o número de operações necessárias na avaliação de uma nova entrada;

- Buscar melhorias para a técnica de retirada de elementos do dicionário. Para tomar a decisão se o vetor deve ser retirado ou não, pretende-se usar a medida de coerência do dicionário;

- Construção prévia do dicionário levando em consideração a distribuição estatística do sinal de entrada e comparação com outras técnicas de esparsificação em ambientes estacionários;

- Quando ocorre uma variação abrupta no ambiente, pretende-se comparar o procedimento de tirar elementos do dicionário com uma nova construção do dicionário em termos de custo computacional;

- Simular o algoritmo proposto em ambientes não estacionários, pois nesses casos há indícios de que o ganho em termos de custo computacional pode ser muito maior;

- Comparar os algoritmos em diferentes ambientes com ruído. 


\section{REFERÊNCIAS}

ALLIGOOD, K. T.; SAUER, T.; YORKE, J. A. Chaos: An Introduction to Dynamical Systems. New York: Springer-Verlag, 1997. (Textbooks in Mathematical Sciences).

BISHOP, C. M. Pattern Recognition and Machine Learning. [S.1.]: Springer Science+Business Media, LLC, 2006.

BOUBOULIS, P.; POUGKAKIOTIS, S.; THEODORIDIS, S. Efficient klms and krls algorithms: A random fourier feature perspective. In: IEEE STATISTICAL SIGNAL PROCESSING WORKSHOP (SSP)Proceedings.... [S.1.: s.n.], 2016. p. 1-5.

CHEN, B.; ZHAO, S.; ZHU, P.; PRÍNCIPE, J. Quantized kernel least mean square algorithm. IEEE Transactions on Neural Networks and Learning Systems, v. 23, n. 1, p. 22-32, Jan 2012. ISSN 2162-237X.

CHEN, B.; ZHAO, S.; ZHU, P.; PRÍNCIPE, J. Mean square convergence analysis for kernel least mean square algorithm. Signal Processing, v. 92, n. 11, p. 2624-2632, Nov 2012.

COMMINIELLO, D.; PRINCIPE, J. C. (Ed.). Adaptive learning methods for nonlinear system modeling. Oxford: Elsevier, 2018.

ENGEL, Y.; MANNOR, S.; MEIR, R. The kernel recursive least-squares algorithm. IEEE Transactions on Signal Processing, v. 52, n. 8, p. 2275-2285, Aug. 2004. ISSN 1053-587X.

GOODFELlOW, I.; BENGIO, Y.; COURVILLE, A. Deep Learning. [S.1.]: MIT Press, 2016.

HATHAWAY, D. The sunspot cycle. Mar. 2017.

Disponível em: <http://solarscience.msfc.nasa.gov/SunspotCycle.shtml>.

LATHI, B. Modern Digital and Analog Communication Systems. [S.1.]: Oxford University Press, Inc., 1998.

LIU, W.; POKHAREL, P. P.; PRINCIPE, J. C. The kernel least-mean-square algorithm. IEEE Transactions on Signal Processing, v. 56, n. 2, p. 543-554, Feb 2008. ISSN 1053-587X.

LIU, W.; PRÍNCIPE, J. C.; HAYKIN, S. Kernel Adaptive Filtering: A Comprehensive Introduction. [S.1.]: Wiley, 2010.

MEYER, C. D. Matrix analysis and applied linear algebra. [S.1.]: Siam, 2000. v. 2.

MURPHY, K. P. Machine Learning: A Probabilistic Perspective. [S.l.: s.n.], 2012.

PARREIRA, W. D.; BERMUDEZ, J. C. M; RICHARD, C.; TOURNERET, J. Y. Stochastic Behavior Analysis of the Gaussian Kernel Least-Mean-Square Algorithm.

IEEE Transactions on Signal Processing, v. 60, n. 5, p. 2208-2222,

Maio 2012. ISSN 1053-587X. 
RAHIMI, A.; RECHT, B. Random features for large-scale kernel machines. In: PLATT, J. C. et al. (Ed.). Advances in Neural Information Processing Systems 20. Curran Associates, Inc., 2008. p. 1177-1184.

RICHARD, C.; BERMUDEZ, J. C. M.; HONEINE, P. Online prediction of time series data with kernels. IEEE Transactions on Signal Processing, v. 57, n. 3, p. 1058-1067, Mar. 2009. ISSN 1053-587X.

SAIDÉ,C.; LENGELLÉ,R.; HONEINE,P.; RICHARD,C.; ACHKAR,R. Nonlinear adaptive filtering using kernel-based algorithms with dictionary adaptation. In: Int. J. Adapt. Control Signal Process v. 29, n. 11, p. 1391-1410, Mar. 2015.

SCHOLKOPF, B.; SMOLA, A. J. Learning with Kernels: support vector machines, regularization, optimization, and beyond. [S.1.]: MIT Press, Cambridge, 2002.

SILVA, M. T. M.; CANDIDO, R.; J. ARENAS-GARCÍA, J.; AZPICUETA-RUIZ.,J. A. Improving multikernel adaptive filtering with selective bias. In: IEEE INT. CONF. ACOUSTICS, SPEECH, AND SIGNAL PROCESS.Proceedings... Calgary, Canada: [s.n.], 2018. p. 4529-4533.

SINGH, A.; AHUJA, N.; MOULIN, P. Online learning with kernels: Overcoming the growing sum problem. In: IEEE INTERNATIONAL WORKSHOP ON MACHINE LEARNING FOR SIGNAL PROCESSING Proceedings.... [S.1.: s.n.], 2012. p. 1-6.

TEAMGRIZZLY. Performing nonlinear classification via linear separation in higher dimensional space. 2010.

Disponível em: <https://www.youtube.com/watch?v=9NrALgHFwTo>.

VAPNIK, V. N. The nature of statistical learning theory. [S.1.]: Springer-Verlag New York, Inc., 1995.

WADA, T.; TANAKA, T. Doubly adaptive kernel filtering. In: ASIA-PACIFIC SIGNAL AND INFORMATION PROCESSING ASSOCIATION ANNUAL SUMMIT AND CONFERENCE (APSIPA ASC)Proceedings.... [S.1.: s.n.], 2017. p. 904-909.

YUKAWA, M. Multikernel adaptive filtering. IEEE Transactions on Signal Processing, v. 60, n. 9, p. 4672-4682, Sep. 2012. ISSN 1053-587X. 